\title{
Theory of hole propagation in one dimensional insulators and superconductors
}

\author{
S. Sorella \\ Istituto Nazionale di Fisica della Materia and \\ International School for Advanced Studies, Via Beirut 4, 34013 Trieste, Italy \\ A. Parola \\ Istituto Nazionale di Fisica della Materia and \\ Istituto di Scienze Fisiche, Universitá di Milano, Via Lucini 3, Como, Italy.
}

\begin{abstract}
The dynamical properties of hole motion in an antiferromagnetic background are determined in one dimensional models in zero magnetic field, where spin isotropy holds, as well as in an external magnetic field. The latter case is also relevant, via particle-hole transformation, to the problem of hole propagation in one dimensional "superconductors". The singularities in the spectral function are investigated by means of bosonization techniques and perturbation theories. Results are then compared with Bethe ansatz solutions and Lanczos diagonalizations. The formalism also leads to interesting connections to the single impurity problem in Luttinger liquids. A rich structure is found in the spectral function whenever spin isotropy is broken, suggesting the presence of exotic momentum dependence in photoemission spectra of (quasi) one dimensional materials.
\end{abstract}

\section{INTRODUCTION}

The spectral properties of a single hole in a quantum antiferromagnet still represent an outstanding problem in the physics of strongly correlated electron systems. Although more than 25 years elapsed since the seminal work of Brinkman and Rice [1] (BR) there is still no consensus on the nature of hole motion (coherent or incoherent) or on the features of the long range distortion induced by the hole on the antiferromagnetic ordering. On the other hand, a full understanding of the dynamics of a single hole is clearly required before the problem of the hole-hole effective interaction, mediated by the magnetic background, can be addressed. This issue, relevant in the low doping regime, is a key problem in the framework of high temperature superconductivity. Furthermore, recent developments in angle resolved photoemission and inverse photoemission experiments [2, [4, have made possible to extract the momentum dependent spectral function in several compounds, including high temperature superconducting materials at stoichiometric composition, which are good quantum antiferromagnets. The photoemitted electron leaves a mobile hole in the spin background: therefore these experimental studies directly address the problem of hole propagation in systems where electron correlations play a key role.

A widely accepted model to describe the physics of a quantum antiferromagnet is the well known Hubbard model [5] at half filling (one electron per site):

$$
\hat{H}=-t \sum_{<i, j>}\left(c_{i \sigma}^{\dagger} c_{j \sigma}+\text { h.c. }\right)+U \sum_{i}\left(n_{i \uparrow}-\frac{1}{2}\right)\left(n_{i \downarrow}-\frac{1}{2}\right)
$$

where $c_{i \sigma}^{\dagger}\left(c_{i \sigma}\right)$ is the creation (annihilation) operator of an electron with spin $\sigma$ at the lattice site $i$ and the symbol $<i, j>$ indicates nearest neighbor summations over an hypercubic bipartite lattice in arbitrary spatial dimension $d$. Henceforth even (odd) values for $i$ indicate conventionally one of the two sublattices. The operator $n_{i \sigma}=c_{i \sigma}^{\dagger} c_{i \sigma}$ is the number operator of a particle with spin $\sigma$ at the given site $i$. This model is defined in a finite lattice with $L$ sites and standard periodic boundary conditions. When the total number of particles $N_{c}=N_{\uparrow}+N_{\downarrow}$ equals the number of sites, (i.e. at $\rho=N_{c} / L=1$ ) this Hamiltonian is believed to develop a gap in the charge excitation spectrum. This is actually rigorous in one dimension where the exact Lieb and Wu solution [6] yields a finite gap for arbitrary repulsion $U>0$ and magnetization per site $\mu=\frac{N_{\uparrow}-N_{\downarrow}}{2 L}$. A particle hole transformation

$$
c_{i \uparrow}^{\dagger} \rightarrow(-1)^{i} c_{i \uparrow}
$$

maps the half filled Hubbard model at $U>0$ and magnetization $\mu$ into the same model at $\mu^{\prime}=0$, negative interaction $\left(U^{\prime}=-U\right)$ and density $\rho^{\prime}=1-2 \mu$. This mapping also shows that the problem of a single hole in an antiferromagnet 
at non zero magnetic field is relevant to understand the photoemission spectra in superconductors: in fact, there is quite a robust numerical evidence [7] that the negative $U$ model is an $s$-wave superconductor in $d=2$, and in one dimension the exact Bethe ansatz solution predicts quasi-long range order in the ground state [8].

Although the treatment of a single hole $\left(N_{c}=L-1\right)$ might seem a major simplification, there are only few results valid and accepted in more than one dimension. The Nagaoka theorem is a remarkable exception, stating that for $d>1$ the ferromagnetic state with maximum total spin $S=\frac{1}{2}(L-1)$ is the unique ground state of the infinite $U$ Hubbard model, apart for the trivial degeneracy of the $2 S+1$ spin components [9].

At strong coupling the Hubbard model at half filling is mapped into a standard Heisenberg model with antiferromagnetic superexchange $J=\frac{4 t^{2}}{U}$. The presence of a single hole modifies slightly this mapping: each site is singly occupied and the hole hops from site to site weakening local antiferromagnetic correlations. This process is described by the so called $t-J$ model:

$$
\hat{H}=-t \sum_{<i, j>, \sigma}\left(c_{i \sigma}^{\dagger} c_{j \sigma}+h . c .\right)+J \sum_{<i, j>}\left(\vec{S}_{i} \cdot \vec{S}_{j}-\frac{1}{4} n_{i} n_{j}\right) .
$$

where the constraint of no double occupancy is understood. The exact mapping from Hubbard to $t-J$ also includes a three site term, which is neglected here because is believed not to change the physics of the model, at least in one dimension [10,11]. The last, density dependent, contribution in Eq. (1.3) can be also dropped for our purposes because it is effective only when more than one hole is present in the system.

This work extends and develops the analysis presented in a previous Letter [12] about the spectral properties of hole motion in one dimensional models of correlated electrons. In such a case, spin charge decoupling allows to describe the low energy physics by an effective Hamiltonian $\hat{H}$ written as the sum of two commuting parts $\hat{H}=\hat{H}_{\sigma}+\hat{H}_{\rho}$, the former governing the spin degrees of freedom (spinons) and the latter the charge ones (holons).

This picture is by no means new in the field of one dimensional electron systems: standard analytical treatments show that, if the excitation spectrum of the model is gapless, the low energy physics in both charge and spin sectors is described by a Luttinger liquid model [13 1.5] for generic microscopic Hamiltonians. The case is different in the Hubbard model for positive $U$ at half filling or, for negative $U$, at zero magnetic field and arbitrary density: Only one of the two sectors is gapless and the renormalization group (RG) equations of the generic (the so called $g-$ ology) model flow to strong coupling. The $g$-ology model parameterizes the most general low energy interaction present in one dimensional, translationally invariant systems of spin one-half electrons. The model depends on several coupling constants $g_{i}$, with $i=1, \ldots 4$, which may also have a spin dependence $\|$ and $\perp$ for electrons interacting with the same or with opposite spins, respectively [13. Conventionally, $g_{1}$ refers to backward scattering, $g_{2}$ and $g_{4}$ to forward scattering and $g_{3}$ to Umklapp scattering, the latter present only in lattice models at commensurate fillings. When the coupling constants $g$ 's go to strong coupling under the RG flow, they should cross the exactly solvable Luther-Emery line leading to a spin gap for $g_{1 \|}<\left|g_{1 \perp}\right|$ and a charge gap for $-2 g_{2}<\left|g_{3}\right|$. The Hubbard model at half filling belongs to the first class at negative $U$ and to the second at positive $U$, thereby providing a model Hamiltonian which encompasses the most general strong coupling fixed points. Therefore, understanding the Hubbard model at half filling and arbitrary magnetization would shed light on the physical behavior at the Luther-Emery fixed point. This would be particularly valuable for the dynamical properties because the retarded Green function

$$
G_{\sigma}(p, \omega)=-<\Psi\left|c_{p \sigma}^{\dagger}\left(\omega-\hat{H}+E_{0}+i \eta\right)^{-1} c_{p \sigma}\right| \Psi>
$$

of the Luther-Emery model is not exactly known, although a widespread prejudice ascribes no interesting features to this correlation function [8,16] due to the presence of a gap in the excitation spectrum.

A first study of the single hole Green function in quantum antiferromagnets (QAF) was performed by Brinkman and Rice [1] who calculated the Green function of the $t-J$ model by neglecting quantum fluctuations, i.e. by replacing the spin exchange interaction by its Ising form: $\vec{S}_{i} \cdot \vec{S}_{j} \rightarrow S_{i}^{z} S_{j}^{z}$ in Eq.(1.3). In the absence of holes, the Néel state is the classical ground state of the model, whereas the lowest spin excitation has a gap $\sim J$. This Hamiltonian can be thought of as the strong coupling limit of a model characterized by a gap both in the spin and in the charge sectors (say the half filled Hubbard model with uniaxial spin anisotropy). The problem of hole motion in this system has been studied in any dimensions, but its solution in $d=1$ is particularly simple and instructive. When the hole hops in the lattice it leaves a defect in the Néel background. As a result, the spectral weight $A(\omega, p)=\frac{1}{\pi} \operatorname{Im} G(p, \omega) \operatorname{shows}$ a delta function contribution at the lowest excitation energy with non zero quasiparticle weight $Z$, together with an incoherent band separated by a gap. A brief discussion of these results is contained in Section $\amalg$. The situation is similar in the limit of infinite dimensions [17] where there is no incoherent contribution and only a series of $\delta$ function peaks at higher energies is left. The inclusion of quantum fluctuations, however, drastically changes this simple picture due to the presence of gapless excitations in the magnetic background. This is the subject of the present study which we organized as follows. 
In Section III an extremely useful mapping between the one hole Hamiltonian and an effective spin problem is given in some detail. In Section [V] we discuss an interesting relationship, which emerges from the previous formulation, between hole dynamics and the impurity problem in Luttinger liquids. The main result of the present work can be summarized in the general structure we find in the Green function of all the models we have examined:

$$
G(p, \tau)=\int \frac{d Q}{2 \pi} G_{h}(p+Q, \tau) Z_{p}(Q, \tau)
$$

where $G_{h}(k, t)$ is just a free propagator for the holon: $\operatorname{Im} G_{h}(k, \omega)=\pi \delta\left(\omega-\epsilon_{h}(k)\right), \epsilon_{h}$ being the holon dispersion energy. The function $Z(Q, \tau)$ is completely determined by the spinon gapless excitations, and is highly non trivial with momentum dependent power law singularities and branch cuts. This decomposition is introduced in Section $\mathrm{V}$ which also contains a discussion of the analytical properties of the function $Z(Q, \tau)$. Few specific examples, i.e. $t-J_{X Y}$ model and the Bethe ansatz soluble models, are presented in Sections VI and VII respectively. A numerical evaluation of the non universal features of the spectral function in the Hubbard model is carried out in Section VIII while some conclusions are drawn in Section IX.

\section{HOLE MOTION IN ISING ANTIFERROMAGNETS}

The problem of hole motion in an Ising antiferromagnet can be solved exactly and provides the simplest model of hole dynamics in a magnetic environment characterized by a discrete excitation spectrum. This feature turns out to be responsible for the different behavior between the Ising and the XY case which will be discussed in Section $\mathrm{VI}$. The Hamiltonian describing hole hopping in an Ising model is a simple generalization of the $t-J$ model where only the $S^{z}$ component of the spins is retained [5]:

$$
\hat{H}=-t \sum_{i \sigma}\left[c_{i \sigma}^{\dagger} c_{i+1 \sigma}+c_{i+1 \sigma}^{\dagger} c_{i \sigma}\right]+J \sum_{i} S_{i}^{z} S_{i+1}^{z}
$$

with $J>0$. We consider a chain with an even number $L$ of sites and periodic boundary conditions. At half filling the ground state is a classical Néel state $|\mathcal{N}\rangle$. Out of the two states obtained by translation of one lattice vector we select the one with a spin down at the origin. The annihilation of the spin down electron at the origin defines our starting state $\left|0>=c_{0 \downarrow}\right| \mathcal{N}>$. The Hamiltonian (2.1) acting on $\mid 0>$ generates states which, in the thermodynamic limit, can be uniquely labeled by the position of the hole $\mid R>$. In fact, for $L \rightarrow \infty$, closed paths of the hole along the ring can be neglected and the retraceable path approximation becomes exact [1]. Then, it is easy to check that

$$
\hat{H}\left|R>=-t[|R-1>+| R+1>]+\left(E_{\mathcal{N}}+\frac{J}{2}\right)\right| R>-\frac{J}{2} \delta_{R, 0} \mid R>
$$

where $E_{\mathcal{N}}$ is the energy of the Néel state. The corresponding eigenvalue equation can be easily solved in this subspace: In the thermodynamic limit, the energy spectrum consists of a bound state with energy $E_{b}=E_{\mathcal{N}}+\frac{1}{2} J-\frac{1}{2} \sqrt{J^{2}+16 t^{2}}$ and exponentially localized wavefunction $\psi_{b}(r)$. Above the bound state lies a continuum with energies labeled by the wavevector $q \in(0, \pi): E_{q}=E_{\mathcal{N}}+\frac{1}{2} J-2 t \cos q$. For every $q$ there are two degenerate wavefunctions $\psi_{q}^{ \pm}$classified according to their parity. Odd states are unaffected by the perturbation $\delta_{R, 0}$ in Eq. (2.2) because the wavefunction vanishes on site while even states, which include the bound state, have an on site probability given by:

$$
\begin{aligned}
\left|\psi_{b}(0)\right|^{2} & =\frac{J}{\sqrt{J^{2}+16 t^{2}}} \\
\left|\psi_{q}^{+}(0)\right|^{2} & =\frac{32 t^{2} \sin ^{2} q}{L\left[J^{2}+16 t^{2} \sin ^{2} q\right]}
\end{aligned}
$$

The spectral function easily follows from the aforementioned properties of the eigenfunctions. In fact, the Lehmann decomposition of $A(p, \omega)$ gives:

$$
A(p, \omega)=\sum_{s}|<s,-p| c_{p \downarrow}|\mathcal{N}>|^{2} \delta\left(\omega-E_{s}\right)
$$

where the sum is over all the one hole states $\mid s,-p>$ of momentum $-p$ (modulo $\pi$ due to the doubling of the cell induced by the antiferromagnetic ordering in $\mid \mathcal{N}>$ ). These states can be quite generally written as Bloch superpositions of eigenstates $\mid s>$ of the Hamiltonian (2.1): 


$$
\left|s,-p>=\frac{1}{\sqrt{L}} \sum_{R} e^{i p R} T^{R}\right| s>
$$

where $T^{R}$ is the $R$ sites translation operator. This identity readily gives the matrix element appearing in Eq. (2.4) in terms of the on site value of the eigenfunction $\psi_{s}(r)$ corresponding to $|s\rangle:\left\langle s,-p\left|c_{p \downarrow}\right| \mathcal{N}\right\rangle=\frac{1}{2} \psi_{s}(0)$. Therefore both the energy levels and the matrix elements are independent of $p$. This is due to the absence of fluctuations in the classical Néel state which leads to a local Green function, as already noticed by Brinkman and Rice [1] in the $J \rightarrow 0$ limit of this problem. By substituting this result into Eq. (2.4) and taking into account the form of the energy spectrum we find that, in the thermodynamic limit, the bound state is singled out because of its finite value of the on site probability and gives rise to a delta function peak. Instead the other states merge into an incoherent band:

$$
A(p, \omega)=\frac{J}{2 \sqrt{J^{2}+16 t^{2}}} \delta\left(\omega+\frac{1}{2} \sqrt{J^{2}+16 t^{2}}\right)+\frac{2}{\pi} \frac{\sqrt{4 t^{2}-\omega^{2}}}{J^{2}+16 t^{2}-4 \omega^{2}} \Theta\left(4 t^{2}-\omega^{2}\right)
$$

where $\omega$ is now measured from the reference value $E_{\mathcal{N}}+\frac{1}{2} J$ and $\Theta(x)$ is the step function. The incoherent portion has several interesting properties: it is a regular, even function of $\omega$ which vanishes at band edges $\omega= \pm 2 t$ for every nonzero value of $J$. At large $J(J>4 t)$ it shows a broad maximum at $\omega=0$ while for $J<4 t$ it has a minimum at $\omega=0$ and two symmetrical maxima appear at $\omega= \pm \frac{1}{2} \sqrt{16 t^{2}-J^{2}}$. In the $J \rightarrow 0$ limit the incoherent part develops square root singularities at band edges in agreement with the BR analysis. The shape of the spectral function for two representative values of the coupling $J$ is shown in Fig. 1.

In conclusion: The exact Green function of this problem is purely local and then the hole does not propagate in the Ising antiferromagnet. This is conventionally understood on the basis of the "string" defect that the hole creates in the antiferromagnetic ordering when it hops [1]. However, the quasiparticle weight $Z$ is finite at all non zero values of $J$ : The hole behaves as a free particle of infinite mass. This is due to the nature of the excitation spectrum of the Ising model which does not allow for gapless modes. In fact we will show that both features of $A(p, \omega)$ will be strongly modified in more realistic models of hole dynamics.

\section{THE EFFECTIVE HOLE HAMILTONIAN}

In the following we consider the $t-J$ Hamiltonian (1.3) defined on $L$ sites with periodic boundary conditions. Our task is to derive an effective spin Hamiltonian describing how the hole hopping processes perturb the antiferromagnetic background in the particular case of single hole doping. In classical physics this would correspond to a Galileo transformation from the laboratory frame to the reference frame locally at rest with respect to the hole. This transformation can be easily generalized to quantum mechanics 18 and the derivation can be performed in arbitrary dimension. As a first step we notice that the $t-J$ Hamiltonian (1.3) is translationally invariant and then any one hole state with definite momentum $k$ and spin $\uparrow$ can be written as:

$$
\left|\psi_{k}>=\frac{1}{\sqrt{L}} \sum_{R=0}^{L-1} e^{-i k R} T_{L}^{R} c_{0 \downarrow}\right| \sigma_{0}>
$$

where $\mid \sigma_{0}>$ is a suitable spin state with the spin at the origin $R=0$ fixed to $\downarrow$ and $T_{L}$ is the spin translation operator defined by the transformation property:

$$
T_{L} \vec{S}_{R} T_{L}^{-1}=\vec{S}_{R+1} .
$$

where periodic boundary conditions (PBC) over the $L$ sites are assumed in order to define the effect of translation at the rightmost site. The latter relation determines the unitary operator $T_{L}$ only up to an arbitrary phase factor. In order to fix the phase of $T_{L}$ it is enough to specify the action of the operator on a reference ("vacuum" ) state $\mid F>$. Here we follow the convention to impose that the ferromagnetic state $\mid F>$ is translationally invariant: $T_{L}|F\rangle=|F\rangle$. By substituting the representation (3.1) of the one hole state into the eigenvalue equation for the $t-J$ Hamiltonian we find that $\left|\psi_{k}\right\rangle$ is an exact eigenstate of the $t-J$ Hamiltonian if and only if $\left|\sigma_{0}\right\rangle$ is an eigenstate of the following effective spin model:

$$
H_{k}=t\left[e^{-i k} T_{\ell}+\text { h.c. }\right]+J\left[\sum_{R=1}^{\ell-1} \vec{S}_{R} \cdot \vec{S}_{R+1}\right]
$$


where the hole-translation operator $T_{\ell}$ is defined exactly as $T_{L}(3.2)$ but with $\mathrm{PBC}$ on a squeezed chain of $\ell=L-1$ sites, without the origin $R=0$ where the hole sits. $H_{k}$ is indeed defined on $\ell$ sites since the hole at the origin is decoupled from the other sites, namely $H_{k}$ commutes with the spin operator $\vec{S}_{0}$ at the origin. In the following we take the convention to set the spin at the origin with down orientation: $S_{0}^{z}=-\frac{1}{2}$. Notice that the magnetic part, proportional to $J$, represents a Heisenberg model with open boundary condition, since all the magnetic bonds connecting the spins with the hole are obviously suppressed. Instead the hole kinetic term $\hat{K}$ (i.e. the first term in $\left.H_{k}\right)$ is written in terms of the translation operator $T_{\ell}$ which enforces periodic boundary condition on the squeezed chain. In this way we effectively traced out, with no approximations, the charge degree of freedom reducing the one hole problem to a purely, non translationally invariant, spin model. The effective Hamiltonian $H_{k}$ explicitly depends on the momentum $k$ of the state showing that the distortion of the antiferromagnetic ordering does depend in a non trivial (and non local) way on hole motion.

Also the hole dynamics can be conveniently expressed in terms of the eigenstates of $H_{k}$. The spectral function of a $\downarrow$ hole is written, in Lehmann representation, as

$$
A(p, \omega)=\frac{1}{\pi} \operatorname{Im} G(p, \omega)=\sum_{s}|<s| c_{p \downarrow}|\Psi>|^{2} \delta\left(\omega-E_{s}+E_{0}\right)
$$

where $\mid \Psi>$ is the ground state of the model with no holes and $\mid s>$ represents a complete set of one hole intermediate states. The corresponding energies are respectively $E_{0}$ and $E_{s}$ while the momentum space annihilation operator is defined by

$$
c_{p \downarrow}=\frac{1}{\sqrt{L}} \sum_{R} e^{i p R} c_{R \downarrow}
$$

Note that with the adopted definitions, the Heisenberg ground state $|\Psi\rangle$ has total momentum $N \pi$, where $N$ is the number of spins up in the squeezed chain $N=S_{z}+\ell / 2$, and $S_{z}$ is the $z$-component of the total spin. As a consequence, the intermediate states $\mid s>$ must have momentum $N \pi-p$. By using the general representation (3.1) of one hole states with momentum $k$, Eq. (3.4) becomes

$$
A(p, \omega)=\sum_{s}\left|<\sigma_{s}\right| n_{0 \downarrow}|\Psi>|^{2} \delta\left(\omega-E_{s}+E_{0}\right)
$$

where now the sum runs over all the eigenstates $\mid \sigma_{s}>$ of $H_{k}$ with $k=N \pi-p$. This expression shows that the quasiparticle weight of the hole is simply expressed as the modulus square of the overlap between the Heisenberg ground state and eigenstates of the effective hole Hamiltonian.

Equation (3.3), specialized to the case of a hole of momentum $p$, can be equivalently written in a form which makes explicit connection with the problem of a Heisenberg model with a local perturbation. In fact, by adding and subtracting the additional bond operator $\hat{J}=J \vec{S}_{1} \cdot \vec{S}_{\ell}$, the magnetic part of the Hamiltonian $\hat{H}_{\sigma}$ can be made translationally invariant (on the squeezed chain):

$$
\begin{aligned}
\hat{H}_{p} & =\hat{K}+\hat{H}_{\sigma}-\hat{J} \\
& =t(-1)^{N}\left[e^{i p} T_{\ell}+\text { h.c. }\right]+J\left[\sum_{i=1}^{\ell} \vec{S}_{i} \cdot \vec{S}_{i+1}\right]-J S_{1} \cdot \vec{S}_{\ell}
\end{aligned}
$$

The only extensive, i.e. $O(L)$, term in the total hole Hamiltonian $\hat{H}_{p}$ is $\hat{H}_{\sigma}$ which coincides with the usual Heisenberg model, while the other two contributions induce $O(1)$ corrections to the total energy. Therefore, the physics of the bulk of the spin system is not affected by the presence of the hole which, at most, acts as a boundary term on the Heisenberg antiferromagnet. This can be simply understood in two limiting cases: for a static hole, since $t=0$ the Hamiltonian $\hat{H}_{p}$ becomes a Heisenberg model with open boundary conditions, while, for $J \rightarrow 0$, the last term $\hat{J}$ is irrelevant and the eigenstates of $\hat{H}_{p}$ are those of a Heisenberg model with periodic boundary conditions on a $\ell$ site chain. A close connection between the presence of the hole and a change in boundary conditions on the magnetic Hamiltonian thus emerges quite naturally, in every dimensions, within this formalism.

In one dimension we can proceed further by mapping this spin $\frac{1}{2}$ model into a spinless fermion Hamiltonian via standard Jordan-Wigner transformation [8]. It is convenient to introduce an additional phase factor in the fermion creation operators $\psi_{n}^{\dagger}$ in order to obtain the usual sign of the kinetic energy term:

$$
S_{n}^{+}=(-1)^{n} \psi_{n}^{\dagger} \exp \left[i \pi \sum_{j=1}^{n} \psi_{j}^{\dagger} \psi_{j}\right]
$$


The magnetic part $\hat{H}_{\sigma}$ of the Hamiltonian (3.7) becomes:

$$
\hat{H}_{\sigma}=-\frac{J}{2} \sum_{i=1}^{\ell}\left(\psi_{i}^{\dagger} \psi_{i+1}+\text { h.c. }\right)+J \sum_{i=1}^{\ell}\left(\frac{1}{2}-\psi_{i}^{\dagger} \psi_{i}\right)\left(\frac{1}{2}-\psi_{i+1}^{\dagger} \psi_{i+1}\right)
$$

where the boundary conditions in the first term are periodic or antiperiodic for even or odd values of $L+N$, i.e. $\psi_{\ell+1} \equiv(-1)^{N+L} \psi_{1}$. As usual, $N$ is the number of fermions, which is related to the total magnetization of the original spin model by $S_{z}=N-\frac{\ell}{2}$. Analogously, the bond term $\hat{J}$ is written as:

$$
\hat{J}=-\frac{J}{2}(-1)^{N+L}\left(\psi_{1}^{\dagger} \psi_{\ell}+\text { h.c. }\right)+J\left(\frac{1}{2}-\psi_{1}^{\dagger} \psi_{1}\right)\left(\frac{1}{2}-\psi_{\ell}^{\dagger} \psi_{\ell}\right)
$$

In order to express the hole kinetic term $\hat{K}$ in terms of the spinless fermion operators we have to relate the spin translation operator $T_{\ell}$ to the usual fermion translation operator $T_{f}$ which leaves invariant the fermionic vacuum state and satisfies $T_{f} \psi_{i} T_{f}^{\dagger}=\psi_{i+1}$. Keeping track of the phase factors in the definitions we have $T_{\ell}=(-1)^{N} T_{f}$. The fermion translation operator is then conveniently expressed in terms of the Fourier transformed operators $\psi_{k}=$ $\ell^{-1 / 2} \sum_{j=1, \ell} \psi_{j} e^{i k j}$ where the momenta $k$ are quantized according to the choice of boundary condition:

$$
T_{\ell}=(-1)^{N} T_{f}=\exp \left[i \sum_{k}(k+\pi) \psi_{k}^{\dagger} \psi_{k}\right]
$$

By use of Eqs. 3.9.3.11) we can express the Hamiltonian of a spin down hole of momentum $p$ in the $t-J$ model, $\hat{H}_{p}$ (3.7), in terms of spinless fermion operators:

$$
\hat{H}_{p}=t \exp \left[i p+i \sum_{k} k \psi_{k}^{\dagger} \psi_{k}\right]+\text { h.c. }-\frac{J}{2} \sum_{i=1}^{\ell-1}\left(\psi_{i}^{\dagger} \psi_{i+1}+\text { h.c. }\right)+J \sum_{i=1}^{\ell-1}\left(\frac{1}{2}-\psi_{i}^{\dagger} \psi_{i}\right)\left(\frac{1}{2}-\psi_{i+1}^{\dagger} \psi_{i+1}\right)
$$

This concludes our formal manipulations on the original problem. We now have an interacting fermion system which can be studied by means of the powerful techniques developed in the framework of one dimensional physics, ranging from renormalization group approaches to bosonization methods [13]. However, before addressing these issues, it is instructive to dwell on the similarities between the problem of hole motion and the effects of local perturbations in Luttinger liquids, which emerge naturally from the structure of the effective Hamiltonian (3.7).

\section{RELATIONSHIP TO THE IMPURITY PROBLEM}

In this Section we like to investigate the close relationship between the physics of a single hole in the $t-J$ model and the impurity problem in Luttinger liquids. The connection between these two different problems becomes apparent when we take advantage of the previously discussed Galileo transformation. The Hamiltonian $\hat{H}_{p}$ governing the dynamics of the hole represents a Heisenberg model with open boundary conditions plus the hole kinetic contribution which involves the translation operator $T_{\ell}$. Furthermore, note that the matrix elements appearing in the spectral function (3.6) are related, through Eq. (3.1), to the overlaps between the ground state $|G\rangle$ of the unperturbed Hamiltonian $(\hat{J}=\hat{K}=0)$ on $L$ sites and that of the perturbed one $(\hat{J} \neq 0)$ in the squeezed chain of $\ell$ sites. The difference in the number of sites of the chain defining the perturbed state can also be thought of as the local perturbation induced by the removal of the two bonds connecting the origin in the $L$ site ring.

Let us focus our attention on the Hamiltonian of a hole of momentum $p$ in the $L$-site $t-J$ model with $X Y$ spin anisotropy: the $t-J_{X Y}$ chain. In fermion representation $\hat{H}_{p}$ is:

$$
\hat{H}_{p}=t \exp \left[i p+i \sum_{k} k \psi_{k}^{\dagger} \psi_{k}\right]+\text { h.c. }-\frac{J}{2} \sum_{i=1}^{\ell}\left[\psi_{i}^{\dagger} \psi_{i+1}+\text { h.c. }\right]+\frac{J^{\prime}}{2}(-1)^{N+L}\left[\psi_{\ell}^{\dagger} \psi_{1}+\text { h.c. }\right]
$$

where $J^{\prime}=J$ represents the perturbation induced by the hole. In order to be specific, we consider the model with $N=2 \nu+1$ fermions (spinons) in a lattice of $L-1=\ell$ sites. The kinetic operator $\hat{K}$ commutes with the magnetic term in $\hat{H}_{\sigma}$ and therefore, at $J^{\prime}=0$, the eigenvectors of $\hat{H}_{p}$ coincide with those of the $X Y$ model on $\ell$ sites with PBC while the eigenvalues are shifted by $2 t \cos (p+Q)$ where $Q$ is the momentum of the spin state. The physics of hole 
motion is contained in the perturbation term proportional to $J^{\prime}$, therefore it is convenient to study the effects of the local perturbation as a function of its strength $J^{\prime}$. In the static limit $(t=0)$ the hole kinetic term $\hat{K}$ is suppressed and we recover the Hamiltonian of a $X Y$ model with a weak bond. This problem has been extensively studied in the past [19,20]: the last term in Eq. (4.1) is a relevant perturbation and at long wavelengths the system behaves as a chain with a missing bond. As a consequence, the overlap $\zeta$ between the state with $J^{\prime}=0$ and the state with finite $J^{\prime}$ goes to zero in the thermodynamic limit with a universal exponent $X$ independent of the strength of the perturbation:

$$
\zeta=<J^{\prime} \mid G>\propto L^{-X}
$$

According to conformal field theory, the exact value of the exponent $X$ can be found analytically by taking the difference between the size corrections of the open and the periodic chain and dividing by $2 \pi v_{s}$, where $v_{s}$ is the spinon Fermi velocity. In the XY model we get $X=\mu^{2} / 4+1 / 16$ where $\mu \in(-1 / 2,1 / 2)$ is the magnetization of the XY model along the $z$ direction.

The recoil of the hole, embodied in the hole kinetic term $\hat{K}$, qualitatively changes this picture as we already anticipated. The perturbation $J^{\prime}$ is now marginal: it does not drive the model towards open boundary conditions but instead it changes the boundary conditions of the fermionic model in Eq. (4.1) introducing phase shifts for right and left moving spinons which depend on the strength of the perturbation $J^{\prime}$ and vanish as $J^{\prime} \rightarrow 0$.

The effect of the kinetic term can be understood by use of first order perturbation theory in the parameter $J^{\prime}$ in Eq. (4.1). For $J^{\prime}=0$ the ground state $\mid G>$ is non degenerate and can be represented by a Slater determinant of plane waves with PBC. At first order, the ground state becomes:

$$
\left|J^{\prime}>=\right| G>+\frac{J^{\prime}}{2 \ell} \sum_{k \neq q}\left(e^{i k}+e^{-i q}\right) \frac{<k, q\left|\psi_{k}^{\dagger} \psi_{q}\right| G>}{\epsilon_{k, q}} \mid k, q>
$$

where the sum runs over the momenta of particle-hole excitations of the unperturbed system and $\epsilon_{k, q}$ are the corresponding excitation energies:

$$
\epsilon_{k, q}=J[\cos q-\cos k]+2 t[\cos (p+k-q)-\cos p]
$$

The matrix element in Eq. (4.3) $<k, q\left|\psi_{k}^{\dagger} \psi_{q}\right| G>$ is unity provided $q$ belongs to the Fermi sea while $k$ lies outside the Fermi surface. Perturbation theory fails in the thermodynamic limit due to low energy excitations which may be of "forward" (i.e. $q \sim \pm k_{F}$ and $k \sim \pm k_{F}$ ) or "backward" type (i.e. $q \sim \pm k_{F}$ and $k \sim \mp k_{F}$ ). However, for any $t \neq 0$ the backward scattering is cut off by the recoil and does not introduce singularities in perturbation theory, as can be easily checked by use of Eq. (4.4). Instead, forward scattering is always singular leading to the vanishing of the overlap $\zeta(4.2)$ in the thermodynamic limit.

In order to better understand the change in the state induced by the boundary term $J^{\prime}$, let us consider a different problem, namely, the way the ground state changes due to a weak modification of the boundary conditions. Let us take as unperturbed state a Slater determinant of plane waves $\phi_{q}(r)=e^{i q r} \ell^{-1 / 2}$ with momentum quantization appropriate to PBC: $\ell q_{j}=2 \pi j$. A change in boundary conditions involves the introduction of certain momentum dependent phase shifts $\delta_{k}$. Therefore, we take as perturbed state another Slater determinant of plane waves $\psi_{k}(r)$ with generalized momentum quantization rule: $\ell k_{j}=2 \pi\left(j+\delta_{k}\right)$. Here and in the following, the phase shifts will be measured in units of $2 \pi$. For weak perturbations, the phase shifts will be small and we can keep only $O\left(\delta_{k}\right)$ terms in the expansion of the state in the unperturbed basis. As usual, to linear order, only a single particle-hole excitation $\mid k, q>$ is allowed and the weight of the corresponding contribution turns out to be:

$$
\frac{\pi \delta_{k}}{\ell \sin \frac{k-q}{2}}
$$

for $k \neq q$. Again, we see that a change in boundary conditions leads to singularities only for forward scattering as in the hole problem when the effect of the recoil is taken into account. The comparison can be made quantitative by linearizing the momentum dependence about the Fermi points in Eqs. (4.5,4.3) and matching the two expressions. The effective phase shifts induced by the perturbation $J^{\prime}$ at long wavelength in the Hamiltonian 4.1) are then given to $O\left(J^{\prime}\right)$ by

$$
2 \pi \delta_{ \pm}=\frac{J^{\prime} \cos k_{F}}{2 t \sin p \mp J \sin k_{F}}
$$

at the Fermi points. The two signs refer to the right and left moving spinons which in general have different phase shifts. Notice that the resulting phase shifts depend, in general, on the density as well as on the total momentum 
$p$ of the hole. Following the well known analysis of Anderson's orthogonality catastrophe [21], we then find that the overlap between the states before and after the perturbation should vanish in the thermodynamic limit with an exponent $X=\left(\delta_{+}^{2}+\delta_{-}^{2}\right) / 2$. Few details are reported in appendix (A).

In conclusion, we can interpret the effects of the presence of a hole in an antiferromagnetic background as a change of boundary conditions in the corresponding spin problem. If the recoil of the hole is neglected, (i.e. $t=0)$ both forward and backward scattering between the holon and the spinons are relevant. In this case, the hole effectively breaks the spin ring and at low energy the model becomes equivalent to an open spin chain (in the hole reference frame). Instead, for $t \neq 0$ the backward scattering channel is cut off and the perturbation induced by the hole becomes marginal. The presence of the holon introduces, via the forward scattering channel, phase shifts in the boundary conditions of the spin chain. Spinons can then propagate through the site where the hole sits.

These results have been obtained analytically for a $X Y$ model but, in view of the universality of the Luttinger Liquid description of $1 \mathrm{D}$ correlated systems, we expect that our picture remains valid for general $X X Z$ spin chains, including the isotropic Heisenberg point.

\section{FIELD THEORETICAL ANALYSIS}

In this Section we address the problem defined by the Hamiltonian $\hat{H}_{p}(3.12)$ in fermionic representation by use of the bosonization method. The bulk term $\hat{H}_{\sigma}(3.9)$ just represents a Heisenberg model with periodic boundary conditions which has been extensively studied in the past [13]. The renormalization group approach applied to such a problem shows that both backward scattering and Umklapp scattering terms are marginally irrelevant: at long wavelength the model is characterized only by the interactions of the forward type with non universal coupling constants renormalized by the RG flow. This fixed point Hamiltonian represents a Luttinger model which can be exactly mapped into a free bosonic system following the procedure of Mattis and Lieb [22], the bosons representing density fluctuations. As suggested by the analysis of Section IV, the additional terms present in $\hat{H}_{p}$ slightly modify this picture, appropriate for the undoped antiferromagnet. The combined effect of $\hat{K}$ and $\hat{J}$ on the long wavelength physics is equivalent to a change in the boundary conditions of the Luttinger model from periodic to skew: Although the original problem (3.7) is not translationally invariant due to the bond term $\hat{J}$, the low energy fixed point Hamiltonian develops effective boundary conditions which depend on the non universal parameters characterizing the microscopic model. In particular we expect that the boundary conditions will continuously evolve from periodic at small $J / t$ to generally skew at finite $J / t$ with the exception of the zero magnetic field case where the boundary conditions at the fixed point remain periodic for any $t \neq 0$ due to the presence of the additional $S U(2)$ spin symmetry, as will be shown later. The only singular point is at $t=0$ where the hole kinetic contribution vanishes and $\hat{H}_{p}$ describes a Heisenberg model with open boundary conditions. In the following, we will exploit this picture extracting quantitative predictions which will be later compared with the exact solution in specific models.

On this basis we are led to consider a low energy problem defined by a long wavelength effective Hamiltonian $\hat{H}_{p}$ sum of two commuting terms: a Luttinger model with skew boundary conditions plus the hole kinetic term

$$
\begin{aligned}
\hat{K} & =\epsilon_{h}\left(k_{h}\right) \\
k_{h} & =p+Q
\end{aligned}
$$

Here we have defined $k_{h}$ as the holon momentum and $Q$ is the spinon momentum which obeys the quantization rule appropriate for the skew boundary conditions of $\hat{H}_{\sigma}$. Eq. (5.2) therefore represents momentum conservation of the charge and spin excitations. Generally, we expect that the renormalization group flow modifies the effective hole band in a non universal way: $2 t \cos \left(k_{h}\right) \rightarrow \epsilon_{h}\left(k_{h}\right)$.

Clearly, such a form of the long wavelength Hamiltonian may describe only the low energy part of the excitation spectrum of the effective hole Hamiltonian (3.12). Therefore, an elementary spin excitation which changes the total spinon momentum $Q$ must be accompanied by a corresponding variation of the total momentum $p$ so that the holon momentum $k_{h}$ is not modified. Otherwise the hole kinetic term $\hat{K}$ would contribute a finite amount to the excitation energy and a low energy excitation in the spin channel would not correspond to a low lying excitation of the total Hamiltonian. In other words, the low energy spin excitations described by the effective spinon Hamiltonian with skew boundary conditions represent the physical excitation spectrum at fixed holon momentum $k_{h}$.

The overall picture of the low energy physics of the one hole problem is confirmed by the exact solution of Bethe Ansatz models which also allows for a quantitative analysis of their long wavelength properties as will be shown later.

As a first step, we now generalize the bosonization procedure in Luttinger models with skew boundary conditions (Sec. $\mathrm{VA}$ ), then we analyze in some detail the form of the hole kinetic operator present in the effective Hamiltonian, the possible boundary conditions which may occur in microscopic models and the corresponding finite size corrections 
to the ground state energy of this effective model (Sec. $\mathrm{VB}$ ). Finally we discuss some implication of the previously obtained results including the expected asymptotic form of the hole Green function (Sec. $\mathrm{VG}$ ).

\section{A. The long wavelength Hamiltonian: Bosonization}

At low energy, the important degrees of freedom for a many fermion system are those close to the Fermi momenta $k_{F}^{ \pm}$for the left and right movers, i.e. $k \sim+k_{F}^{+}$in the right $(+)$branch and $k \sim-k_{F}^{-}$in the left $(-)$branch. As usual, the two branches are extended to infinity, within the assumption that the low energy physics is not affected by this approximation [13]. This extension allows to define two fields $\psi_{+}(x)$ and $\psi_{-}(x)$ representing, in the continuum limit, the annihilation operators for spinless fermions on the right and left branch. The continuum limit of the original fermionic field $\psi^{\dagger}(x)$ is then given by the linear combination:

$$
\psi^{\dagger}(x) \propto e^{i k_{F}^{+} x} \psi_{+}^{\dagger}(x)+e^{-i k_{F}^{-} x} \psi_{-}^{\dagger}(x)
$$

where we have kept the distinction between right and left Fermi momenta $k_{F}^{ \pm}$which may in principle differ. The origin of the fermionic field $x=0$ has been chosen to match with the first site $i=1$ of the squeezed chain of length $\ell$. As stated before, the fields $\psi_{ \pm}(x)$ obey skew boundary conditions:

$$
\psi_{ \pm}(x+\ell)=e^{2 \pi i \delta_{ \pm}} \psi_{ \pm}(x)
$$

with arbitrary phase shifts $\delta_{ \pm}$.

The Luttinger model is defined by right and left moving fermions with kinetic term and interactions of the forward type. This allows to express the effective low energy theory in terms of two bosonic fields defined as bilinear combinations of the fermionic operators. More precisely, following Mattis and Lieb [22], the Fourier transform $N_{ \pm}(q)=\int d x e^{-i q x} N_{ \pm}(x)$ of the operators $N_{ \pm}(x)=\psi_{ \pm}^{\dagger}(x) \psi_{ \pm}(x)+$ const. satisfies non trivial commutation rules

$$
\left[N_{ \pm}(q), N_{ \pm}(-q)\right]=\mp \frac{\ell q}{2 \pi}
$$

The above equations suggest the definition of the boson field $\Phi(x)$ and its conjugate momentum $\Pi(x)$ via:

$$
\begin{aligned}
& N_{+}(x)=\psi_{+}^{\dagger}(x) \psi_{+}(x)-<\psi_{+}^{\dagger}(x) \psi_{+}(x)>=\frac{1}{\sqrt{4 \pi}}\left(\Pi(x)+\partial_{x} \Phi(x)\right) \\
& N_{-}(x)=\psi_{-}^{\dagger}(x) \psi_{-}(x)-<\psi_{-}^{\dagger}(x) \psi_{-}(x)>=-\frac{1}{\sqrt{4 \pi}}\left(\Pi(x)-\partial_{x} \Phi(x)\right)
\end{aligned}
$$

where the average $\langle>$, taken on the reference ground state of the Luttinger model, is introduced in order to regularize the divergences. These operators obey the canonical commutation relations:

$$
\left[\Phi(x), \Pi\left(x^{\prime}\right)\right]=i \delta\left(x-x^{\prime}\right)
$$

Notice that the densities $N_{ \pm}(x)$ satisfy periodic boundary conditions independently of the phase shifts $\delta_{ \pm}$, being bilinear combinations of fermionic operators. As a consequence the fields $\partial_{x} \Phi(x)$ and $\Pi(x)$ obey periodic boundary conditions and the Luttinger Hamiltonian, which can be entirely expressed in terms of the bosonic fields $(\Phi(x), \Pi(x))$, has exactly the same gaussian structure as for a pure Heisenberg model:

$$
\hat{H}_{\sigma}=\frac{v_{s}}{2} \int_{0}^{\ell} d x\left[K_{\sigma} \Pi^{2}(x)+\frac{1}{K_{\sigma}}\left(\partial_{x} \Phi\right)^{2}\right]+\mathrm{const}
$$

where $v_{s}$ and $K_{\sigma}$ are the renormalized Fermi velocity and the dimensionless interaction parameter which characterize the long wavelength behavior of the Heisenberg model [ [8].

This simple quadratic Hamiltonian can be diagonalized by introducing normal modes and this procedure leads to the familiar Luttinger Liquid energy spectrum of the one dimensional Heisenberg model. However, here we are interested in the change in the finite size corrections to the energy spectrum induced by the presence of the hole. Therefore, the normal modes must be carefully defined in the $\ell$ site chain. As a first step note that two quantum numbers labeling the eigenstates can be defined: The two operators 


$$
\begin{gathered}
\Pi^{*}=\int_{0}^{\ell} \Pi(x) d x=\sqrt{\pi} \int_{0}^{\ell}\left[N_{+}(x)-N_{-}(x)\right] d x \\
\Delta \Phi=[\Phi(\ell)-\Phi(0)]=\sqrt{\pi} \int_{0}^{\ell}\left[N_{+}(x)+N_{-}(x)\right] d x
\end{gathered}
$$

commute each other and also commute with $\hat{H}_{\sigma}$, being related to the total number of fermions on the two branches, which are conserved quantities in the Luttinger model. Therefore the diagonalization of the quadratic form (5.8) can be performed in each sector defined by the pair of quantum numbers $\left(\Delta \Phi, \Pi^{*}\right)$. In particular, the choice $\Delta \Phi=\Pi^{*}=0$ identifies the reference state introduced in Eq. (5.6). The quantization rules directly follow from the definition (5.9) of these operators. At fixed total number of fermions (i.e. at fixed magnetization in the original model) $\Delta \Phi$ is uniquely determined. A low energy excitation in this model corresponds to moving a fermion from the left to the right branch. This changes the value of $\Pi^{*}$ by an even multiple of $\sqrt{\pi}$. On the other hand, by varying the total magnetization by integers, $\Delta \Phi$ changes by integer multiples of $\sqrt{\pi}$ while the model preserves a non degenerate (degenerate) ground state for even (odd) $\ell$ due to a change in the boundary conditions associated with the Jordan-Wigner transformation. Therefore we conclude that the possible values of $\Delta \Phi$ and $\Pi^{*}$ are

$$
\begin{aligned}
\Delta \Phi & =\sqrt{\pi}(n+\gamma) \\
\Pi^{*} & =2 \sqrt{\pi}(m+\delta)
\end{aligned}
$$

Here $m$ and $n$ are integers while $\gamma$ and $\delta$ are two non universal real quantities (defined $\bmod (1)$ ) which characterize the ground state of the model. Their precise value is determined by the renormalization group flow which connects the microscopic model to the Luttinger Hamiltonian. The bosonization procedure alone does not fix these quantities uniquely, except when additional symmetries are present in the microscopic model.

Now we are ready to introduce the normal modes which diagonalize the quadratic form (5.8). It is convenient to define the periodic field

$$
\Psi(x)=\Phi(x)-\frac{x}{\ell} \Delta \Phi
$$

By substituting into (5.8) we get:

$$
\hat{H}_{\sigma}=\frac{v_{s}}{2} \int_{0}^{\ell} d x\left[K_{\sigma} \Pi^{2}(x)+\frac{1}{K_{\sigma}}\left(\partial_{x} \Psi\right)^{2}\right]+\frac{v_{s}}{2 \ell K_{\sigma}}(\Delta \Phi)^{2}
$$

Next we define canonical creation and annihilation boson operators:

$$
\begin{aligned}
& \Psi_{k}=\frac{1}{\sqrt{\ell}} \int_{0}^{\ell} d x \Psi(x) e^{-i k x}=\sqrt{\frac{K_{\sigma}}{2|k|}}\left(a_{k}^{\dagger}+a_{-k}\right) \\
& \Pi_{k}=\frac{1}{\sqrt{\ell}} \int_{0}^{\ell} d x \Pi(x) e^{i k x}=i \sqrt{\frac{|k|}{2 K_{\sigma}}}\left(a_{-k}^{\dagger}-a_{k}\right)
\end{aligned}
$$

where the values of $k \neq 0$ correspond to periodic boundary conditions and are quantized in units of $\frac{2 \pi}{\ell}$. Finally we get the low energy Hamiltonian $\hat{H}_{\sigma}$ in diagonal form:

$$
\hat{H}_{\sigma}=E_{0}+\frac{v_{s}}{2 \ell}\left(\frac{(\Delta \Phi)^{2}}{K_{\sigma}}+K_{\sigma}\left(\Pi^{*}\right)^{2}\right)+v_{s} \sum_{k \neq 0}|k| a_{k}^{\dagger} a_{k}
$$

Here $E_{0}$ is the reference energy of the non degenerate ground state of the model with $\gamma=\delta=0$ which is known, from conformal field theory [8], to depend on the lattice size $\ell$ as:

$$
E_{0}=e_{0} \ell-v_{s} \frac{\pi}{6 \ell}
$$

if $e_{0}$ is the Heisenberg ground state energy per site. Notice that whenever $\delta$ or $\gamma$ acquire non zero values only the $1 / \ell$ finite size corrections to the ground state energy (5.12) are modified. The quantum numbers $\gamma$ and $\delta$ then correspond to energy contributions induced by a change in the boundary conditions of the model. This remark will be made more precise in the following. 


\section{B. Spinon momentum and skew boundary conditions}

In the previous Section we investigated the bosonization of the bulk part of the spin Hamiltonian in some detail. At the Luttinger fixed point the Hamiltonian $\hat{H}_{\sigma}$ can be expressed in terms of bosonic operators in the standard form (5.8) independently of the presence of skew boundary conditions (5.4). However, the energy spectrum depends on two quantum numbers $\left(\Delta \Phi, \Pi^{*}\right)$ which should be somehow related to the particular choice of boundary conditions. In order to make this relationship more transparent, let us analyze the bosonization form of the momentum operator (i.e. of the translation operator) appropriate to the chosen boundary conditions.

The fermionic translation operator is defined by $T_{f} \psi(x) T_{f}^{\dagger}=\psi(x+1)$. This unitary transformation can be split conveniently into two steps: first a conventional translation of left and right movers $\psi_{ \pm}(x) \rightarrow \psi_{ \pm}(x+1)$ (5.17) and then a rescaling of the fields: $\psi_{+} \rightarrow e^{-i k_{F}^{+}} \psi_{+}$and $\psi_{-} \rightarrow e^{i k_{F}^{-}} \psi_{-}$(5.16), in order to take into account the phase factors in (5.3). The correct form of $T_{f}$ is then $T_{f}=T_{1} T_{2}$ where

$$
\begin{aligned}
& T_{1}=\exp \left\{i \int_{0}^{\ell} d x\left[k_{F}^{+} \psi_{+}^{\dagger}(x) \psi_{+}(x)-k_{F}^{-} \psi_{-}^{\dagger}(x) \psi_{-}(x)\right]\right\} \\
& T_{2}=\exp \left\{-\int_{0}^{\ell} d x\left[\psi_{+}^{\dagger}(x) \partial_{x} \psi_{+}(x)+\psi_{-}^{\dagger}(x) \partial_{x} \psi_{-}(x)\right]\right\}
\end{aligned}
$$

There is no overall constant in the definition of the translation operator since the expression (5.17) acts as the identity on the fermionic vacuum state. Note that $T_{1}$ has been already bosonized via the (5.6). The other factor $T_{2}$ is just a conventional translation of the fields $\psi_{ \pm}(x)$ and acts on the bilinear forms $N_{ \pm}(x)$ as the bosonic translation operator: $\Pi(x) \rightarrow T_{2} \Pi(x) T_{2}^{-1}=\Pi(x+1)$ and $\Phi(x) \rightarrow \Phi(x+1)$. Therefore

$$
T_{2}=\exp \left\{\frac{i}{2} \int_{0}^{\ell} d x\left[\Pi(x) \partial_{x} \Phi+\partial_{x} \Phi \Pi(x)\right]\right\}
$$

From the previous analysis is clear how to represent the translation operator $T_{f}$ within bosonization. By use of the explicit expressions of the terms in $T_{1}, T_{2}$ and Eq. (5.6) we get

$$
\begin{aligned}
T_{f} & =e^{i \hat{P}} \\
\hat{P} & =Q_{0}+\frac{k_{F}^{+}+k_{F}^{-}}{2 \sqrt{\pi}} \Pi^{*}+\frac{k_{F}^{+}-k_{F}^{-}}{2 \sqrt{\pi}} \Delta \Phi+\frac{1}{2} \int_{0}^{\ell} d x\left[\Pi(x) \partial_{x} \Phi+\partial_{x} \Phi \Pi(x)\right] \\
& =Q_{0}+\left(k_{F}^{+}+k_{F}^{-}+2 \pi \frac{(n+\gamma)}{\ell}\right)(m+\delta)+\frac{k_{F}^{+}-k_{F}^{-}}{2}(n+\gamma)+\frac{1}{2} \int_{0}^{\ell} d x\left[\Pi(x) \partial_{x} \Psi+\partial_{x} \Psi \Pi(x)\right]
\end{aligned}
$$

where in the last line we took advantage of the canonical transformation (5.11) and we expressed $\Pi^{*}$ and $\Delta \Phi$ by use of Eqs. (5.10). The additive constant $Q_{0}$ represents a finite contribution which depends on the bandwidth cut-off of the Luttinger model but not on the chain length $\ell$ and is obtained by the substitution of Eq. (5.6) into Eq. (5.16). Its value is determined by requiring that in the ground state $(n=m=0)$ the spinon momentum, in the $\ell \rightarrow \infty$ limit, tends to $k_{F}$, i.e. to the momentum of the translationally invariant case without perturbation induced by the hole $\left(\hat{J}=\hat{K}=0\right.$ in Eq. 3.7). This gives $Q_{0}=k_{F}(1-2 \delta)$ for odd $\ell$. The total momentum operator $\hat{P}$ commutes with the bulk Hamiltonian $\hat{H}_{\sigma}$ and then each spinon state is characterized by a spinon momentum $Q$ which is related to the total momentum $p$ and to the holon momentum $k_{h}$ by Eq. (5.2).

Through the bosonized form of the fermion translation operator it is also possible to relate the phase shifts $\delta_{ \pm}$ which define the boundary condition of the microscopic model to the effective parameters $(\gamma, \delta)$ entering the low energy Hamiltonian. The elementary excitation which changes the value of $\Pi^{*}$ corresponds to moving a fermion from the left to the right branch. In fermionic representation, this excitation carries momentum $k_{F}^{-}+k_{F}^{+}+\frac{2 \pi}{\ell}\left(\delta_{+}-\delta_{-}\right)$. Instead, in bosonic representation, this corresponds to $n=0$ and $m=1$. In order to match the change in total momentum due to such an excitation we have to identify

$$
\gamma=\delta_{+}-\delta_{-} \quad \bmod (1)
$$


Analogously, a low energy and low momentum excitation which changes the total spin corresponds to adding one fermion to the right branch and one fermion to the left branch. The change in momentum is then $k_{F}^{+}-k_{F}^{-}+\frac{2 \pi}{\ell}\left(\delta_{+}+\delta_{-}\right)$ while the quantum numbers are $n=2$ and $m=0$. Matching the two expressions gives:

$$
\delta=\frac{\delta_{+}+\delta_{-}}{2} \bmod (1)
$$

This concludes the bosonization of the long wavelength effective Hamiltonian, which includes the bulk contribution $\hat{H}_{\sigma}$ and the hole kinetic term $\hat{K}$ which is written in terms of $T_{f}$. Collecting the various terms together our final expression for the low energy spectrum of one hole at momentum $p$ is:

$$
\begin{aligned}
E_{p} & =E_{\sigma}+E_{h} \\
E_{\sigma} & =e_{0} \ell-v_{s} \frac{\pi}{6 \ell}+\frac{2 \pi v_{s}}{\ell}\left(\frac{(n+\gamma)^{2}}{4 K_{\sigma}}+K_{\sigma}(m+\delta)^{2}\right) \\
E_{h} & =\epsilon_{h}\left(k_{h}\right) \\
k_{h} & =p+Q \\
Q & =k_{F}(2 m+1)+\frac{2 \pi}{\ell}\left[\left(\omega_{+}+n+\gamma\right)(m+\delta)+\frac{\omega_{-}}{2}(n+\gamma)\right]
\end{aligned}
$$

where we have defined $\omega_{ \pm}=\left(k_{F}^{+} \pm k_{F}^{-}\right) \frac{\ell}{2 \pi}(\bmod 1)$. In the thermodynamic limit, the quantization rule of the spinon momentum reduces to the non interacting result which, for odd $\ell$, reads

$$
Q_{m}=k_{F}(2 m+1)
$$

but the finite size corrections of energy and momentum explicitly depend on the phase shifts. We stress that both the parameters entering the effective, long wavelength Hamiltonian $\left(v_{s}, K_{\sigma}, k_{F}^{ \pm}\right)$and the phase shifts $\left(\delta_{ \pm}\right)$cannot be trivially related to the bare lattice Hamiltonian because the RG flow renormalizes all the couplings not protected by conservation laws. Their value can however be uniquely determined in Bethe Ansatz soluble model by matching the form of the finite size corrections of the one hole energy and momentum. This program will be pursued in the following Sections. More information can be gained at zero magnetization. In this case, the effective spin model (3.3) has the additional $S U(2)$ spin rotational symmetry which limits the possible boundary conditions of the Luttinger model. In fact, in spin isotropic models, the allowed boundary conditions are either open or periodic: non trivial phase shifts are not compatible with the requirement of spin isotropy. Therefore, according to our basic assumption the hole kinetic term stabilizes the periodic boundary conditions in the effective spin Hamiltonian. This observation leads to a unique determination of the phase shifts $\delta_{ \pm}$at zero magnetization which only depend on the parity of the number of sites $\ell$. We first note that the $z$ component of the total spin $S^{z}$ is simply related to the total number of fermions and then the spin excitations are labeled by the quantum number $n$ in (5.10). By definition $n=0$ corresponds to the ground state. Moreover, in zero magnetic field, states with opposite values of $S^{z}$ are degenerate. For even $\ell$, the ground state is a singlet and then excited states labeled by $n$ and $-n$ are degenerate: This implies $\gamma=0$ through Eq. (5.23). For odd $\ell$ the ground state itself is a spin doublet which gives $\gamma=1 / 2(\bmod 1)$ by the same argument. For periodic boundary conditions also the total momentum is a good quantum number. At fixed magnetization, excited states of definite momentum are labeled by $m$ in Eq. (5.10), the ground state corresponding to the choice $m=0$. For even $\ell$ the ground state is unique and excited states with opposite momentum are degenerate giving $\delta=0$ by (5.23) while, for odd $\ell$ the ground state has finite momentum which implies a twofold degeneracy due to parity. Such a degeneracy is compatible with the form of the energy spectrum (5.23) only for $\delta=1 / 2$.

As an example of the previous analysis, we plot in Fig. 2 the overlap square $Z$ between the Heisenberg ground state on a $\ell=L-1$ site ring and the ground state of a hole of momentum $p=\pi / 2$ in the $L$ site $t-J$ model at $J=4 t$ and vanishing magnetization. The results have been obtained by Lanczos diagonalizations in chains with even $L \leq 26$. According to our analysis we expect that at long wavelengths the single hole problem in an even chain is described by an effective Heisenberg Hamiltonian with $\delta=\gamma=1 / 2$ which correspond to periodic boundary conditions on $L-1$ sites, leading to a finite overlap in the thermodynamic limit. Actually, the numerical results provide quite a strong evidence in favor of this picture showing an overlap $Z$ which increases with the size of the system.

In conclusion we see that spin isotropy determines the quantization constant $\gamma$ while the occurrence of periodic boundary conditions in the effective long wavelength Hamiltonian fixes the value of $\delta$. At finite magnetization, spin isotropy is broken and we expect the occurrence of generic, momentum dependent values for $\gamma$ and $\delta$. These phase shifts will play an important role in determining the singularities of the Green function. This subject will be discussed in the following Section.

For completeness let us briefly extend the previous discussion of the energy spectrum of the model to the case of open boundary conditions. According to our assumption this case is relevant only when the hole effective hopping 
amplitude $t$ vanishes. The general form of the bulk Hamiltonian $\hat{H}_{\sigma}$ (5.8) does not depend on the choice of boundary conditions and is therefore unaltered. However, after having performed the canonical transformation (5.11), the normal modes are now defined by use of standing waves rather than the previously introduced plane waves (5.13):

$$
\begin{aligned}
& \Psi_{n}=\sqrt{\frac{2}{\ell}} \int_{0}^{l} \sin \left(k_{n} x\right) \Psi(x)=\sqrt{\frac{K_{\sigma}}{2 k_{n}}}\left(a_{k_{n}}^{\dagger}+a_{k_{n}}\right) \\
& \Pi_{n}==\sqrt{\frac{2}{\ell}} \int_{0}^{l} \sin \left(k_{n} x\right) \Pi(x)=i \sqrt{\frac{k_{n}}{2 K_{\sigma}}}\left(a_{k_{n}}^{\dagger}-a_{k_{n}}\right)
\end{aligned}
$$

where now $k_{n}=\frac{(2 n+1) \pi}{2 \ell}$ with $n \geq 0$. The bulk Hamiltonian for open boundary conditions then reads:

$$
\hat{H}_{\sigma}=E_{0}+v_{s} \sum_{n \geq 0} k_{n} a_{k_{n}}^{\dagger} a_{k_{n}}+\frac{v_{s}}{2 \ell K_{\sigma}}(n+\gamma)^{2}
$$

The final expression for the energy spectrum of the hole problem at $t=0$, i.e. when open boundary conditions apply, is then

$$
E=E_{0}+\frac{2 \pi v_{s}}{\ell} \frac{1}{4 K_{\sigma}}(n+\gamma)^{2}
$$

where now the size scaling of the reference energy is 19]

$$
E_{0}=e_{0} \ell-v_{s} \frac{\pi}{24 \ell}
$$

Again, in the spin isotropic case the values of the phase shifts are constrained by the $S U(2)$ symmetry which gives $\gamma=0$ for even $\ell$ and $\gamma=1 / 2$ for odd $\ell$.

Now we conclude this Section by expressing the expected finite size corrections to the one hole energy which emerge from our picture. On the basis of the discussion at the beginning of this Section, it is particularly convenient to work at fixed holon momentum $k_{h}$ extracting the size corrections of the low energy excitation spectrum obtained by varying the spinon momentum and the magnetization of the model. These results will be later compared with the exact form of the energy in two Bethe ansatz soluble models. The size dependence of the hole kinetic contribution (5.24) can be obtained by direct substitution of the explicit form of the spinon momentum (5.26) giving a term proportional to the charge velocity

$$
v_{c}\left(k_{h}\right)=\frac{\partial \epsilon_{h}\left(k_{h}\right)}{\partial k_{h}}
$$

Here $k_{h}$ is related to $Q_{m}$ and to the total momentum $p$ by the conservation law (5.25). We also allow for a size dependence of the total hole momentum $p$ :

$$
p=p_{0}+\frac{2 \pi \alpha}{\ell}
$$

valid up to $o(1 / \ell)$ terms. The constant $\alpha$ depends on the adopted sequence of lattice sizes. By the conservation law $k_{h}=p+Q$ (Eq. 5.25) the holon momentum acquires the finite size corrections of $p$ (Eq. 5.33) and $Q$ (Eq. 5.26). Therefore, the holon kinetic term (5.24) will contribute to the $O(1 / \ell)$ size corrections of the ground state energy as

$$
\Delta E_{h}=\frac{2 \pi v_{c}}{\ell}\left[\alpha+(\delta+m)(\gamma+n)+\omega_{+}(\delta+m)+\frac{1}{2} \omega_{-}(\gamma+n)\right]
$$

On the other hand, the spinon term, being described by the conformal field theory which characterizes the Luttinger liquids, gives rises to the finite size corrections already obtained in Eq. (5.23).

In conclusion, at fixed holon momentum $k_{h}$, the finite size corrections of the energy $E_{p}$ of the single hole problem give rise to a tower of states which depends on two quantum numbers $(n, m)$ and have total momentum $p=k_{h}-Q_{m}$ (5.27). The size corrections are uniquely determined by three bulk properties of the system $\left(v_{c}, v_{s}, K_{\sigma}\right)$ and four additional constants which determine the boundary conditions of the effective spin Hamiltonian $\left(\omega_{ \pm}, \gamma, \delta\right)$ :

$$
\begin{aligned}
\Delta E_{p} & =\frac{2 \pi v_{c}}{\ell}\left[\alpha+(\delta+m)(\gamma+n)+\omega_{+}(\delta+m)+\frac{1}{2} \omega_{-}(\gamma+n)\right] \\
& +\frac{2 \pi v_{s}}{\ell}\left[K_{\sigma}(\delta+m)^{2}+\frac{1}{4 K_{\sigma}}(\gamma+n)^{2}\right]-\frac{\pi}{6 \ell} v_{s}
\end{aligned}
$$


Through this equation we can in principle evaluate the elusive spinon phase shifts $\delta_{ \pm}$by computing the easily accessible finite size corrections of the one hole energy. This equation has been obtained in the framework of the $t-J$ model where no double occupancy is allowed but we will show that the same structure persists also in the one dimensional Hubbard model at finite $U$. Therefore we believe that this form of the finite size scaling is a general feature of one dimensional correlated models.

\section{Orthogonality catastrophe and the hole Green function}

In this Section we like to relate the previously introduced phase shifts with the behavior of physical quantities and specific dynamical correlation functions of the one hole problem. In particular, we will address first the evaluation of the hole quasiparticle weight at holon momentum $k_{h}$ and then the calculation of the asymptotic behavior of the Green function.

The quasiparticle weight is defined as the square of the modulus of the matrix element:

$$
\zeta=<k_{h}\left|c_{p, \downarrow}\right| \Psi>
$$

where $\mid k_{h}>$ is the exact one hole ground state at holon momentum $k_{h}, \mid \Psi>$ is the ground state of the Heisenberg model and we have chosen the convention of creating a hole with $\downarrow$ spin projection. Momentum conservation implies that in the ground state $k_{h}=p \pm k_{F}$, where $k_{F}$ is the spinon Fermi momentum. By use of the Galileo transformation on the hole problem and a further Jordan-Wigner transformation on the "up" spins, the problem is reduced to the evaluation of the overlap between the two fermionic states corresponding to the Heisenberg ground state $\mid \Psi>$ on a $L$ site ring and the ground state $\mid k_{h}>$ of the effective spin Hamiltonian defined on the lattice of $\ell=L-1$ sites and the same number of up spins. In order to study the behavior of the quasiparticle weight for $L \rightarrow \infty$, we can limit our attention to the long wavelength form of the effective spin Hamiltonian. The problem is therefore to compute the overlap of two eigenstates of the same bosonic Hamiltonian (5.8), with different boundary conditions: while the reference state $\mid \Psi>$ has standard periodic boundary conditions (i.e. $\Pi^{*}=\Delta \Phi=0$ ), the one hole ground state $\mid k_{h}>$ is defined by non universal, momentum dependent, values of the phase shifts:

$$
\begin{aligned}
\Pi^{*} & =2 \sqrt{\pi} \delta=\sqrt{\pi}\left(\delta_{+}+\delta_{-}\right) \\
\Delta \Phi & =\sqrt{\pi} \gamma=\sqrt{\pi}\left(\delta_{+}-\delta_{-}\right)
\end{aligned}
$$

In the continuum limit this overlap is strictly zero because the two states are eigenstates of the bosonic Hamiltonian with different eigenvalues for $\Delta \Phi$ and $\Pi^{*}$. This orthogonality is however an artifact of the Luttinger extension to a system containing an infinite number of particles and it is clear that in a finite system the overlap between two states with different boundary conditions will be in general finite. The solution is to "regularize" the Luttinger branches, in order to be consistent with a tight binding model where the same phase shifts $\delta_{+}$and $\delta_{-}$at the Fermi energy are induced by a local potential which gives no contribution to the backward scattering. In a free Fermi gas, $K_{\sigma}=1$ and the two states can be written as Slater determinants of plane waves with suitable phase shifts. In this case, the overlap can be easily calculated giving an asymptotic power law behavior which only depends on the value of the phase shifts at the Fermi energy of the right and left branches:

$$
\begin{aligned}
\zeta & =\left\langle k_{h}\right| \Psi>\propto \exp \left[-\frac{1}{2}\left(\delta_{+}^{2}+\delta_{-}^{2}\right) \ln \ell\right] \sim L^{-X_{0}} \\
X_{0} & =\frac{1}{2}\left(\delta_{+}^{2}+\delta_{-}^{2}\right)=\left(\delta^{2}+\frac{\gamma^{2}}{4}\right)
\end{aligned}
$$

where the last equality in Eq. (5.39) follows from Eq.(5.37). The formal calculation of the overlap is contained in appendix A. This formula generalizes the exact result valid for $\delta_{+}=\delta_{-}$[11,23 and agrees with the prediction of conformal field theory relating the finite size corrections of the energy to the exponent of the boundary operators [19]. In fact, the explicit expression (5.39) coincides with the term proportional to $2 \pi v_{s} / \ell$ in the final formula Eq. (5.35) for the finite size corrections of the one hole energy.

This formalism can be easily generalized to the case $K_{\sigma} \neq 1$ : In fact, the overlap does not change upon unitary transformations and it is known that the scaling of the bosonic fields

$$
\begin{aligned}
\Pi^{\prime}(x) & =\sqrt{K_{\sigma}} \Pi(x) \\
\Phi^{\prime}(x) & =\frac{1}{\sqrt{K_{\sigma}}} \Phi(x)
\end{aligned}
$$


maps the interacting problem with $K_{\sigma} \neq 1$ to the one with $K_{\sigma}=1$. By the same transformation, the boundary condition for the one hole state are modified due to Eqs. (5.9,5.10): $\gamma^{\prime}=\gamma / \sqrt{K_{\sigma}}, \delta^{\prime}=\delta \sqrt{K_{\sigma}}$, while for the reference state the boundary condition are unchanged. The effective phase shifts of the parent non interacting case then follow immediately:

$$
\begin{aligned}
& \delta_{+}^{\mathrm{eff}}=\frac{1}{2}\left[2 \sqrt{K_{\sigma}} \delta+\frac{1}{\sqrt{K_{\sigma}}} \gamma\right] \\
& \delta_{-}^{\mathrm{eff}}=\frac{1}{2}\left[2 \sqrt{K_{\sigma}} \delta-\frac{1}{\sqrt{K_{\sigma}}} \gamma\right]
\end{aligned}
$$

leading to the general expression:

$$
X_{0}=\left[K_{\sigma} \delta^{2}+\frac{1}{4 K_{\sigma}} \gamma^{2}\right]
$$

again consistent with the finite size corrections to the energy (5.35).

The relation between the finite size corrections to the energy and the exponent $X_{0}$ of the orthogonality catastrophe is a general property of all conformal field theories [19] and holds also in other cases. For instance, the exponent associated to the open boundary fixed point can be determined in terms of the finite size corrections obtained in Eq. (5.30):

$$
X_{0}=\frac{\gamma^{2}}{4 K_{\sigma}}+\frac{1}{16}
$$

where use has been made of the known additional contributions to the ground state energy with periodic (5.15) and open (5.31) boundary conditions on a $L$ and $\ell$ site chain respectively 19. This expression, which applies in the limit of a static hole, yields the exact exponent $X_{0}=3 / 16$ in the isotropic case, when $K_{\sigma}=1 / 2$ and $\gamma=1 / 2$. This has been checked numerically in Fig. 3 by Lanczos diagonalization of the hole problem in the limit of vanishing hopping amplitude $t$.

Now we can proceed to the study of the asymptotic behavior of the retarded single hole Green function:

$$
G_{\sigma}(p, \tau)=i<\Psi\left|c_{p, \sigma}^{\dagger} e^{-i(\hat{H}-i \eta) \tau} c_{p, \sigma}\right| \Psi>
$$

valid for $\tau>0$ with $\eta=0^{+}$as convergence factor. As usual, by performing the Galileo transformation and then a Jordan-Wigner transformation we can map the problem of the evaluation of the Green function to the calculation of the purely fermionic matrix element:

$$
\begin{aligned}
& G_{\uparrow}(p, \tau)=i<\Psi\left|e^{-i\left(\hat{H}_{p}-i \eta\right) \tau} n_{0}\right| \Psi> \\
& G_{\downarrow}(p, \tau)=i<\Psi\left|e^{-i\left(\hat{H}_{p}-i \eta\right) \tau}\left(1-n_{0}\right)\right| \Psi>
\end{aligned}
$$

where the effective Hamiltonian $\hat{H}_{p}$ is defined in Eq. 3.12 and the fermion projection operators $n_{0}=\psi_{0}^{\dagger} \psi_{0}$, $\left(1-n_{0}\right)=\psi_{0} \psi_{0}^{\dagger}$ force the spin at the origin of the chain to point upwards or downwards respectively. In the following, we will first carry out the calculation of the asymptotic behavior of the trace of the Green function matrix:

$$
G(p, \tau)=G_{\downarrow}(p, \tau)+G_{\uparrow}(p, \tau)=i<\Psi\left|e^{-i\left(\hat{H}_{p}-i \eta\right) \tau}\right| \Psi>
$$

which is expected to show all the singularities present in the two separate spin projections.

The long wavelength form of the Hamiltonian $\hat{H}_{p}$ has been already studied in the previous Section. $\hat{H}_{p}$ depends on the total momentum $p$ via a function $\epsilon_{h}\left(k_{h}\right)$ which represents the holon dispersion:

$$
\hat{H}_{p}=\epsilon_{h}(p+\hat{P})+\hat{H}_{\sigma}
$$

$\hat{H}_{\sigma}$ is a Luttinger liquid Hamiltonian characterized by suitable boundary conditions and commuting with the spinon momentum operator $\hat{P}$ defined on the chain of $L-1$ sites. Note that the Heisenberg state $\mid \Psi>$ instead refers to the $L$-site ring with periodic boundary conditions and therefore is not an exact eigenfunction of the spinon momentum operator $\hat{P}$. It is then convenient to express the state $\mid \Psi>$ as the sum of all its projections into subspaces of definite momentum $\hat{P}=Q$ and substitute this representation into the form (5.47): 


$$
\begin{aligned}
G(p, \tau) & =\frac{i}{\ell} \sum_{Q} Z(Q, \tau) e^{-i\left[\epsilon_{h}(p+Q)-i \eta\right] \tau} \\
Z(Q, \tau) & =\int_{0}^{\ell} e^{-i Q x} Z(x, \tau) d x \\
Z(x, \tau) & =<\Psi\left|e^{i\left(\hat{P} x-\hat{H}_{\sigma} \tau\right)}\right| \Psi>
\end{aligned}
$$

This is the general form of the one hole Green function which shows the effects of spin-charge decoupling on the dynamics of the hole. The spinon function $Z(Q, \tau)$ provides a generalization of the quantity $Z(Q)$ which characterizes the form of the single hole Green function in the $J \rightarrow 0$ limit [11,23]. Here, however, non trivial dynamics of the spins are induced by $\hat{H}_{\sigma}$ as long as $v_{s} \neq 0$.

Let us first discuss the calculation of the matrix element $Z(x, \tau)$ in the non interacting limit. In a free Fermi gas with arbitrary boundary conditions, the long wavelength Hamiltonian reduces to the sum of the kinetic terms for the two branches of right and left moving fermions. In the continuum limit the Hamiltonian and the momentum operator are given by:

$$
\begin{aligned}
H & =v_{s}\left(\hat{P}_{+}-\hat{P}_{-}\right)+\mathrm{const} \\
\hat{P} & =\hat{P}_{+}+\hat{P}_{-} \\
\hat{P}_{ \pm} & = \pm k_{F} \int_{0}^{\ell} d x \psi_{ \pm}^{\dagger}(x) \psi_{ \pm}(x)+i \int_{0}^{\ell} d x \psi_{ \pm}^{\dagger}(x) \partial_{x} \psi_{ \pm}(x)
\end{aligned}
$$

This particular form of the Hamiltonian shows a close relationship between energy and momentum operators which is clearly valid only in the non interacting limit. However, the more general interacting Luttinger liquid can be mapped to the free Fermi gas by the previously defined canonical transformation (5.40) leading to the conservation of the number of right and left moving fermions at long wavelength. As a consequence, all eigenstates would factorize in the product of two states, one for each branch and, via Eq. (5.50) also the function $Z(x, \tau)$ would split in the product of two terms $Z_{ \pm}^{\delta_{ \pm}}\left(x \mp v_{s} \tau\right)$ defined in each branch. However, in the microscopic model, defined on a lattice, higher order terms allow the excitation of a fermion from one branch to another (even for $K_{\sigma}=1$ ). Therefore, the function $Z(x, \tau)$ will contain contributions corresponding to all these excitations which we label by the number $m(-m)$ of extra fermions on the right (left) branch:

$$
\begin{aligned}
Z(x, \tau) & =\sum_{m=-\infty}^{\infty} e^{i\left(Q_{m} x-E_{m} \tau\right)} Z_{+}^{\left(\delta_{+}, m\right)}\left(x-v_{s} \tau\right) Z_{-}^{\left(\delta_{-},-m\right)}\left(x+v_{s} \tau\right) \\
& =\sum_{m=-\infty}^{\infty} e^{i\left(Q_{m} x-E_{m} \tau\right)}<e^{i\left(\hat{P}_{+}-Q_{+}(m)\right)\left(x-v_{s} \tau\right)}>_{+}<e^{i\left(\hat{P}_{-}-Q_{-}(-m)\right)\left(x+v_{s} \tau\right)}>_{-}
\end{aligned}
$$

where the average labeled by $+(-)$ is taken on the ground state of the right (left) branch in the undoped system and the intermediate states are constrained to have $m(-m)$ additional particles on the right (left) branch. $Q_{m}=$ $Q_{+}(m)+Q_{-}(-m)$ and $E_{m}=v_{s}\left(Q_{+}(m)-Q_{-}(-m)\right)$ represent the reference momentum and energy of the intermediate states with $m$-particle excitations which are explicitly given in terms of the Fermi momentum of the spinons by $Q_{+}(m)=k_{F}(m+1)$ and $Q_{-}(-m)=-k_{F}(-m)$. The functions $Z_{ \pm}^{\left(\delta_{ \pm}, \pm m\right)}(x, \tau)$ introduced in Eq. (5.52) are well defined also in the long wavelength limit where the spinon Hamiltonian $\hat{H}_{\sigma}$ can be written in the bosonized form (5.8). As discussed in Sections $\mathrm{VA}$ and $\mathrm{VB}$ the quantum number $m$ which characterizes the inter-branch excitations appears in the low energy Hamiltonian only through the quantity $\Pi^{*}$ and can be absorbed in the definition of the phase shifts: $\bar{\delta}_{+}=\delta_{+}+m, \bar{\delta}_{-}=\delta_{-}+m$ (see Eqs. 5.10, 5.20, 5.21). The calculation of $Z(x, t)$ then reduces to the evaluation of the contribution appropriate for each branch with arbitrary phase shift $\bar{\delta}_{ \pm}$. The technical details are discussed in appendix B, here we just report the asymptotic behavior in the thermodynamic limit (B.22):

$$
Z_{ \pm}^{\bar{\delta}}(x) \propto(\mp i x)^{-\bar{\delta}^{2}}
$$

By substituting this result into Eq. (5.50), the spectral weight $A(p, \omega)=\frac{1}{\pi} \operatorname{Im} G(p, \omega)$ is written in terms of the $(Q, \omega)$ Fourier transform of $Z$ :

$$
A(p, \omega)=\int \frac{d Q}{2 \pi} Z\left(Q, \omega-\epsilon_{h}(p+Q)\right)
$$




$$
\begin{aligned}
Z(Q, \omega)= & \int_{-\infty}^{\infty} \int_{-\infty}^{\infty} d \tau d x e^{-i(x Q-\omega \tau)} Z(x, \tau) \\
= & \sum_{m} C_{m} \Theta\left[\omega+v_{s}\left(Q-Q_{m}\right)\right] \Theta\left[\omega-v_{s}\left(Q-Q_{m}\right)\right] \\
& v_{s}^{1-2 X_{m}}\left[\omega+v_{s}\left(Q-Q_{m}\right)\right]^{\bar{\delta}_{+}^{2}-1}\left[\omega-v_{s}\left(Q-Q_{m}\right)\right]^{\bar{\delta}_{-}^{2}-1}
\end{aligned}
$$

In the Fourier transform of $Z(x, \tau)$ only the singular contributions have been included. $\Theta(x)$ represents the step function whose presence is a direct consequence of the Fourier positivity (negativity) of the function $Z_{+}\left(Z_{-}\right)$, as discussed in appendix B. This property has a simple physical meaning: The particle hole excitations within the right branch of a Luttinger model can only increase the total momentum with respect to the ground state, while the excitations on the left branch can only decrease the total momentum of the system. Therefore the spinon spectral function at $Q>0$ has contributions coming only from excitations in the right branch and vice versa. The constants $C_{m}$, which only depend on the phase shifts $\delta_{+}$and $\delta_{-}$in a symmetric fashion, can be explicitly calculated in the free Fermi gas with skew boundary conditions as shown in Eq. (B.24) of appendix B. However, we expect that in the interacting models these coefficients will be renormalized in a non universal way which depends on the physical cut-off present in the microscopic model.

By inserting Eq. (5.55) into Eq. (5.54), the contribution coming from each excited state $m$ gives a divergence in the spectral function as long as $\bar{\delta}_{+}^{2}+\delta_{-}^{2}<1$. The singularities are located along the lines

$$
\Delta \omega_{m}=\omega-\epsilon_{h}\left(p+Q_{m}\right)=0
$$

in the $(p, \omega)$ plane and show the asymptotic behavior:

$$
A(p, \omega) \propto\left|\Delta \omega_{m}\right|^{2 X_{m}-1}
$$

where $X_{m}=\frac{1}{2}\left(\bar{\delta}_{+}^{2}+\bar{\delta}_{-}^{2}\right)$. In general, we expect that the phase shifts $\delta_{ \pm}$which characterize the singularities of the spectral function depend on the holon momentum $k_{h}$. Therefore, $A(p, \omega)$ will show singularities along the lines $\omega=\epsilon_{h}\left(p+Q_{m}\right)$ with momentum dependent exponents $X_{m}$.

Another unexpected prediction of the present formalism concerns the behavior of the spectral function in a neighborhood of the singularity: By a direct evaluation of the prefactor we find different results according to whether the charge velocity is larger or smaller than the spin velocity. In fact, if $\left|v_{c}\right| \leq v_{s}$, there are divergences only for $\omega \geq \epsilon_{h}\left(p+Q_{m}\right)$ while the spectral weight vanishes on the other side of the singularity line $\Delta \omega_{m}=0$ as there are no states with lower energy contributing to the spectral weight:

$$
A(p, \omega) \propto \frac{\left|\Delta \omega_{m}\right|^{2 X_{m}-1}}{\left(v_{s}+v_{c}\right)^{\bar{\delta}_{+}^{2}}\left(v_{s}-v_{c}\right)^{\bar{\delta}_{-}^{2}}}
$$

Instead if $\left|v_{c}\right|>v_{s}$ the spectral function diverges on both sides of the singularity with the same exponent $2 X_{m}-1$ but different prefactor:

$$
A(p, \omega) \propto \frac{\pi}{\sin \left(\pi \bar{\delta}_{\mp}^{2}\right)} \frac{\left|\Delta \omega_{m}\right|^{2 X_{m}-1}}{\left|v_{s}+v_{c}\right|^{\bar{\delta}_{+}^{2}}\left|v_{s}-v_{c}\right|^{\bar{\delta}_{-}^{2}}}
$$

where the upper sign and the lower one refer to $\pm v_{c} \Delta \omega>0$, respectively. The amplitude ratio can be evaluated from Eq. (5.59):

$$
\frac{A(\Delta \omega)}{A(-\Delta \omega)}=\frac{\sin \left(\pi \delta_{ \pm}^{2}\right)}{\sin \left(\pi \delta_{\mp}^{2}\right)}
$$

This simple expression, which shows the asymmetry of the spectral weight above and below the singularity, is strictly valid only for the Luttinger model, but is also expected to be qualitatively correct in more general cases. In fact this feature originates from the different energy spectrum of the excitations on the left and right branches, which is a common property of one dimensional correlated systems. Notice also that the prefactors in (5.58, 5.59) are strongly enhanced close to the instability which sets in when the charge velocity equals the spin velocity.

When we switch on interactions among spinons the above expressions (5.54, 5.55, 5.58, 5.59) remain formally unchanged if the phase shifts are suitably renormalized by $K_{\sigma}$ analogously to Eq. (5.41) where $\delta_{ \pm}^{\text {eff }} \rightarrow \bar{\delta}_{ \pm}$and $\delta \rightarrow(\delta+m)$. As a consequence, the exponent of the leading singularity (i.e. that corresponding to $m=0$ ) $X_{0}$ exactly reproduces the exponent already obtained in the calculation of the overlap $\zeta$ (5.42). Moreover, the full set of 
exponents $X_{m}$ agrees with the term proportional to $2 \pi v_{s} / \ell$ in the finite size correction to the energy of the model (5.35) for the particular choice of the quantum number $n=0$. The restriction $n=0$ is due to the fact that the intermediate states appearing in the rotational invariant Green function (5.47) do not have definite spin and then, at fixed $m$, the leading singularity is related to the smallest phase shift which corresponds to $n=0$. It is however clear how to generalize these expressions for the calculation of the spin up (or spin down) Green function: The sum over intermediate states in Eq. (5.49) has to be restricted to the states with the correct spin projection. Within bosonization it means states with the appropriate value of the quantum number $n$ which is in fact related to the total spin of the one hole intermediate state through Eqs. (5.9.5.10). This procedure in fact reproduces the full set of critical exponents $X_{m, n}$ which appear in Eq. (5.35).

Finally, if we want to evaluate the singularities in the hole density of states we just have to integrate the leading momentum dependence of the spectral function $(5.57)$ :

$$
N(\omega)=\int \frac{d p}{2 \pi} A(p, \omega) \propto \sum_{m} c_{m} \omega^{2 X_{m}(0)-1 / 2}+\text { const }
$$

where $c_{m}$ are finite amplitudes, $\omega$ is measured from the bottom of the band and the exponent $X_{m}(0)$ coincides with the previously introduced critical exponent $X_{m}$ evaluated at the bottom of the holon band i.e. at a total momentum $p$ such that $k_{h}=p+Q_{m}$ sits at the minimum of $\epsilon_{h}\left(k_{h}\right)$. In fact this region of integration in momentum space gives rise to the leading singularity in the density of states. Note that a divergence in the density of states occurs only if $X_{m}(0)<1 / 4$ for some $m$.

\section{SINGLE HOLE IN THE XY MODEL}

Here we analyze in some detail the dynamical properties of a single hole in the $X Y$ model on the basis of the Hamiltonian already introduced in Section IV] in the limit of small hole mass: $J^{\prime}=J<<t$. The aim of this study is to check, in a simple model, all the general features of hole motion already discussed in Section $\mathrm{V}$ and to carry out the quantitative evaluation of exponents and amplitudes for this system.

The Hamiltonian of a hole of momentum $p$ in the $t-J_{X Y}$ model (4.1) has been previously derived in some detail and reads:

$$
\hat{H}_{p}=t \exp \left[i p+i \sum_{k} k \psi_{k}^{\dagger} \psi_{k}\right]+\text { h.c. }-\frac{J}{2} \sum_{i=1}^{\ell-1}\left[\psi_{i}^{\dagger} \psi_{i+1}+h . c .\right]
$$

Here we consider a spin down hole of momentum $p$ in a chain with even number of sites $L$ and odd number of up spins (i.e. an odd number of fermions in the representation of Eq. (6.1)) $N=2 \nu+1$ corresponding to a $z$-axis magnetization $\mu=-\frac{1}{2}+\frac{N}{L}$. According to the discussion in Section III, the appropriate boundary conditions of the fermionic problem are therefore antiperiodic, $N+L$ being odd, and the quantization rule for the momenta is

$$
k_{j}=\frac{2 \pi}{\ell}\left(j+\frac{1}{2}\right)
$$

with $\ell=L-1$ as usual. The states which diagonalize the hole kinetic term are states of given spinon momentum $Q$. In the $J \rightarrow 0$ limit the spectrum of the Hamiltonian (6.1) can be obtained by diagonalizing the magnetic term in the subspace of fixed spinon momentum $Q$. This procedure gives rise to spinon Slater determinants of plane waves. The ground state is doubly degenerate and corresponds to a set of $N$ occupied orbitals of momenta centered around $k=0$, while low energy excitations can be obtained either by changing the number of fermions (i.e. the magnetization) $N \rightarrow N+n$ or by moving $m$ fermions from the left to the right branch of the Fermi surface. The energy of these excited states can be easily calculated by taking the expectation value of the magnetic term on the appropriate spinon Slater determinant. Energy and momentum are therefore given by:

$$
\begin{aligned}
E_{p} & =2 t \cos (p+Q)-J \frac{\ell-1}{\ell} \sum_{j=-\nu-n+m}^{\nu+n+m} \cos \left[\frac{2 \pi}{\ell}\left(j+\frac{1}{2}\right)\right] \\
Q & =\sum_{j=-\nu-n+m}^{\nu+n+m} \frac{2 \pi}{\ell}\left(j+\frac{1}{2}\right)
\end{aligned}
$$

These expressions, being based on perturbation theory in $J<<t$, are exact to $O(J / t)$ and to $O(1)$ respectively. By carrying out the summations and expanding up to $O(1 / \ell)$ at fixed $\rho=N / L$, we find: 


$$
\begin{aligned}
E_{p}= & -J(\ell-1) \frac{\sin \pi \rho}{\pi}-J \cos \pi \rho(n+\rho)+2 t \cos (p+Q)+ \\
& \frac{2 \pi J \sin \pi \rho}{\ell}\left[\left(m+\frac{1}{2}\right)^{2}+\frac{1}{4}(n+\rho)^{2}\right]-J \sin \pi \rho \frac{\pi}{6 \ell}+\frac{J \cos \pi \rho}{\ell}(n+\rho) \\
Q= & 2 \pi \rho\left(m+\frac{1}{2}\right)+\frac{2 \pi}{\ell}(n+\rho)\left(m+\frac{1}{2}\right)
\end{aligned}
$$

In the thermodynamic limit, besides the extensive magnetic contribution, the energy of the state depends on the holon momentum $k_{h}=p+Q$ through the form of the holon band $\epsilon_{h}(k)=2 t \cos k_{h}$. The spinon momentum is instead given by $Q=2 k_{F}\left(m+\frac{1}{2}\right)$ when we recognize that the spinon Fermi momentum is just $k_{F}=\pi \rho$. The $O(1 / \ell)$ size corrections to momentum and energy can be also compared to the general expressions (5.26.5.35) if we recall that the spinon velocity is $v_{s}=J \sin k_{F}=J \sin \pi \rho$ and $K_{\sigma}=1$ in the $X Y$ model. Equations (6.5) and (6.6) correctly reproduce the predicted structure of the size corrections showing that the hole dynamics is described by an underlying conformal field theory. This comparison allows to determine, to lowest order in $J / t$, the values appropriate for the $t-J_{X Y}$ model of the non universal parameters entering Eq. (5.35):

$$
\delta=\frac{1}{2}\left(\delta_{+}+\delta_{-}\right)=\frac{1}{2} \quad \gamma=\left(\delta_{+}-\delta_{-}\right)=\rho
$$

for the phase shifts and

$$
\omega_{+}=\frac{\ell}{2 \pi}\left(k_{F}^{+}+k_{F}^{-}\right)=0 \quad \omega_{-}=\frac{\ell}{2 \pi}\left(k_{F}^{+}-k_{F}^{-}\right)=O(J)
$$

for the shifts of the right and left Fermi wavevectors.

According to Section $\mathrm{VG}$ the phase shifts (6.7) completely determine the singularities of the Green function. As a check, let us explicitly evaluate the overlap $\zeta(5.36)$ in this model. In fermionic representation this amounts to calculate the overlap between the ground state of Hamiltonian (6.1) and the ground state of the $X Y$ model on a $L$-site ring with the same number of fermions $N=2 \nu+1$. In fermion representation the latter state is just a Slater determinant of plane waves with momentum quantization corresponding to periodic boundary conditions on a $L$-site ring:

$$
q_{j}=\frac{2 \pi}{L} j
$$

Therefore, $\zeta$ is simply the overlap of two Slater determinants of plane waves with different quantization rule (6.2),6.9). Such an overlap can be calculated as the determinant of the $N \times N$ matrix of the overlaps of the two sets of plane waves defined on the squeezed chain:

$$
\begin{aligned}
\zeta_{p, \downarrow} & =\operatorname{det} H \\
H_{r s}=<q_{r} \mid k_{s}> & =i \frac{e^{i k_{s} / 2}}{\sqrt{L(L-1)}} \frac{\cos \left[q_{r} / 2\right]}{\sin \left[\left(k_{s}-q_{r}\right) / 2\right]}
\end{aligned}
$$

with $r$ and $s$ belonging to the interval $[-\nu, \nu]$. Notice that the quantization rule of the wavevectors $k_{j}$ can be naturally interpreted in terms of a momentum dependent phase shift:

$$
k_{j} \sim \frac{2 \pi}{L}\left(j+\frac{1}{2}+\frac{j}{L}\right)
$$

Near the Fermi points the phase shifts are then given by: $\delta_{ \pm}=\frac{1 \pm \rho}{2}$ in agreement with their determination based on the structure of the finite size corrections to the energy (6.7). The known treatment [21] of the orthogonality catastrophe problem thus gives the critical exponent in terms of the phase shift at the Fermi points: $2 X_{0}=\delta_{+}^{2}+\delta_{-}^{2}$ which agrees with the expression (5.39) derived in Section $\mathrm{VG}$. This result can be also checked numerically by evaluating the determinant (6.10) for fairly large system sizes. The size scaling of $\ln \zeta$ as a function of $\ln N$ is shown in Fig.4 for two magnetizations $(\mu=0$ and $\mu=1 / 4)$ corresponding to the densities of $\operatorname{spin}$ up $\rho=1 / 2$ and $\rho=3 / 4$. The analytical value of the exponent $X_{0}$ is also shown in figure. The exponent for the $\rho=1 / 2$ case also agrees with an independent calculation by Penc et al. [24].

The next task is the evaluation of the spin down Green function (5.44) which, via Galileo transformation, takes the form (5.46). In the $J \rightarrow 0$ limit, the energy levels can be written as the sum of a holon part $\epsilon_{h}(p+Q)=2 t \cos (p+Q)$, 
which just depends on the spinon momentum $Q$, and a spinon term which is eigenstate of the $X Y$ Hamiltonian on the squeezed chain. Therefore, at fixed total momentum $p$, the Green function reads

$$
G(p, \tau)=\frac{i}{\ell} \sum_{Q} \sum_{R} e^{-i Q R} e^{-i \epsilon_{h}(p+Q) \tau} Z(R, \tau)
$$

where the spinon term, implicitly depending on the total momentum $p$, is

$$
Z(R, \tau)=<\Psi\left|e^{-i \hat{H}_{\sigma} \tau} T_{\ell}^{R}\left(1-n_{0}\right)\right| \Psi>
$$

Here $\hat{H}_{\sigma}$ and $T_{\ell}$ respectively represent the $X Y$ spinon Hamiltonian and the translation operator of one lattice spacing on the squeezed ring of $\ell=L-1$ sites (origin excluded) while $\mid \Psi>$ is the ground state of the $X Y$ model on the $L$-site ring. Notice that, for the special case of the $X Y$ model at $J \rightarrow 0$, the decoupling of the Green function in holon and spinon factors

$$
G(R, \tau)=Z(-R, \tau) G_{h}(R, \tau)=Z(-R, \tau) i \int_{-\pi}^{\pi} \frac{d k}{2 \pi} e^{i k R} e^{-i \epsilon_{h}(k) \tau}
$$

is exact at all distances while, in general, we expect this decoupling is valid only at low energy and long wavelength, i.e. for $R, \tau \gg 1$.

The evaluation of the matrix element in Eq. (6.14) can be performed because in the spinon representation the two sates are Slater determinants (without phase shifts) and the unitary operator acting on them is a one body operator. The projection operator $\left(1-n_{0}\right)$ implies that the origin is an empty site which amounts to exclude the origin in the evaluation of the overlap matrix. Therefore, $Z(R, \tau)=\operatorname{det} A(R, \tau)$ where the overlap matrix (of linear dimension $2 \nu+1)$ can be easily calculated by inserting a complete set of orbitals which do not place particles in the origin. A useful choice is the set of eigenstates of the spinon Hamiltonian $\hat{H}_{\sigma}$ corresponding to an odd number of spinons i.e. with momenta (6.2). The resulting form of the overlap matrix is:

$$
A_{r s}(R, \tau)=\frac{\cos \left(q_{r} / 2\right) \cos \left(q_{s} / 2\right)}{L(L-1)} \sum_{j=-L / 2+1}^{L / 2-1} \frac{e^{i\left(k_{j} R+\tau J \cos k_{j}\right)}}{\sin \left[\left(k_{j}-q_{r}\right) / 2\right] \sin \left[\left(k_{j}-q_{s}\right) / 2\right]}
$$

The sum can be performed analytically only in the static limit $\tau=0$ where the numerical computation of $Z(R, 0)$ and of its Fourier transform $Z(Q)$ can be pushed to fairly large system sizes. A comparison between two different sizes at fixed magnetization $(\mu=0$ and $\mu=1 / 4)$ is shown in Fig. 5a where we can see that $Z(Q)$ does not vanish outside the "spinon Fermi surface" even if it is strongly suppressed. The singularity at the Fermi momentum $k_{F}=\pi \rho$ appears to be present on both sides of the spinon Fermi surface. A logarithmic plot of the singularity of $Z(Q)$ when $Q$ approaches $k_{F}$ is shown in Fig. 5b where it is compared to the analytical value of Eqs. (6.7, B.24). The ratio of the two amplitudes on both sides of the singularity is in rather good quantitative agreement with expression (5.60).

Now we examine in some detail the asymptotic form of the dynamical spinon Green function of the $t-J_{X Y}$ model (6.14). As noted before, the exact calculation of $Z(R, \tau)$ in a finite system reduces to the evaluation of the determinant of the matrix $A_{r s}(R, \tau)$ defined in Eq. (6.16). The exact calculation can be performed only numerically and the direct interpretation of the data is obscured by finite size effects. However, we can address the problem of the long distance and long time behavior of $Z(R, \tau)$ by performing the asymptotic expansion of the matrix elements $A_{r s}$ themselves. This expansion can be carried out rather easily in the low density limit where, according to expectations of conformal field theory, it should be characterized by phase shifts $\delta_{+}=\delta_{-}=1 / 2$ (6.7). The details are reported in appendix $\mathrm{Q}$.

According to bosonization, the spinon Green function should behave as (5.53)

$$
Z(R, \tau) \propto \frac{e^{i k_{F} R}}{\left(R-v_{s} \tau\right)^{1 / 4}\left(R+v_{s} \tau\right)^{1 / 4}}+\frac{e^{-i k_{F} R}}{\left(R-v_{s} \tau\right)^{1 / 4}\left(R+v_{s} \tau\right)^{1 / 4}}
$$

where $v_{s}=J k_{F}$ and this expression is valid for $\left|v_{s} \tau\right|<R$. From this analysis we expect that the function $Z(R, \tau)$ at long wavelength behaves as

$$
Z_{0}(R, \tau) \propto \frac{\cos \left(k_{F} R\right)}{\left(R-v_{s} \tau\right)^{1 / 4}\left(R+v_{s} \tau\right)^{1 / 4}}
$$

In Fig. 6 we plot the numerically evaluated ratio $|\operatorname{det} A(R, \tau)| / Z_{0}(R \tau)$ as a function of $R-v_{s} \tau$ for $N=100$ and $\mu=-1 / 4$ which belongs to the low density regime. This ratio has no oscillations (meaning that the phase factor has 
been correctly determined) and it is approximately constant over a wide range of values of $R-v_{s} \tau$. Clearly the region $R \sim v_{s} \tau$ cannot be well represented by the bosonized form which would predict a spurious divergence in the Green function which instead is bound to have modulus less than unity.

The analysis of this Section shows in a simple example that all the features of hole propagation in a magnetic background derived with field theoretical formalism are contained in such a microscopic model. This detailed calculation supports the assumptions introduced in our general study of the long wavelength hole dynamics. More interesting systems can now be investigated.

\section{BETHE ANSATZ MODELS}

In this Section we consider two Bethe ansatz solvable models, the repulsive Hubbard model [6] and the $t-J$ model at $J=2 t$ [26], where the finite size corrections to the energy can be found analytically, leading to a formal expression for the critical exponent which appears in the one hole Green function. We analyze both models at arbitrary magnetization so that our results can be extended to the attractive Hubbard model via the well known canonical transformation.

The calculation of the finite size corrections to the ground state energy closely follows the original derivation by Woynarovich for the Hubbard model at finite density [27]. Here we consider a chain of $L$ sites and $L-1$ electrons, among which $N_{\downarrow}$ have spin down. The Bethe ansatz solution is characterized by two sets of rapidities: For the Hubbard case we have $N_{s}=N_{\downarrow}$ rapidities $\lambda_{\alpha}$ and $N_{c}=L-1$ rapidities $k_{j}$, while for the $t-J$ model (in Sutherland representation) we have $N_{s}=N_{\downarrow}+1$ rapidities $v_{\alpha}$ and $N_{c}=1$ rapidity $w_{0}$. These rapidities are related to the quantum numbers $J_{\alpha}$ and $I_{j}$ respectively through the Bethe ansatz equations. In the ground state, the two sets of quantum numbers define two compact distributions bounded by $J^{ \pm}$and $I^{ \pm}$respectively. The explicit expressions of $J^{ \pm}$and $I^{ \pm}$are :

$$
\begin{array}{ll}
J^{+}-J^{-}=N_{s} & J^{+}+J^{-}=2 D_{s} \\
I^{+}-I^{-}=N_{c} & I^{+}+I^{-}=2 D_{c}
\end{array}
$$

where $N_{s}$ and $N_{c}$ have been previously defined while the "centers" of the distributions $D_{s}$ and $D_{c}$ specify the spin and charge state respectively. In the ground state, at fixed total momentum $p, D_{s}$ is the smallest integer (or half integer) compatible with the quantization rules for $J_{\alpha}$. Instead, $D_{c}=I_{h}+L / 2$ for the Hubbard model and $D_{c}=I_{h}$ for the $t-J$, where $I_{h}$ defines the position of the hole in the distribution of charge rapidities and is related to the momentum of the holon. Following Woynarovich, we define the four "densities" as the $L \rightarrow \infty$ limit of $\nu_{c(s)}=N_{c(s)} / L$ and $\delta_{c(s)}=D_{c(s)} / L$, so that $D_{c(s)}-\delta_{c(s)} L$ and $N_{c(s)}-\nu_{c(s)}$ are finite for $L \rightarrow \infty$. If a hole of momentum $p$ and spin down is created, the total momentum of the state is given by:

$$
-p=-p_{0}+\frac{2 \pi}{L}\left[\left(N_{s}-\nu_{s} L\right)\left(D_{s}-\delta_{s} L\right)+\left(N_{c}-\nu_{c} L\right)\left(D_{c}-\delta_{c} L\right)\right]
$$

where $-p_{0}=2 \pi\left(D_{c} N_{c}+D_{s} N_{s}\right) / L$ is the momentum in the thermodynamic limit. Analogously, the size corrections to the ground state energy $E$ for the single hole problem can be expressed in terms of the above defined quantities, the charge (spin) velocity $v_{c(s)}$, the elements of the dressed charge matrix $\xi_{i j}$ and of the additional matrix $Z_{i j}$ as:

$$
\begin{aligned}
& L\left(E-L \epsilon_{\infty}\right)=-\frac{\pi}{6} v_{s}+2 \pi v_{c} X_{c}+2 \pi v_{s} X \\
& X_{c}=-\left(N_{c}-\nu_{c} L\right)\left[\left(D_{c}-\delta_{c} L\right)+\xi_{12}\left(D_{s}-\delta_{s} L\right)-\Delta Z_{21} \frac{\left(N_{s}-\nu_{s} L\right)-\xi_{12}\left(N_{c}-\nu_{c} L\right)}{2 \xi_{22}}\right] \\
& X=\xi_{22}^{2}\left[\left(D_{s}-\delta_{s} L\right)-Z_{12}\left(N_{c}-\nu_{c} L\right)\right]^{2}+\left[\frac{\left(N_{s}-\nu_{s} L\right)-\xi_{12}\left(N_{c}-\nu_{c} L\right)}{2 \xi_{22}}\right]^{2}
\end{aligned}
$$

where $\epsilon_{\infty}$ is the ground state energy per site of the model at half filling and $N_{c}-\nu_{c} L=-1$ for the Hubbard case (where $N_{c}=L-1$ ) and $N_{c}-\nu_{c} L=1$ for the $t-J$ model (where $N_{c}=1$ ). The diagonal element of the dressed charge matrix is simply related to the correlation exponent of the Heisenberg model introduced in Section $\mathrm{V}$ by the well known expression $K_{\sigma}=\xi_{22}^{2}$ [16]. This general formula is valid both in the Hubbard and in the $t-J$ model. The basic steps for the formal derivation of Eq. (7.3) together with the precise definitions of the quantities appearing in it are reported in Appendix D. Here we only stress the decoupling of the charge and the spin terms in the finite size corrections, in agreement with our starting assumptions: The long wavelength Hamiltonian is the sum of a charge part which does not give any singularity in the correlation functions and a spin part which instead gives rise to critical 
exponents. Most importantly, this rather complicate, exact, expression $(7.3)$ perfectly matches the predictions of the bosonization method (5.35).

From Eq. (7.2) we find that the size scaling of the total momentum is characterized by the amplitude (5.33) $\alpha=-\left(N_{s}-\nu_{s} L\right)\left(D_{s}-\delta_{s} L\right)-\left(N_{c}-\nu_{c} L\right)\left(D_{c}-\delta_{c} L\right)$ while from the magnetic contribution in Eq. (7.3) we easily identify the quantum numbers $(m, n)$ and the phase shifts $(\delta, \gamma)$. The other parameters appearing in Eq. (5.35) then follow from the charge part of Eq. (7.3) leading to:

$$
\begin{aligned}
\alpha & =-\left(N_{s}-\nu_{s} L\right)\left(D_{s}-\delta_{s} L\right)-\left(N_{c}-\nu_{c} L\right)\left(D_{c}-\delta_{c} L\right) \\
m & =D_{s}-\frac{1}{2} \\
n & =\left(N_{s}-\nu_{s} L\right) \\
\delta & =\frac{1}{2}-Z_{12}\left(N_{c}-\nu_{c} L\right) \\
\gamma & =-\xi_{12}\left(N_{c}-\nu_{c} L\right) \\
\omega_{+} & =0 \\
\omega_{-} & =\frac{\Delta Z_{21}\left(N_{c}-\nu_{c} L\right)}{\xi_{22}}+2 Z_{12}\left(N_{c}-\nu_{c} L\right)
\end{aligned}
$$

where we used that in the ground state $D_{s}=1 / 2$ and then $\delta_{s}=0$. Note the vanishing of $\omega_{+}$which occurs in all the models we have examined and is probably related to a sort of Luttinger theorem which forces the volume of the spinon Fermi surface to be unaffected by interactions. This exact correspondence between bosonization and Bethe ansatz demonstrates the validity of our approach in the Hubbard and $t-J$ models and allows for the analytical determination of the phase shifts $\delta_{ \pm}$governing the singularities of the one hole Green function.

In general, the coefficients appearing in Eq. (7.3) are non universal quantities which depend on the coupling constants of the model as well as the average magnetization per site $\mu$ and the holon momentum. Therefore, we expect that the exponent $X$ is a function of all the parameters which define the hole Hamiltonian, including the total momentum $p$. However, some special but important exception must be mentioned. At zero magnetic field the spin Hamiltonian possesses the additional $S U(2)$ symmetry both in the Hubbard and $t-J$ model. At this particular point, the exponent $X$ is universal. In fact, the elements of the matrices $\xi$ and $Z$ acquire analytic values independent of the total momentum of the state and of the coupling constants: $\xi_{22}=1 / \sqrt{2}, \xi_{12}=1 / 2$ and $Z_{12}=0$. Another simple case is the $U \rightarrow \infty$ limit of the Hubbard model where $Z_{12}=0$ and the dressed charge matrix can be expressed in terms of the magnetization per site $\mu=\left(N_{\uparrow}-N_{\downarrow}\right) / 2 L: \xi_{12}=1 / 2-\mu$, while $K_{\sigma}=\xi_{22}^{2}$ as a function of $\mu$ is shown in Fig. 1 of Ref. [12]. Also in this limit the exponents do not depend on the total momentum of the state. Finally, when the hole sits at the bottom of the band, i.e. if we are at the one hole ground state, the equations simplify because the holon momentum is always $k_{h}=\pi$ and so $v_{c}=0$. Again $Z_{12}=0$ and our expression for the finite size corrections coincides with the zero doping limit of the known form valid at finite density. This proves the continuity between the physics of the single hole problem and that of finite doping in $1 \mathrm{D}$.

At arbitrary magnetization and momentum no analytical expression for the phase shifts is available. However, the integral equations reported in the Appendix D can be solved numerically. Few examples are reported in Fig. 7 where we show the critical exponents $X_{m}(5.42$ ) as a function of the momentum for several magnetizations in the Hubbard model. The loci $\omega=\epsilon_{h}\left(p+Q_{m}\right)$ in the $(p, \omega)$ plane where the occurrence of the divergence is predicted by our theory are instead shown in Fig. 8 for a couple of choices of the parameters. The presence of $2 k_{F}$ zero energy excitations in the spinon spectrum gives rise to a remarkable symmetry property of these curves: At a given energy $\omega$, if a singularity occurs at momentum $p$ it will also show up, with possibly different exponent, at momentum $-p+2 k_{F}$.

As a check on the theory of Section $\mathrm{V}$ we have numerically evaluated the overlap of Eq. (5.36) by Lanczos diagonalization in the $t-J$ model at $J=2 t$ in chains up to 32 sites at magnetization $\mu= \pm 1 / 4$. The results are well fitted by a power law behavior in $L$ (5.38) with an exponent $X$ which clearly depends on the momentum of the hole, in agreement with the bosonization analysis. A comparison between the numerically determined exponent and the prediction of Eq. (7.3) is shown in Fig. 9 (a). An analogous computation has also been performed in the $t-J$ model at the generic non integrable point $J=t$ and $\mu=1 / 4$. The numerical results are also shown in figure 10 (b) but in this case the comparison with the analytical predictions based on the Bethe Ansatz solution is not available. However, a quite similar momentum dependence of the overlap exponent $X$ emerges, showing that the above features are not special to the exactly integrable points. 


\section{THE SHAPE OF THE SPECTRAL FUNCTION}

In this Section we finally discuss the global shape of the spectral function of one hole in a correlated background. In fact, the bosonization method developed in Section $\square$ only concerns the long wavelength, critical properties of the Green function and gives no information on its short wavelength features. The exact calculation of a Green function in an interacting system, at all lengthscales, has been achieved only recently [29] by use of extremely sophisticated methods for the Calogero-Sutherland model [30] while no results are available in other interacting systems. Our task is twofold: to understand the physical nature of the low lying excitations which contribute to the spectral function and to develop a useful numerical method for the approximate determination of the spectral function $A(p, \omega)$ which can be applied to generic one dimensional models. The purpose is to overcome the severe finite size effects present in Lanczos diagonalizations 31, 32] without resorting to the delicate extrapolations of simulation data 33] necessary for the computation of dynamical correlation functions.

Here, we formulate a simple approximation for the one hole Green function which captures most of the features of the exact result and can be usefully applied to interesting correlated models, in one dimension, like Hubbard. A similar approximation has been discussed by Penc et al. 23] in the strong coupling limit of the model. For clarity, we introduce the method in the framework of the previously discussed $t-J_{X Y}$ Hamiltonian in the $J \rightarrow 0$ limit (see Section VI leaving the study of the Hubbard model as a final example.

The starting, exact, expression for the $1 \mathrm{D}$ Green function of the $t-J_{X Y}$ model is Eq. (6.13) where the spinon function $Z(R, \tau)$ is defined by Eq. 6.14). The spinon term $Z$ in fact contains all the interesting correlation effects as previously pointed out. However, its direct evaluation proved rather hard even in the simple case of the $X Y$ model where the exact ground state is a Slater determinant, while the more realistic case of a Hubbard model cannot be tackled by these methods. As an approximate way to evaluate the matrix element (6.14) we can assume that the most relevant contribution to the intermediate states comes from the single spinon states, i.e. from the exact eigenstates of $\hat{H}_{\sigma}$ in the squeezed chain with only one spinon excitation. In fact, being $H_{\sigma}$ an antiferromagnetic Hamiltonian defined in an odd chain with periodic boundary conditions, it gives rise to a frustrated problem. Then, its ground state contains a free spinon and it is rather natural to assume that a set of low energy states can be built by giving a finite momentum $Q$ to such a "quasiparticle". These single spinon states $\mid Q>$ can be therefore labeled by the momentum $Q$, which lies outside the spinon Fermi surface, and have energy $\epsilon_{\sigma}(Q)$ given by the spinon band: $\epsilon_{\sigma}(Q)=J \cos Q$ for the $X Y$ model, where $|Q|>k_{F}$ and $k_{F}=\pi \rho$ is the spinon Fermi momentum. The single spinon approximation to the function $Z(R, \tau)$ therefore reads:

$$
Z(R, \tau) \sim \frac{1}{\ell} \sum_{|Q|>k_{F}} e^{-i \epsilon_{\sigma}(Q) \tau} e^{i Q R}|<Q|\left(1-n_{0}\right)|\Psi>|^{2}=\frac{1}{\ell} \sum_{|Q|>k_{F}} e^{-i \epsilon_{\sigma}(Q) \tau} e^{i Q R} Z(Q)
$$

where $\mid \Psi>$ is the ground state of the undoped model and we have introduced the spinon function $Z(Q)$

$$
Z(Q)=\sum_{R} e^{-i Q R}<\Psi\left|T_{\ell}^{R}\left(1-n_{0}\right)\right| \Psi>
$$

This approximation, therefore entirely resides in the assumption that only the single spinon intermediate states give a finite contribution to $Z(R, \tau)$. As a consequence, the time dependence of $Z(R, \tau)$ is greatly simplified but still not trivial due to the complex structure which can be present in $Z(Q)$.

The value of this approximation is that the time dependence of $Z(R, \tau)$ is given analytically in terms of the known spinon excitation spectrum of the model and that only a small $O(\ell)$ number of matrix elements is necessary for the evaluation of the full $Z(Q)$. The exact calculation in fact would require the insertion of a complete set of intermediate states leading to an exponentially large number of terms in the sum of Eq. (8.1). A consequence of the single spinon approximation is the presence of a sharp Fermi surface in the function $Z \bar{Q}$ ) which is in fact predicted to vanish identically for $|Q|<k_{F}$. This property is well satisfied in the quasiparticle weight for the $J \rightarrow 0$ limit of the $t-J$ model [11,23] even if both the numerical calculation of $Z(Q)$ in the $t-J_{X Y}$ model (see Fig. 5a) and its density matrix renormalization group evaluation in the strong coupling limit of the Hubbard model show that this is not an exact feature of one dimensional systems 34.

We now proceed to the evaluation of $Z(Q)$ in single spinon approximation for the exactly soluble $t-J_{X Y}$ model, in order to provide a check on the quality of this approximation. The completeness condition of the intermediate states which is obeyed by the exact quasiparticle weight reads:

$$
\sum_{Q} Z(Q)=\ell(1-\rho)
$$


In order to compute $Z(Q)$ in the $t-J_{X Y}$ model on a $L$-site ring and $2 \nu+1$ spinons, we first recall that the ground state of the $X Y$ model is a Slater determinant of $2 \nu+1$ plane waves with momentum quantization (6.9) and then the occupied orbitals are $\mid q_{s}>$ with $s \in[-\nu, \nu]$. The intermediate states are also Slater determinants of plane waves but with different quantization rule (6.2). If the spinon momentum is $Q_{r}=\frac{2 \pi}{\ell}\left(r+\frac{1}{2}\right)$, the first $2 \nu$ single particles intermediate states $\mid k_{j}>$ fill the spinon Fermi sea $j \in[-\nu, \nu-1]$ while the remaining spinon is placed outside this interval, at $j=r$. As noted before, the overlap between two Slater determinants is just the determinant of the matrix of the overlaps $B_{s j}=<q_{s} \mid k_{j}>$ and the quasiparticle weight $Z\left(Q_{r}\right)$ is the modulus square of such a determinant. The numerical evaluation of the completeness sum rule (8.3) in single spinon approximation is plotted in Fig. 10 for two magnetizations. The data show that the sum rule is violated in the thermodynamic limit and therefore the single spinon states do not represent a complete set of intermediate states as expected. However, Fig. 10 also shows that the breakdown of the sum rule is very small and shows up at considerably large system size. This approximation accurately reproduces the short wavelength properties of the model while fails in catching the long wavelength features (i.e. critical exponents and amplitudes) which we already discussed by use of bosonization methods in Section $\mathrm{V}$. Therefore, we expect that single spinon approximation can be successfully applied to the study of the global shape of the one hole spectral function in one dimensional models. As an example we now briefly discuss the case of the Hubbard model.

Analogously to the $t-J_{X Y}$ model, the Green function is expressed in terms of the dynamical quasiparticle weight by Eq. (6.13) which is approximately evaluated as in (8.1):

$$
G(p, \tau)=i \sum_{Q} e^{-i \epsilon_{h}(p+Q) \tau} e^{-i \epsilon_{\sigma}(Q) \tau}|<Q| c_{p, \downarrow}|\Psi>|^{2}
$$

The remaining problem is to compute the function $Z(Q)=\ell|<Q| c_{p, \downarrow}|\Psi>|^{2}$ for the Hubbard model. The numerical calculation can be carried out by Lanczos diagonalizations by exploiting the negligible size dependence of this quantity already verified in the previous examples. The ground state $|\Psi\rangle$ of the half filled Hubbard model at magnetization $\mu=-\frac{1}{2}+\frac{2 \nu+1}{L}$ can be numerically obtained in chains up to $L=16$ sites. The remaining problem is to select the one spinon states $\mid Q>$ which contribute to $Z(Q)$. The procedure we have adopted takes advantage of the continuity of the one spinon states between the weak and the strong coupling limit. At $U \rightarrow \infty$ the one spinon state $\mid Q>$ is in one to one correspondence with the ground state of momentum $Q$ of the Heisenberg chain in a $\ell=L-1$ site ring. This follows from the factorization property of the Hubbard eigenfunctions discussed in Refs. [10,11. These spinon states can be therefore identified by performing Lanczos diagonalization on the $\ell$ site Heisenberg model in the symmetry subspace of total momentum $Q$. Now, having determined the one spinon states at $U=\infty$, we adiabatically lower the interaction parameter $U$ (in practice this is quite an easy procedure within Lanczos method) following the "evolution" of the eigenstate of spinon momentum $Q$ from $U=\infty$ down to the desired value of $U$. In this way, starting from an exact eigenstate of the Heisenberg model we first find an eigenstate of the Hubbard model at $U=\infty$ and then a sequence of eigenstates of the $\ell$ site Hubbard model corresponding to smaller and smaller interaction parameters. This procedure has been devised because the spinon momentum $Q$ is a good quantum number only at $U=\infty$ and then we need a method to select the exact one spinon states out of the full set of eigenstates of the Hubbard Hamiltonian. As usual, a simple check on the validity of the single spinon approximation comes from the completeness condition of the intermediate states, analogous to Eq. (8.3), which now reads

$$
\frac{1}{\ell} \sum_{Q} Z(Q)=<\Psi\left|c_{p, \downarrow}^{\dagger} c_{p, \downarrow}\right| \Psi>\equiv n_{\downarrow}(p)
$$

i.e. the momentum distribution of the spin down electrons at half filling. The amount of violation of this sum rule quantifies the weight of all the other states in the Hilbert space which have been neglected in our approximation.

In Figs. 11-12 we show results from Lanczos diagonalization in chains up to 16 sites. The function $Z(Q)$ for two values of the momentum $p$ of the hole and of the magnetization $\mu$ is reported. In all the cases we have considered the completeness condition (8.5) is very well satisfied showing that one spinon states account for more than the $98 \%$ of the full Hilbert space of intermediate states [35]. This allows to reconstruct the full spectral function for the Hubbard model. On the other hand, the available data also show weak size dependence suggesting that finite size effects are not relevant at high energies. A plot of the predicted spectral function is shown as a function of $\omega$ in Fig. 13 for a typical, intermediate coupling $(U=4 t)$ and a two hole momenta $p$.

\section{SUMMARY AND DISCUSSION}

In this work we have analyzed in some detail the dynamical properties of a hole in an antiferromagnet. Due to the mapping between the attractive Hubbard model at arbitrary density and the half filled repulsive Hubbard model 
in a magnetic field, our analysis directly applies also to the more general case of hole propagation in correlated one dimensional models with a gap either in the charge or in the spin spectrum. Most of the known quasi one dimensional materials in fact belong to these classes and then the present study may be helpful in the interpretation of the available photoemission spectra of quasi $1 d$ systems [36]. First we found the exact spectral function of a single hole in the Ising model which is characterized by a gap both in the charge and in the spin channel. As a result, the quasiparticle weight is finite also in $d=1$ and $A(p, \omega)$ has a $\delta$ contribution. However, due to the absence of spin fluctuations the hole dispersion relation is flat and the hole cannot propagate. Then, we focused on the singularities of the hole spectral function which occur because of the presence of other gapless degrees of freedom (spinons). This spinon gas behaves as a Luttinger liquid which gives rise to the typical critical exponents of one dimensional physics that show up in the hole dynamical properties. Among the results we have obtained, we like to stress few general features of $A(p, \omega)$ which characterize hole propagation in one dimension:

- 1) The main singularities in the spectral function occur along lines in the $(p, \omega)$ plane with dispersion relation determined by the form of the holon band, $\omega(p)=\epsilon_{h}\left(p \pm k_{F}\right)$, while the spinon excitation induced by the hole is created at the Fermi points $\pm k_{F}$. This is a consequence of spin charge decoupling which occurs in one dimension and gives rise to divergences in $A(p, \omega)$ also above the bottom of the band. In this case, the divergence may occur on both sides of $\omega(p)$ with different amplitudes. The existence of two branches of singularities in the spectral function (see Fig. 8) can be interpreted as due to the presence of a shadow band [23].

- 2) The singularities are characterized by critical exponents which can be explicitly calculated in integrable models. In the isotropic antiferromagnet the $S U(2)$ symmetry forces the exponent to be exactly $X=1 / 4$ for all microscopic Hamiltonians. Instead, when spin isotropy is broken, or when the system has a spin gap, the critical exponent $X$ in general depends on the parameters of the model and on the momentum of the hole.

- 3) Away from the $S U(2)$ isotropic point, the tunneling density of states has either a divergence or a zero at the bottom of the spectrum according to the value of the critical exponent $X$, i.e. according to the parameters of the model. Remarkably, the density of states shows at most weak logarithmic singularities in the isotropic case.

- 4) At the bottom of the band the critical exponent coincides with the known exponent characterizing the spectral weight in doped systems when the zero doping limit is taken. This provides a demonstration of the continuity of the physical behavior of the degrees of freedom which do not develop a gap in the excitation spectrum as doping vanishes.

All these features should be experimentally detectable in quasi one dimensional materials.

A problem posed by this analysis concerns the relationship between our results and the zero doping limit of the generally accepted Luttinger liquid picture of the Hubbard or $t-J$ model. In fact, the continuity between the single hole and the low doping physics apparently breaks down when the hole momentum does not coincide with the Fermi momentum of the doped model, i.e. when we are above the bottom of the holon band. In this case, standard bosonization methods would predict singularities in the spectral function with momentum independent critical exponent uniquely determined by the physics at the Fermi points [37] while the accurate analysis of the single hole problem reveals the presence of momentum dependent critical exponents when we move away from the Fermi level.

From a methodological point of view, this study of the single hole motion demonstrates a close relationship between the physics of hole motion and the single impurity problem in Luttinger liquids. This mapping is provided, at strong coupling, by the Galileo transformation which allows to eliminate the hole degree of freedom in favor of a non translationally invariant spin system. The recoil of the hole, embodied in the hole kinetic contribution of the effective spin Hamiltonian $\hat{H}_{p}$, cuts off the backward scattering terms in the impurity problem and generates effective boundary conditions which allow the propagation of spinons through the impurity site. This idea can be extended to higher dimensions. The Galileo transformation, in fact, is not restricted to $d=1$ and the single hole problem can be always mapped to a pure spin Hamiltonian. Generalizing what we found here, it is tempting to assume that also in $d>1$ the hole acts as an effective boundary condition placed at the origin of the $d$-dimensional spin lattice. The emerging picture resembles and generalizes that of the dipolar distortion proposed by Shraiman and Siggia [38] based on the semiclassical treatment of a particular choice of boundary condition. More work along these directions may eventually clarify the properties of hole motion in a correlated background in arbitrary spatial dimensions.

\section{ACKNOWLEGMENTS}


It is a pleasure to acknowledge useful discussions with M. Fabrizio, Q. Shaojing, G. Santoro, F. Mila and E. Tosatti. One of us (AP) wishes to thank the staff at SISSA for warm hospitality. This work is partly supported by PRA HTSC of the Istituto Nazionale di Fisica della Materia (SS). 


\section{APPENDIX A: FORMAL CALCULATION OF THE OVERLAP IN A LUTTINGER LIQUID}

In this appendix we give a formal derivation of the overlap

$$
O_{ \pm}\left(\delta_{ \pm}\right)=<d_{ \pm} \mid c_{ \pm}>
$$

between two free particle states

$$
<d_{ \pm}\left|=\prod_{\mp n \geq 0} d_{n}^{\dagger}\right| 0>, \quad<c_{ \pm}\left|=\prod_{\mp n \geq 0} c_{n}^{\dagger}\right| 0>
$$

on a given branch of a Luttinger liquid, here identified by $( \pm)$. In order to simplify the notation we drop the label \pm from the operators $c$ and $d$ whenever it does not lead to ambiguities. The operators $d$ are defined with skew boundary conditions (i.e. with non vanishing phase shifts) while the operators $c$ correspond to periodic boundary conditions:

$$
\begin{aligned}
& c_{n}^{\dagger}=\frac{1}{\sqrt{\ell}} \int_{-\frac{\ell}{2}}^{\frac{\ell}{2}} e^{i \frac{2 \pi}{\ell} n x} \psi_{ \pm}^{\dagger}(x) \\
& d_{n}^{\dagger}=\frac{1}{\sqrt{\ell}} \int_{-\frac{\ell}{2}}^{\frac{\ell}{2}} e^{i \frac{2 \pi}{\ell}(n+\delta) x} \psi_{ \pm}^{\dagger}(x)
\end{aligned}
$$

where $\psi_{ \pm}(x)$ identifies the fermion field in the right $(+)$ or left $(-)$ branch. The relationship between the operators $d$ and $c$ is easily found using canonical anticommutation rules for the fields $\psi_{ \pm}$:

$$
c_{m}^{\dagger}=\sum_{n=-\infty}^{\infty} s_{\delta}(n-m) d_{n}^{\dagger}
$$

where

$$
s_{\delta}(n)=(-1)^{n} \frac{\sin (\pi \delta)}{\pi(n+\delta)}
$$

The overlap $O_{ \pm}(\delta)$ is given by the determinant of the overlap matrix $D_{n, m}(\delta)=s_{\delta}(n-m)$ with the restriction on the allowed indices $n, m \geq 0$ for the left branch and $n, m \leq 0$ for the right one which selects the occupied orbitals. From these definitions we get the symmetry property:

$$
O_{+}(\delta)=O_{-}(-\delta)
$$

which follows from the transformation rule of the matrix $D_{n, m}(\delta)$ under the mapping $(n, m) \rightarrow(-n,-m)$ which changes the left into the right branch. A further property of the determinants $O_{ \pm}(\delta)$ derives from the definition of the matrix $D(\delta): D_{n, m}(\delta)=s_{\delta}(n-m)=s_{-\delta}(m-n)=D_{m, n}(-\delta)$ which gives:

$$
O_{ \pm}(\delta)=O_{ \pm}(-\delta)
$$

as the determinant of a matrix is equal to that of its transpose. In the continuum limit the determinant $O_{ \pm}$is not well defined and a cut-off procedure is required before evaluating the overlap. Some care should be taken in the explicit definition of the cut-off. In fact, by restricting the matrix indices $(n, m)$ to a finite interval we would effectively introduce an unphysical doubling of the Fermi surface. Instead, let us consider a system with both left and right branches with the same finite phase shifts $\delta_{+}=\delta_{-}=\bar{\delta}$, and finite but large number of particles $N$ symmetrically distributed in the positive and negative branch. In this limit it is clear that the left and right branch decouple and the total overlap $O(\delta)$ is given by the product of the two left and right component $O(\bar{\delta}) \sim O_{+}(\bar{\delta}) O_{-}(\bar{\delta})$. The overlap $O(\delta)$ can be exactly computed in a finite lattice with a given (large) number of particles $N$ [11] and is formally given by the previously introduced determinant with matrix indices belonging to the interval $[1, N]$ :

$$
O(\bar{\delta})=\operatorname{det} \frac{\sin (\pi \bar{\delta})}{\pi(n-m+\bar{\delta})}=A_{\bar{\delta}} N^{-\bar{\delta}^{2}}
$$

where $A_{\bar{\delta}}$ is a finite numerical constant. Then, by use of the relations (A.6, A.7) and the previous result we finally get:

$$
\left|O_{+}(\bar{\delta})\right|^{2}=\left|O_{-}(\bar{\delta})\right|^{2}=O(\bar{\delta})=A_{\bar{\delta}} N^{-\bar{\delta}^{2}}
$$

which gives $\left|O_{ \pm}(\delta)\right| \propto \ell^{-\frac{1}{2} \delta^{2}}$ where we expressed the number of particles $N$ as a fraction $\rho$ of the number of sites $\ell$. 


\section{APPENDIX B: THE DYNAMICAL QUASIPARTICLE WEIGHT}

In Eq. (5.52) we showed how the dynamical quasiparticle weight $Z(x, t)$ splits into the product of contributions coming from the right and left fermion branches. Now we have to evaluate the generic matrix element appearing in the formal expression of $Z(x, t)$ for a non interacting Fermi gas characterized by given phase shifts $\delta_{ \pm}$at the two Fermi points:

$$
Z_{ \pm}^{\delta}(x)=<c_{ \pm}\left|e^{i x\left(\hat{P}_{ \pm}-Q_{ \pm}\right)}\right| c_{ \pm}>
$$

where the states $\mid c_{ \pm}>$are defined in appendix A and $\hat{P}_{ \pm}$represents the total momentum operator for the fermions in the right + or left - branch, defined in Eq. (5.51), with fermionic operators $\psi_{+}$and $\psi_{-}$obeying skew boundary conditions (5.4). Here $Q_{+}$is the minimum momentum of the right branch of $d$ electrons (i.e. electrons with a defined value of the momentum $\hat{P}$ ), as excitations in the right $d$ branch can only increase the momentum by $\frac{2 \pi j}{\ell}$, with positive integer $j$. Conversely, $Q_{-}$is the maximum allowed momentum in the left branch of $d$ electrons, as excitations in the left $d$ branch can only decrease the momentum by $\frac{2 \pi j}{\ell}$ with negative integer $j$. Though in a Luttinger liquid $Q_{ \pm}$ are infinite constants, the functions $Z_{+}(x)$ and $Z_{-}(x)$ are finite also in the continuum limit and have an important property, referred in the following as the Fourier positivity (negativity). By inserting a complete set of the mentioned excitations with definite momentum in the RHS of Eq. (B.1) we get

$$
Z_{+}^{\delta}(x)=\sum_{n \geq 0} \sum_{j}\left|<c_{+}\right| d_{j, n}>\left.\right|^{2} e^{i \frac{2 \pi n}{\ell} x}
$$

where $j$ in $\mid d_{j, n}>$ labels all the possible excited states $\mid d_{j, n}>$ with momentum $Q_{+}+\frac{2 \pi n}{\ell}$. Such a spectral decomposition of $Z_{+}^{\delta}$ implies that the Fourier coefficients $Z_{+}^{\delta}(n)=\int_{0}^{\ell} e^{-i \frac{2 \pi n x}{\ell}} Z_{+}^{\delta}(x) d x$ are non vanishing (and positive definite) only for $n \geq 0$ (Fourier positivity). Analogously $Z_{-}^{\delta}(n)>0$ only for $n \leq 0$ (Fourier negativity ).

In terms of the $d$ operators $($ A.3 $)$ the momentum on each branch $\hat{P}_{ \pm}$is diagonal and reads:

$$
\hat{P}_{ \pm}=\sum_{n}\left(k_{n}+k_{F}^{ \pm}\right) d_{n}^{\dagger} d_{n}
$$

where $k_{n}=\frac{2 \pi}{\ell}(n+\delta)$ and $k_{F}^{ \pm}= \pm k_{F}$. Using the same particle hole transformation discussed in appendix A, (i.e. the transformation $n \rightarrow-n$ ) we obtain the analog of Eq. A.6.):

$$
Z_{ \pm}^{\delta}(x)=Z_{\mp}^{-\delta}(-x)
$$

Having discussed the general symmetry properties of $Z_{ \pm}^{\delta}(x)$, we now turn to the explicit evaluation of the function

$$
Z^{\delta}(x)=<\Psi\left|e^{i \hat{P} x}\right| \Psi>
$$

in a free Fermi gas of $N$ particles, where $|\Psi\rangle$ is the ground state with periodic boundary conditions while the momentum operator $\hat{P}$ refers to a system with skew boundary conditions. Following appendix A, we carry out the calculation for a model of fermions with constant phase shift $\delta$ throughout the Brillouin zone and then we relate the result to $Z_{ \pm}^{\delta}(x)$ by use of the symmetry properties previously discussed. In particular, if we keep only the most relevant singularity, the factorization property proved in Eq. (5.52) gives:

$$
Z^{\delta}(x)=e^{i Q x} Z_{+}^{\delta}(x) Z_{-}^{\delta}(x)=e^{i Q x} Z_{+}^{\delta}(x) Z_{+}^{-\delta}(-x)
$$

where use has been made of the symmetry (B.4) and $Q=Q_{+}+Q_{-}$is the reference momentum of the intermediate states.

In a free Fermi gas, both the ground state $\mid \Psi>$ and the intermediate states are Slater determinants built with the different fermionic operators $c_{n}$ and $d_{n}$ respectively (see Eqs. A.2 and A.3. The translation operator exp $(i \hat{P} x)$ with $\hat{P}=\hat{P}_{+}+\hat{P}_{-}(\sqrt{\mathrm{B} .3})$ is a one body unitary operator which maps the Slater determinant with plane waves single particle orbitals

$$
\phi_{n}(r)=\frac{1}{\sqrt{\ell}} e^{i \frac{2 \pi}{\ell} n r}
$$

into another Slater determinant with orbitals 


$$
\psi_{m}(r)=\frac{1}{\sqrt{\ell}} \sum_{j} s_{\delta}(j-m) e^{i k_{j}(x+r)}
$$

where $s_{\delta}(n)$ is defined in (A.5). The overlap between these two Slater determinants is just the determinant of the $N \times N$ matrix $M_{n m}$ of the overlaps between the occupied orbitals:

$$
M_{n m}=z^{\delta} \sum_{j} s_{\delta}(j-m) s_{\delta}(j-n) z^{j}
$$

giving explicitly

$$
M_{n m}= \begin{cases}(-1)^{n-m} e^{i \pi \delta} \frac{\sin \pi \delta}{\pi} \frac{z^{n}-z^{m}}{n-m} & \text { for } n \neq m \\ z^{n}\left[1+i u e^{i \pi \delta} \frac{\sin \pi \delta}{\pi}\right] & \text { for } n=m\end{cases}
$$

where we have introduced the phase factor $z=e^{i u}$ with $u=\frac{2 \pi x}{\ell}$. The matrix $M_{n m}$ can be written as the product three diagonal matrices (with diagonal elements $(-1)^{n}, z^{n}$ and $(-1)^{m}$ ) which contribute to the determinant with a phase factor and the Toeplitz matrix $T_{n-m}$ given by:

$$
\begin{aligned}
T_{n} & =\int_{0}^{2 \pi} \frac{d \theta}{2 \pi} C(\theta) e^{-i \theta n} \\
C(\theta) & =1+\Theta(u-\theta)\left(e^{i 2 \pi \delta}-1\right)
\end{aligned}
$$

where $\Theta$ is the step function. The leading singularity of the determinant of $T$ might be extracted by means of Szegö's theorem [25] which would give:

$$
\operatorname{det} T=\exp \left\{N g_{0}+\sum_{n \geq 1} n g_{-n} g_{n}\right\}
$$

with

$$
g_{n}=\int_{0}^{2 \pi} \frac{d \theta}{2 \pi} e^{-i n \theta} \ln C(\theta)
$$

By performing the Fourier transform, $g_{n}$ is simply evaluated:

$$
\left\{\begin{array}{l}
g_{0}=i \delta u \\
g_{n}=\frac{\delta}{n}\left(1-e^{-i u n}\right) \quad \text { for } n \neq 0
\end{array}\right.
$$

However, this theorem holds only for continuous $C(\theta)$ and does not apply directly to our case. However one can follow the same regularization applied in an analogous calculation for the Ising model [25] by noting that the Hilbert matrix of elements $H_{n-m}$ defined by:

$$
H_{n}=\frac{\sin \pi \delta}{\pi(n+\delta)}
$$

has the same kind of singularity shown by $T$. In fact, the coefficients $\bar{g}_{n}$ which characterize $H$ are just given by $g_{n}=\delta / n$ for $n \neq 0$ and $g_{0}=0$. Therefore, following Ref. [25], we can apply Szegö's theorem to the ratio of determinants

$$
\frac{\operatorname{det} T}{(\operatorname{det} H)^{2}}=\exp \left\{N g_{0}+\sum_{n \geq 1} n\left[g_{-n} g_{n}+\frac{2 \delta^{2}}{n^{2}}\right]\right\}
$$

Finally, the determinant of a Hilbert matrix can be analytically evaluated, as shown in Eq. (A.8), giving, to leading order,

$$
Z^{\delta}(x)=\operatorname{det} T=e^{i Q x} g^{+}(x) g^{+}(-x)
$$


where the overall phase factor depends on the reference momentum of the intermediate states $Q=\sum_{n} k_{n}\left(\right.$ for $\left.k_{n}<k_{F}\right)$ and

$$
\begin{aligned}
g^{+}(x) & =A_{\delta} N^{-\delta^{2}} \exp \left[\delta^{2} \sum_{n \geq 1} \frac{e^{i u n}}{n}\right] \\
& =A_{\delta}\left[N\left(1-e^{i r}\right)\right]^{-\delta^{2}}
\end{aligned}
$$

$A_{\delta}$ is the same numerical constant appearing in Eq. (A.8) and is given explicitly by

$$
\ln A_{\delta}=-(\delta)^{2}(1+C)+\sum_{j=1}^{\infty} j\left[\frac{\delta^{2}}{j^{2}}+\ln \left(1-\frac{\delta^{2}}{j^{2}}\right)\right]
$$

$C=0.5772 \ldots$ being the Euler constant.

The Fourier positivity property of $g^{+}(x)$ can be easily proved by expanding the exponential in Eq. (B.18). By comparing Eq. (B.17) and Eq.(B.6) we find:

$$
g^{+}(x) / Z_{+}^{\delta}(x)=g^{+}(-x) / Z_{+}^{-\delta}(-x)
$$

where the left hand side has the Fourier positivity property (as the ratio of two function satisfying this property also satisfies the Fourier positivity property), while the right hand side has the Fourier negativity property. Therefore both terms of this equation have to be constant in $x$ implying that $Z_{+}^{\delta}(x) \propto g^{+}(x)$. The overall proportionality constant can be determined by noting that the $n=0$ Fourier coefficient in Eq. (B.2) coincides with the square of the overlap $\left|O_{+}(\delta)\right|^{2}$ which has been explicitly calculated in Eq.(A.9). On the other hand, the $n=0$ Fourier component of $g^{+}(x)$ can be read off from Eq. (B.18) yielding $Z_{+}^{\delta}(x)=g^{+}(x)$.

In the thermodynamic limit $\left(\ell \rightarrow \infty\right.$ at fixed $x$ and fixed density of fermions $\left.\rho=\frac{N}{L}\right)$ Eq. (B.19) simplifies:

$$
Z_{+}^{\delta}(x)=A_{\delta}(2 \pi \rho)^{-\delta^{2}}(-i x+\epsilon)^{-\delta^{2}}
$$

with $\epsilon \sim O\left(x^{2} / \ell^{2}\right)$ is a vanishingly small positive term which defines the branch cut for the non integral exponentiation of the complex number $(-i x+\epsilon)$. Taking the Fourier transform, we get (for $0<\delta<1$ ):

$$
Z_{ \pm}^{\delta}(k)=(2 \pi \rho)^{-\delta^{2}} A_{\delta} 2 \sin \left(\pi \delta^{2}\right) \Gamma\left(1-\delta^{2}\right) \Theta( \pm k)( \pm k)^{\delta^{2}-1}
$$

The asymptotic evaluation of the two functions $Z_{ \pm}^{\delta}(x)$ for a Fermi gas with arbitrary phase shift also provides the leading singularity for $Z_{ \pm}^{\left(\delta_{ \pm},{ }^{ \pm} m\right)}(x)=Z_{ \pm}^{\left(\delta_{ \pm}+m\right)}(x)$ entering the spectral function of the interacting model with different phase shifts on the two branches $\delta_{ \pm}$. In particular, for $t=0$, the Fourier transform of $Z(x, 0)$ in Eq. 5.52) splits into the sum of terms with different phase shifts $\bar{\delta}_{+}=\delta_{+}+m$ and $\bar{\delta}_{-}=\delta_{-}+m$ each given by

$$
\begin{array}{ll}
Z(Q+k)=B \sin \left(\pi \bar{\delta}_{+}^{2}\right) k^{\bar{\delta}_{+}^{2}+\bar{\delta}_{-}^{2}-1} & \text { for } \mathrm{k}>0 \\
Z(Q+k)=B \sin \left(\pi \bar{\delta}_{-}^{2}\right)(-k)^{\bar{\delta}_{+}^{2}+\bar{\delta}_{-}^{2}-1} & \text { for } \mathrm{k}<0
\end{array}
$$

if $Q$ is the average spinon momentum. The prefactor can be also calculated: $B=2(2 \pi \rho)^{-\left(\bar{\delta}_{+}^{2}+\bar{\delta}_{-}^{2}\right)} A_{\bar{\delta}_{+}} A_{\bar{\delta}_{-}} \Gamma\left(1-\bar{\delta}_{+}^{2}-\bar{\delta}_{-}^{2}\right)$. In this case, the Fourier transform is nonvanishing both for positive and negative $k$ and shows singularities with the same exponent for $k \rightarrow 0^{ \pm}$. The amplitude ratio tends to a number which, in the free Fermi gas, just depends on the phase shifts:

$$
\frac{A_{+}}{A_{-}}=\lim _{k \rightarrow 0^{+}} \frac{Z(Q+k)}{Z(Q-k)}=\frac{\sin \left(\pi \bar{\delta}_{+}^{2}\right)}{\sin \left(\pi \bar{\delta}_{-}^{2}\right)}
$$

The asymmetry between the two sides of the singularity is therefore enhanced when one of the phase shifts gets small.

\section{APPENDIX C: THE LONG WAVELENGTH EXPANSION}

The first task is to evaluate the summation present in Eq. (6.16) at large $R$. For simplicity we restrict the analysis to the low density regime. It is easy to verify that the most relevant contributions to the sum are those around $k_{j} \sim 0$. For $r \neq s$ this gives the approximate form: 


$$
A_{r s} \sim \frac{4 e^{i J \tau}}{L\left(q_{r}-q_{s}\right)}\left[f\left(q_{r}\right)-f\left(q_{s}\right)\right]
$$

where the function $f(q)$ is given by:

$$
f(q)=\int_{-\pi}^{\pi} \frac{d k}{2 \pi} \frac{e^{i\left(k R-\tau J k^{2} / 2\right)}}{k-q}
$$

Standard asymptotic expansion leads to

$$
f(q)=e^{i\left(q R-\tau J q^{2} / 2\right)} i \int_{-\infty}^{\infty} \frac{d x}{2 \pi} e^{-i \alpha x^{2}} \frac{\sin x}{x}
$$

where $\alpha=\left[z /(1+q z)^{2}\right] /(2 R)$ and $z=-J \tau / R$. In the $R \rightarrow \infty$ limit at $z=$ const, $\alpha \rightarrow 0$ and the problem simplifies. Notice that this last limiting procedure requires that $\alpha$ is regular and therefore that $1+q z$ is always positive. This implies $|z|<1 / k_{F}$ (where $k_{F}$ is the Fermi momentum of the spinon) which is always satisfied at low density. In general this inequality leads to $R>v_{s} \tau$ where $v_{s}=J k_{F}$ is the spinon velocity at low density. In the following we will consider only this regime. In this case, $\alpha$ can be set equal to zero in the integral leading to the final expression:

$$
f(q)=\frac{i}{2} e^{i\left(q R-\tau J q^{2} / 2\right)}
$$

which inserted into (C.1) gives

$$
A_{r s} \sim \frac{e^{i J \tau} i}{\pi(r-s)}\left[e^{i\left(x r+y r^{2}\right)}-e^{i\left(x s+y s^{2}\right)}\right]
$$

where $r$ and $s$ run over the occupied spinless fermion orbitals $[-\nu, \nu]$ and $x=2 \pi R / L, y=-2 \pi^{2} J \tau / L^{2}$. The diagonal elements require a separate analysis which gives

$$
A_{r r} \sim e^{i J \tau} e^{i\left(x r+y r^{2}\right)}\left[1-\frac{x+2 y r}{\pi}\right]
$$

Now it is convenient to express the matrix $A_{r s}$ as the product of a real matrix $B_{r s}$ and other diagonal matrices $D_{r r}=e^{i\left(x r+y r^{2}\right) / 2}$. In fact, $A=D B D$ where

$$
\begin{array}{ll}
B_{r s} & \sim-\frac{2 \sin \left[\frac{1}{2}\left(x(r-s)+y\left(r^{2}-s^{2}\right)\right)\right]}{\pi(r-s)} \quad r \neq s \\
B_{r r} & \sim 1-\frac{x+2 y r}{\pi}
\end{array}
$$

The asymptotic form of the $N$-spinon Green function $Z(R, \tau)$ is then given, besides a global phase factor coming from the determinant of $D$, by the determinant of the real $N \times N$ matrix $B_{r s}$. For convenience, we fix a finite ratio $N / L=\rho$ (even if the expressions previously derived are exact only for $\rho \rightarrow 0$ ) and we perform the numerical computation of $\operatorname{det} B$ for several values of $R$ and $\tau$. The results are reported in Fig. 6

\section{APPENDIX D: DERIVATION OF THE FINITE SIZE CORRECTIONS}

In this Appendix we sketch the derivation of the general formula (7.3) for the finite size corrections in the Bethe ansatz soluble models: Hubbard and $t-J$ at $J=2 t$. We closely follow the procedure detailed by Woynarovich in his work on the finite size corrections of the Hubbard model at finite doping [27]. For sake of clarity, Hubbard and $t-J$ models will be treated separately.

\section{Hubbard Model}

We consider the Hubbard Hamiltonian on a $L$ site chain at fixed chemical potential $\mu$ and magnetic field $h$ (in units of the hopping amplitude $t$ ): 


$$
H=-\sum_{i=1}^{L} \sum_{\sigma}\left(c_{i+1, \sigma}^{\dagger} c_{i, \sigma}+c_{i, \sigma}^{\dagger} c_{i+1, \sigma}\right)+U \sum_{i=1}^{L} n_{i, \uparrow} n_{i, \downarrow}+\mu \sum_{i=1}^{L}\left(n_{i, \uparrow}+n_{i, \downarrow}\right)-\frac{1}{2} h \sum_{i=1}^{L}\left(n_{i, \uparrow}-n_{i, \downarrow}\right)
$$

where $\sigma=\uparrow, \downarrow$ is the electron spin index. The Bethe ansatz equations for the Hubbard chain read:

$$
\begin{aligned}
& L k_{j}=2 \pi I_{j}+\sum_{\beta=1}^{N_{s}} 2 \arctan \left(4 \frac{\sin k_{j}-\lambda_{\beta}}{U}\right) \\
& \sum_{j=1}^{N_{c}} 2 \arctan \left(4 \frac{\lambda_{\alpha}-\sin k_{j}}{U}\right)=2 \pi J_{\alpha}+\sum_{\beta=1}^{N_{s}} 2 \arctan \left(2 \frac{\lambda_{\alpha}-\lambda_{\beta}}{U}\right)
\end{aligned}
$$

where $N_{c}=L-1$ and $N_{s}=N_{\downarrow}$. The quantum numbers $I_{j}$ and $J_{\alpha}$ are integers or half odd integers depending on the parities of $N_{c}$ and $N_{s}: I_{j}=N_{s} / 2 \bmod (1), J_{\alpha}=\left(N_{c}+N_{s}+1\right) / 2 \bmod (1)$. The existence of a solution to these equations requires that each set $I_{j}$ and $J_{\alpha}$ consists of mutually different quantum numbers. Therefore, the distribution $I_{j}$ is uniquely defined by the position of the hole $I_{h}$. Due to the periodicity of the Bethe ansatz equations by the substitution $I_{j} \rightarrow I_{j}+L$ and $k_{j} \rightarrow k_{j}+2 \pi$ we can always assume that the $L-1$ quantum numbers $I_{j}$ fill the range $\left[I_{h}+1, I_{h}+L-1\right]$. The important low energy real solutions are found if the $N_{s}$ quantum numbers $J_{\alpha}$ are chosen as contiguous integers (or half odd integers). We denote as $J_{\min }$ and $J_{\max }$ the minimum and maximum value of the distribution respectively. The ground state corresponds to the most symmetrical distribution around zero compatible with the quantization rules. It is useful to introduce the additional quantities $I^{-}=I_{h}-1 / 2, I^{+}=I_{h}+L-1 / 2$, $J^{-}=J_{\min }-1 / 2$ and $J^{+}=J_{\max }+1 / 2$ which, by definition, satisfy the relations (7.1). Finally, the total energy is expressed in terms of the rapidities $k_{j}$ and $\lambda_{\alpha}$ by

$$
E=-2 \sum_{j}^{N_{c}} \cos k_{j}+\mu N_{c}+h\left(N_{s}-N_{c} / 2\right)
$$

The Bethe ansatz equations (D.2) can be written as $z_{c}\left(k_{j}\right)=I_{j} / L$ and $z_{s}\left(\lambda_{\alpha}\right)=J_{\alpha} / L$ in terms of the functions $z_{c}(k)$ and $z_{s}(\lambda)$ defined by Eqs. (2.6) of Ref. [27]. Following this work we also introduce the boundaries $k^{ \pm}$and $\lambda^{ \pm}$of the rapidity distributions defined by $z_{c}\left(k^{ \pm}\right)=I^{ \pm} / L$ and $z_{s}\left(\lambda^{ \pm}\right)=J^{ \pm} / L$. The distribution function for the rapidity $\rho_{c}(k)$ is defined as the derivative of $z_{c}(k)$ with respect to $k$ and, due to Eq. (7.1), satisfies

$$
\begin{gathered}
\int_{k^{-}}^{k^{+}} \rho_{c}(k)=\frac{N_{c}}{L} \\
-\frac{1}{2}\left(\int_{k^{-}}^{2 \pi} \rho_{c}(k)-\int_{2 \pi}^{k^{+}} \rho_{c}(k)\right)-\frac{1}{2 \pi} \int_{\lambda^{-}}^{\lambda^{+}} 2 \arctan (4 \lambda / U) \rho_{s}(\lambda)=\frac{D_{c}}{L}-1
\end{gathered}
$$

while the analogous distribution function $\rho_{s}(\lambda)$ obeys Eqs. (2.14) of Ref. [27]. Following the derivation of Ref. [27] we find the $O\left(1 / L^{2}\right)$ correction to the rapidity distributions which can be expressed in compact notation as:

$$
\begin{aligned}
\rho(k, \lambda)= & \rho_{\infty}(k, \lambda)+ \\
& \frac{1}{24 L^{2}}\left(\frac{\rho_{1}\left(k, \lambda \mid k^{+} \lambda^{+}\right)}{\rho_{c}\left(k^{+}\right)}-\frac{\rho_{1}\left(k, \lambda \mid k^{-} \lambda^{-}\right)}{\rho_{c}\left(k^{-}\right)}+\frac{\rho_{2}\left(k, \lambda \mid k^{+} \lambda^{+}\right)}{\rho_{s}\left(\lambda^{+}\right)}-\frac{\rho_{2}\left(k, \lambda \mid k^{-} \lambda^{-}\right)}{\rho_{s}\left(\lambda^{-}\right)}\right)
\end{aligned}
$$

where $\rho, \rho_{1}, \rho_{2}$ are vector functions with two components, one referring to charge and the other to spin, and satisfy equations $(2.16),(2.20)$ of Ref. 27]. Notice that in our one hole case the functions $\rho_{1}$ are symmetric under the simultaneous interchange of $k^{+} \rightarrow k^{-}$and $\lambda^{+} \rightarrow \lambda^{-}$while the functions $\rho_{2}$ are antisymmetric. This property leads to the cancellation of the charge contribution to the finite size corrections of the total energy which now reads

$$
E=L \epsilon_{\infty}\left(k^{+}, k^{-}, \lambda^{+}, \lambda^{-}\right)-\frac{1}{12 L} \epsilon_{2}\left(k^{+}, k^{-}, \lambda^{+}, \lambda^{-}\right)
$$

where $\epsilon_{\infty}$ and $\epsilon_{2}$ are defined by Eqs. (2.23)-(2.25) of Ref. [27]. In the thermodynamic limit $k^{-} \rightarrow k_{0}$ (i.e. the holon momentum) and $k^{+} \rightarrow k_{0}+2 \pi$ while, as usual, the spin rapidities $\lambda$ are centered symmetrically around the origin: $\lambda^{+} \rightarrow \lambda_{0}$ and $\lambda^{-} \rightarrow-\lambda^{0}$. By expanding $\epsilon_{\infty}\left(k^{+}, k^{-}, \lambda^{+}, \lambda^{-}\right)$around its limiting value for infinite size we get: 


$$
\begin{aligned}
\epsilon_{\infty}\left(k^{+}, k^{-}, \lambda^{+}, \lambda^{-}\right)= & \epsilon_{\infty}\left(k_{0}+2 \pi, k_{0}, \lambda_{0},-\lambda_{0}\right)+ \\
& \pi v_{c}\left(k_{0}\right) \rho_{\infty c}\left(k_{0}\right)\left[\left(k^{+}-k_{0}-2 \pi\right)^{2}-\left(k^{-}-k_{0}\right)^{2}\right]+ \\
& \pi v_{s}\left(\lambda_{0}\right) \rho_{\infty s}\left(\lambda_{0}\right)\left[\left(\lambda^{+}-\lambda_{0}\right)^{2}+\left(\lambda^{-}+\lambda_{0}\right)^{2}\right]
\end{aligned}
$$

in terms of the charge $\left(v_{c}\left(k_{0}\right)\right)$ and spin $\left(v_{s}\left(\lambda_{0}\right)\right)$ velocities. The next step is to express the difference between $\lambda^{ \pm}\left[k^{ \pm}\right]$ and its asymptotic value $\lambda_{0}\left[k_{0}\right]$ in terms of the known parameters $N_{c(s)}$ and $D_{c(s)}$. To this end we start from the Eqs. (D.4) and evaluate their derivatives with respect to $k^{ \pm}$and $\lambda^{ \pm}$in the thermodynamic limit. The final expressions

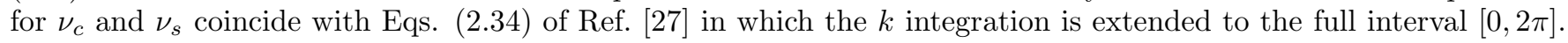
Instead, the equations for $\delta_{c}$ and $\delta_{s}$ now read:

$$
\begin{aligned}
\frac{\partial \delta_{c}}{\partial k^{+}}= & -\frac{\partial \delta_{c}}{\partial k^{-}}+\rho_{\infty c}\left(k_{0}\right) \\
= & -\frac{\rho_{\infty c}\left(k_{0}\right)}{2}\left(-1+\int_{k_{0}}^{2 \pi} \sigma_{1 c}(k)-\int_{0}^{k_{0}} \sigma_{1 c}(k)\right)-\frac{\rho_{\infty c}\left(k_{0}\right)}{\pi} \int_{-\lambda_{0}}^{\lambda_{0}} \arctan (4 \lambda / U) \sigma_{1 s}(\lambda) \\
\equiv & \rho_{\infty c}\left(k_{0}\right) Z_{11} \\
\frac{\partial \delta_{s}}{\partial k^{+}}= & -\frac{\partial \delta_{s}}{\partial k^{-}}=-\frac{\rho_{\infty c}\left(k_{0}\right)}{2}\left(\int_{\lambda_{0}}^{\infty} \sigma_{1 s}(\lambda)-\int_{-\infty}^{-\lambda_{0}} \sigma_{1 s}(\lambda)\right) \equiv \rho_{\infty c}\left(k_{0}\right) Z_{12} \\
\frac{\partial \delta_{c}}{\partial \lambda^{+}}= & -\frac{\rho_{\infty s}\left(\lambda_{0}\right)}{2}\left(\int_{k_{0}}^{2 \pi} \sigma_{2 c}(k)-\int_{0}^{k_{0}} \sigma_{2 c}(k)\right)- \\
& \frac{\rho_{\infty s}\left(\lambda_{0}\right)}{\pi} \arctan \left(4 \lambda_{0} / U\right)-\frac{\rho_{\infty s}\left(\lambda_{0}\right)}{\pi} \int_{-\lambda_{0}}^{\lambda_{0}} \arctan (4 \lambda / U) \sigma_{2 s}(\lambda) \equiv \rho_{\infty s}\left(\lambda_{0}\right) Z_{21}^{+} \\
\frac{\partial \delta_{c}}{\partial \lambda^{-}}= & \frac{\rho_{\infty s}\left(\lambda_{0}\right)}{2}\left(\int_{-2 \pi}^{-k_{0}} \sigma_{2 c}(k)-\int_{-k_{0}}^{0} \sigma_{2 c}(k)\right)- \\
& \frac{\rho_{\infty s}\left(\lambda_{0}\right)}{\pi} \arctan \left(4 \lambda_{0} / U\right)-\frac{\rho_{\infty s}\left(\lambda_{0}\right)}{\pi} \int_{-\lambda_{0}}^{\lambda_{0}} \arctan (4 \lambda / U) \sigma_{2 s}(\lambda) \equiv \rho_{\infty s}\left(\lambda_{0}\right) Z_{21}^{-} \\
\frac{\partial \delta_{s}}{\partial \lambda^{+}}= & \frac{\partial \delta_{s}}{\partial \lambda^{-}}=-\frac{\rho_{\infty s}\left(\lambda_{0}\right)}{2}\left(-1+\int_{\lambda_{0}}^{\infty} \sigma_{2 s}(\lambda)-\int_{-\infty}^{-\lambda_{0}} \sigma_{2 s}(\lambda)\right) \equiv \rho_{\infty s}\left(\lambda_{0}\right) Z_{22}
\end{aligned}
$$

where the additional functions

$$
\begin{aligned}
& \sigma_{1 c(s)}=\frac{\partial \rho_{c(s)}}{\partial k^{+}} \\
& \sigma_{2 c(s)}=\frac{\partial \rho_{c(s)}}{\partial \lambda^{+}}
\end{aligned}
$$

satisfy the equations (2.16), (2.36) of Ref. [27]. From their definitions, it is easy to see that the equations satisfied by the elements of the dressed matrix simplify and give $\xi_{11}=1$ and $\xi_{21}=0$ while

$$
\begin{aligned}
& \xi_{22}(\lambda)=1-\int_{-\lambda_{0}}^{\lambda_{0}} \frac{d \lambda^{\prime}}{2 \pi} K_{2}\left(\lambda-\lambda^{\prime}\right) \xi_{22}\left(\lambda^{\prime}\right) \\
& \xi_{12}(k)=\int_{-\lambda_{0}}^{\lambda_{0}} \frac{d \lambda}{2 \pi} K_{1}(\sin k-\lambda) \xi_{22}(\lambda)
\end{aligned}
$$

where the kernels $K_{1}(x)$ and $K_{2}(x)$ are defined by: $K_{1}(x)=8 U /\left(U^{2}+16 x^{2}\right)$ and $K_{2}(x)=4 U /\left(U^{2}+4 x^{2}\right)$. Some of the elements of the additional matrix $Z_{i j}$ can be related to the dressed charges $\xi_{i j}$ by the algebraic relations:

$$
\begin{aligned}
Z_{11} & =\frac{1}{2}-\xi_{12}\left(k_{0}\right) Z_{12} \\
Z_{22} & =\left[2 \xi_{22}\left(\lambda_{0}\right)\right]^{-1} \\
Z_{21}^{+}+Z_{21}^{-} & =-\xi_{12}\left(k_{0}\right) / \xi_{22}\left(\lambda_{0}\right)
\end{aligned}
$$

while the remaining combinations are expressed in terms of $\sigma_{1 s}$ and $\sigma_{2 s}$ by 


$$
\begin{gathered}
Z_{12}=-\frac{1}{2}\left[\int_{\lambda_{0}}^{\infty} d \lambda \sigma_{1 s}(\lambda)-\int_{-\infty}^{-\lambda_{0}} d \lambda \sigma_{1 s}(\lambda)\right] \\
\Delta Z_{21} \equiv Z_{21}^{+}-Z_{21}^{-}=\int_{-\sin k_{0}}^{\sin k_{0}} d t\left[\frac{1}{2 \pi} K_{1}\left(t-\lambda_{0}\right)+\int_{-\lambda_{0}}^{\lambda_{0}} \frac{d \lambda}{2 \pi} K_{1}(t-\lambda) \sigma_{2 s}(\lambda)\right]
\end{gathered}
$$

The two functions $\sigma_{1 s}(\lambda)$ and $\sigma_{2 s}(\lambda)$ satisfy the following equations:

$$
\begin{aligned}
& \sigma_{1 s}(\lambda)=\frac{1}{2 \pi} K_{1}\left(\lambda-\sin k_{0}\right)-\int_{-\lambda_{0}}^{\lambda_{0}} \frac{d \lambda^{\prime}}{2 \pi} K_{2}\left(\lambda-\lambda^{\prime}\right) \sigma_{1 s}\left(\lambda^{\prime}\right) \\
& \sigma_{2 s}(\lambda)=-\frac{1}{2 \pi} K_{2}\left(\lambda-\lambda_{0}\right)-\int_{-\lambda_{0}}^{\lambda_{0}} \frac{d \lambda^{\prime}}{2 \pi} K_{2}\left(\lambda-\lambda^{\prime}\right) \sigma_{2 s}\left(\lambda^{\prime}\right)
\end{aligned}
$$

By substituting Eq. (D.7) into Eq. (D.6) and evaluating the quantities in brackets by means of Eq. (D.8) we get Eq. (7.3).

In conclusion, the relevant equations for the one hole problem in the Hubbard model are:

$$
\begin{aligned}
& \epsilon_{s}(\lambda)=h_{s}-2 \int_{-\pi}^{\pi} \frac{d k}{2 \pi} K_{1}(\lambda-\sin k) \cos ^{2} k-\int_{-\lambda_{0}}^{\lambda_{0}} \frac{d \lambda^{\prime}}{2 \pi} K_{2}\left(\lambda-\lambda^{\prime}\right) \epsilon_{s}\left(\lambda^{\prime}\right) \\
& \epsilon_{c}(k)=h_{c}-2 \cos k+\int_{-\lambda_{0}}^{\lambda_{0}} \frac{d \lambda}{2 \pi} K_{1}(\sin k-\lambda) \epsilon_{s}(\lambda)
\end{aligned}
$$

which give the spin and charge excitation energies. Obviously, the holon energy is $\epsilon_{h}=-\epsilon_{c}$. The external fields $h_{s}$ and $h_{c}$ are chosen in such a way that $\epsilon\left(\lambda_{0}\right)=0$ and $\epsilon\left(k_{0}\right)=0$. The charge and spin distributions are given by:

$$
\begin{aligned}
& \rho_{s}(\lambda)=\int_{-\pi}^{\pi} \frac{d k}{4 \pi^{2}} K_{1}(\lambda-\sin k)-\int_{-\lambda_{0}}^{\lambda_{0}} \frac{d \lambda^{\prime}}{2 \pi} K_{2}\left(\lambda-\lambda^{\prime}\right) \rho_{s}\left(\lambda^{\prime}\right) \\
& \rho_{c}(k)=\frac{1}{2 \pi}+\cos k \int_{-\lambda_{0}}^{\lambda_{0}} \frac{d \lambda}{2 \pi} K_{1}(\sin k-\lambda) \rho_{s}(\lambda)
\end{aligned}
$$

The spinon and holon velocities are

$$
\begin{aligned}
& 2 \pi v_{s}=\left.\frac{1}{\rho_{s}\left(\lambda_{0}\right)} \frac{d \epsilon_{s}(\lambda)}{d \lambda}\right|_{\lambda_{0}} \\
& 2 \pi v_{c}=-\left.\frac{1}{\rho_{c}\left(k_{0}\right)} \frac{d \epsilon_{c}(k)}{d k}\right|_{k_{0}}
\end{aligned}
$$

The cut-off $\lambda_{0}$ and the holon (dressed) momentum $k_{0}$ are related to the magnetization and to the position of the hole in the charge distribution by

$$
\begin{aligned}
\frac{I_{h}}{L} & =\int_{0}^{k_{0}} d k \rho_{c}(k) \\
\frac{N_{s}}{L} & =\int_{-\lambda_{0}}^{\lambda_{0}} d \lambda \rho_{s}(\lambda)
\end{aligned}
$$

Finally, the elements of the dressed charge matrix (evaluated at the cutoff $k_{0}$ and $\lambda_{0}$ ) are given by Eqs. (D.10) and the additional matrix is defined by Eqs. (D.12) with the the help of Eqs. (D.13). The finite size corrections to the energy and the correlation exponent $X_{s}$ are then given by Eq. (7.3) in terms of the above defined quantities.

\section{Supersymmetric t-J Model}

The expressions for the $t-J$ model are quite similar. In particular the formal size corrections (7.3) are the same while only the definition of the coefficients are different. Also the procedure closely follows that outlined for the Hubbard model. Therefore here we will just report the final expressions in Sutherland's representation [28,26] noting that in this formalism there is only one "charge" (i.e. the hole): $N_{c}=1$. 
The spin and charge excitation energies are given by the equations

$$
\begin{aligned}
& \epsilon_{s}(v)=h_{s}-2 \dot{\theta}(2 v)-\int_{-v_{0}}^{v_{0}} \frac{d v^{\prime}}{2 \pi} \dot{\theta}\left(v-v^{\prime}\right) \epsilon_{s}\left(v^{\prime}\right) \\
& \epsilon_{c}(w)=h_{c}+\int_{-v_{0}}^{v_{0}} \frac{d v}{2 \pi} 2 \dot{\theta}(2 v-2 w) \epsilon_{s}(v)
\end{aligned}
$$

where $h_{s}$ and $h_{c}$ are chosen in such a way $\epsilon\left(v_{0}\right)=0$ and $\epsilon\left(w_{0}\right)=0$. The kernel $\theta(x)=2 \arctan x$ is defined according to Bares et al [26]. Dot represents derivation with respect to the argument. The charge and spin distributions are given by:

$$
\begin{aligned}
& \rho_{s}(v)=\frac{1}{\pi} \dot{\theta}(2 v)-\int_{-v_{0}}^{v_{0}} \frac{d v^{\prime}}{2 \pi} \dot{\theta}\left(v-v^{\prime}\right) \rho_{s}\left(v^{\prime}\right) \\
& \rho_{c}(w)=\int_{-v_{0}}^{v_{0}} \frac{d v}{2 \pi} 2 \dot{\theta}(2 w-2 v) \rho_{s}(v)
\end{aligned}
$$

and the spinon and holon velocities are:

$$
\begin{aligned}
& 2 \pi v_{s}=\left.\frac{1}{\rho_{s}\left(v_{0}\right)} \frac{d \epsilon_{s}(v)}{d v}\right|_{v_{0}} \\
& 2 \pi v_{c}=-\left.\frac{1}{\rho_{c}\left(w_{0}\right)} \frac{d \epsilon_{c}(w)}{d w}\right|_{w_{0}}
\end{aligned}
$$

The cut-off $v_{0}$ and the holon rapidity $w_{0}$ are related to the magnetization and to the position of the hole in the charge distribution by

$$
\begin{aligned}
\frac{I_{h}}{L} & =\int_{-v_{0}}^{v_{0}} d v \rho_{s}(v) \theta\left(2 w_{0}-2 v\right) \\
\frac{1+N_{s}}{L} & =\int_{-v_{0}}^{v_{0}} d v \rho_{s}(v)
\end{aligned}
$$

Other relevant quantities are the dressed charges:

$$
\begin{aligned}
& \xi_{22}(v)=1-\int_{-v_{0}}^{v_{0}} \frac{d v^{\prime}}{2 \pi} \dot{\theta}\left(v-v^{\prime}\right) \xi_{22}\left(v^{\prime}\right) \\
& \xi_{12}(w)=\int_{-v_{0}}^{v_{0}} \frac{d v}{2 \pi} 2 \dot{\theta}(2 w-2 v) \xi_{22}(v)
\end{aligned}
$$

In the following, we will consider the dressed charges evaluated at the cut-off: $\xi_{22} \equiv \xi_{22}\left(v_{0}\right)$ and $\xi_{12} \equiv \xi_{12}\left(w_{0}\right)$. Finally, the elements of the $Z$-matrix are defined by:

$$
\begin{aligned}
Z_{11} & =\frac{1}{2}+\int_{-v_{0}}^{v_{0}} \frac{d v}{2 \pi} \sigma_{1}(v) \theta\left(2 w_{0}-2 v\right) \\
Z_{12} & =\frac{1}{2}\left[\frac{\theta\left(2 v_{0}-2 w_{0}\right)-\theta\left(2 v_{0}+2 w_{0}\right)}{2 \pi}-\int_{-v_{0}}^{v_{0}} \frac{d v}{2 \pi} \sigma_{1}(v)\left[\theta\left(v_{0}-v\right)-\theta\left(v_{0}+v\right)\right]\right] \\
Z_{21}^{+}-Z_{21}^{-} & =0
\end{aligned}
$$

where the function $\sigma_{1}(v)$ satisfies the following equation:

$$
\sigma_{1}(v)=\frac{1}{\pi} \dot{\theta}\left(2 v-2 w_{0}\right)-\int_{-v_{0}}^{v_{0}} \frac{d v^{\prime}}{2 \pi} \dot{\theta}\left(v-v^{\prime}\right) \sigma_{1}\left(v^{\prime}\right)
$$


[1] W.F. Brinkman and T.M. Rice, Phys. Rev. B 2, 1324 (1970).

[2] B.O. Wells et al. Phys. Rev. Lett. 74, 964 (1995); Z.-X. Shen et al. Science 267, 343 (1995).

[3] P. Aebi et al. Phys. Rev. Lett. 72, 2757 (1994).

[4] B. Dardel et al. Phys. Rev. Lett. 67, 3144 (1991).

[5] E. Dagotto, Rev. Mod. Phys. 66, 763 (1994).

[6] E.M. Lieb and F.Y. Wu, Phys. Rev. Lett. 20, 1455 (1968).

[7] R.T. Scalettar, N.E. Bickers and D.J. Scalapino Phys. Rev. B 40, 197 (1989); A. Moreo and D.J. Scalapino Phys. Rev. Lett. 66, 946 (1991).

[8] See for instance I. Affleck, in Field Strings and Critical Phenomena, E. Brézin and J. Zinn-Justin Eds. (North-Holland, Amsterdam, 1990) p.563.

[9] Y. Nagaoka, Phys. Rev. 147, 392 (1966)

[10] M. Ogata and H. Shiba Phys. Rev. B 41, 2326 (1990).

[11] S. Sorella and A. Parola, J. Phys. Cond. Mat. 43589 (1992).

[12] S. Sorella and A. Parola Phys. Rev. Lett. 76, 4604 (1996).

[13] J. Solyom Adv. Phys. 28, 201 (1979).

[14] F.D. Haldane J. Phys. C 14, 2585 (1981).

[15] H. Schulz Phys. Rev. Lett. 64, 2831 (1990).

[16] H. Frahm and V.E. Korepin, Phys. Rev. B 42, 10533 (1990) and Phys. Rev. B 43, 5653 (1991).

[17] W. Metzner, P. Schmit and D. Vollhardt Phys. Rev. B 45, 2237 (1992); R. Strack and D. Vollhardt Phys. Rev. B 46, 13852 (1992); D. Vollhardt in "Correlated Electron Systems" ed. V. J. Emery (World Scientific Singapore, 1993) and reference therein.

[18] Q. F. Zhong and S. Sorella Phys. Rev. B 5116135 (1995).

[19] S. Eggert and I. Affleck Phys. Rev. B 46, 10866 (1992).

[20] C.L. Kane and M.P.A. Fisher Phys. Rev. B 46, 15233 (1992).

[21] P.W. Anderson, Phys. Rev. Lett. 18, 1049 (1967); Phys. Rev. 164, 352 (1967).

[22] D. Mattis and E. Lieb J. Math. Phys. 6, 304 (1963).

[23] K. Penc, F. Mila and H. Shiba Phys. Rev. Lett. 75 ,894 (1995); K. Penc et al Phys. Rev. Lett. 771390 (1996); J. Favand et al. Phys. Rev. B 55, R4859 (1997).

[24] K. Penc at al. Phys. Rev. B 55, 15475 (1997).

[25] B. M. McCoy and T. T. Wu "The two dimensional Ising model" , Harward University Press, Cambridge , Massachusetts (1973).

[26] P.A. Bares and G. Blatter Phys. Rev. Lett. 64, 2567 (1990); P.A. Bares, G. Blatter and M. Ogata Phys. Rev. B 44, 130 (1991).

[27] F. Woynarovich J. Phys. A 22, 4243 (1989).

[28] B. Sutherland Phys. Rev. B 12, 3795 (1975).

[29] Z.N.C. Ha Phys. Rev. Lett. 73, 1574 (1994); Z.N.C. Ha and F.D.M. Haldane Phys. Rev. Lett. 73, 2887 (1994).

[30] F. Calogero J. Math Phys. 10, 2191 (1969); B. Sutherland Phys. Rev. A 4, 2019 (1971).

[31] K.J. von Szczepanski et al Phys. Rev. B 41, 2017 (1990)

[32] S. Haas and E. Dagotto Phys. Rev. B 52, R14396 (1995).

[33] R. Preuss et al Phys. Rev. Lett. 73732 (1994).

[34] Q. Shaojing, to be published.

[35] An analogously surprising performance of the single spinon approximation has been already noticed in the calculation of the Green function for the Calogero Sutherland model [29] where the one particle intermediate states provide an excellent approximation to the exact result.

[36] See e.g. C. Kim el al. Phys. Rev. Lett. 77, 4054 (1996).

[37] J. Voit J. Phys.: Condens. Matt. 8, L-779 (1996).

[38] B.I. Shraiman, E.D. Siggia Phys. Rev. Lett. 61, 467 (1988); Phys. Rev. B 42, 2485 (1990). 


\section{FIGURE CAPTIONS}

- Fig. 1 Exact spectral function $A(\omega)$ (in units of the hopping $t$ ) for the single hole in the Ising model for two values of $J$. The heavy line represents the $\delta$ contribution and its height is proportional to the quasiparticle weight.

- Fig. 2 Overlap square $Z$ between the Heisenberg ground state on a $\ell=L-1$ site ring and the ground state of the effective spin Hamiltonian (3.7) corresponding to a single hole in the $L$ site $t-J$ model at $J=4 t$ and $p=\pi / 2$. Lanczos diagonalization has been performed on even chains with $L \leq 26$. The dashed line is a guide to the eye.

- Fig. 3 Overlap square $Z$ between the Heisenberg ground state on a $L$ site ring and the ground state of the effective spin Hamiltonian (3.7) of a single hole in the $L$ site $t-J$ model at $t=0$ (static limit). Lanczos diagonalization has been performed on even chains with $L \leq 26$. The dashed line is a parabolic fit of Lanczos data. The full line shows the expected asymptotic slope of the curve on the basis of conformal field theory $\left(2 X_{0}=3 / 8\right)$.

- Fig. 4 Leading exponent $X_{0}$ of the size dependence of the overlap $\zeta$ in the $t-J_{X Y}$ model as a function of the number $N$ of up spins. Lines represent the numerical evaluation of the determinant of the matrix (6.11). Dots are the analytical prediction based on Eq. (6.7).

- Fig. 5 (a) Spinon term $Z(Q)$ for the $t-J_{X Y}$ model computed as the determinant of the matrix (6.16) at $\tau=0$ for magnetization $\mu=0$ (squares) and $\mu=1 / 4$ (triangles). Two cases corresponding to about 400 and 800 particles are shown by open and full symbols respectively. (b) Logarithmic plot of $Z(Q)$ near the singularity. The lines indicate the slope predicted on the basis of the finite size corrections to the energy (6.7).

- Fig. 6 Asymptotic behavior of the dynamical quasiparticle weight $Z(R, \tau)$ in the $t-J_{X Y}$ model at $\mu=-1 / 4$ calculated as the determinant of the matrix (C.7) in a system of 201 particles. $Z_{0}(R, \tau)$ is defined in Eq. (6.18). Data refer to more than a thousand different points in the $(R, \tau)$ plane.

- Fig. 7 Critical exponents $X_{0}$ (full squares) and $X_{-1}$ (open squares) as a function of the momentum of the hole $p$ for several magnetizations $\mu=-0.4,-0.3,-0.2,-0.1,0.1,0.2,0.3,0.4$ from (a) to (h) respectively, in the Hubbard model at $U=4 t$.

- Fig. 8 Loci of the singularities of the spectral function in the $(p, \omega)$ plane (5.56) for the Hubbard model at $U=4 t$. Energies are measured from the bottom of the holon band. Two magnetizations are shown: $\mu=0$ and $\mu=1 / 4$. Full (dashed) lines correspond to critical exponents $X$ smaller (larger) than $1 / 2$. In the latter case, according to Eq. (5.57), the divergence disappears.

- Fig. 9 Leading exponent $X_{m}$ of the finite size scaling of the overlap $\zeta$ defined in Eq. (5.36) as a function of the hole momentum $p$. Data refer to the $t-J$ model at $\mu= \pm 1 / 4$ and $J=2 t$ in panel (a) and $J=t$ in panel (b). Solid lines: analytical results obtained from the finite size corrections to the energy in the Bethe ansatz solution. Full (open) dots: Power law fit of Lanczos data for $\mu=1 / 4(\mu=-1 / 4)$. Numerical data (full squares) obtained with the same fit for the non integrable point $J=t$, are shown for comparison only in the $\mu=1 / 4$ case. The dashed line connecting these points is a guide to the eye. Diagonalizations have been performed on rings with $L=8,16,24,32$.

- Fig. 10 Breakdown of the completeness condition (8.3) in the single spinon approximation for the $t-J_{X Y}$ model at two magnetizations. $L$ is the length of the chain.

- Fig. 11 Spinon function $Z(Q)$ for the Hubbard model at $\mu=0$ according to the single spinon approximation. (a) refers to $U=4 t$ and $p=p_{F}$; (b) to $U=8 t$ and $p=p_{F}$; (c) to $U=4 t$ and hole momentum $p=0$; (d) to $U=8 t$ and $p=0$. The hole Fermi momentum $p_{F}=\pi / 2$ corresponds to the bottom of the (down) hole band. $Z(Q)$ vanishes inside the spin up Fermi sphere. Symbols represent Lanczos data for different system sizes: $L=6$ (open circles), $L=10$ (open squares), $L=14$ (full circles).

- Fig. 12 Same as Fig. 11 at magnetization $\mu=1 / 4$ corresponding to $p_{F}=\pi / 4$. Symbols represent Lanczos data for different system sizes: $L=8$ (open circles), $L=12$ (open squares), $L=16$ (full circles). 
- Fig. 13 Hole spectral function in the Hubbard model at $U=4 t$ as a function of frequency measured from the bottom of the holon band. Energy is in units of the hopping $t$ and the calculation has been performed in single spinon approximation. Panel (a) corresponds to $p=0$ and $\mu=0$, (b) to $p=0$ and $\mu=1 / 4$, (c) to $p=p_{F}$ and $\mu=0$, (d) to $p=p_{F}$ and $\mu=1 / 4$. Vertical lines show the location of singularities. Dashed lines identify the divergences induced by band structure effects; dotted lines show the frequencies of the non trivial singularities reported in Fig. 8. 


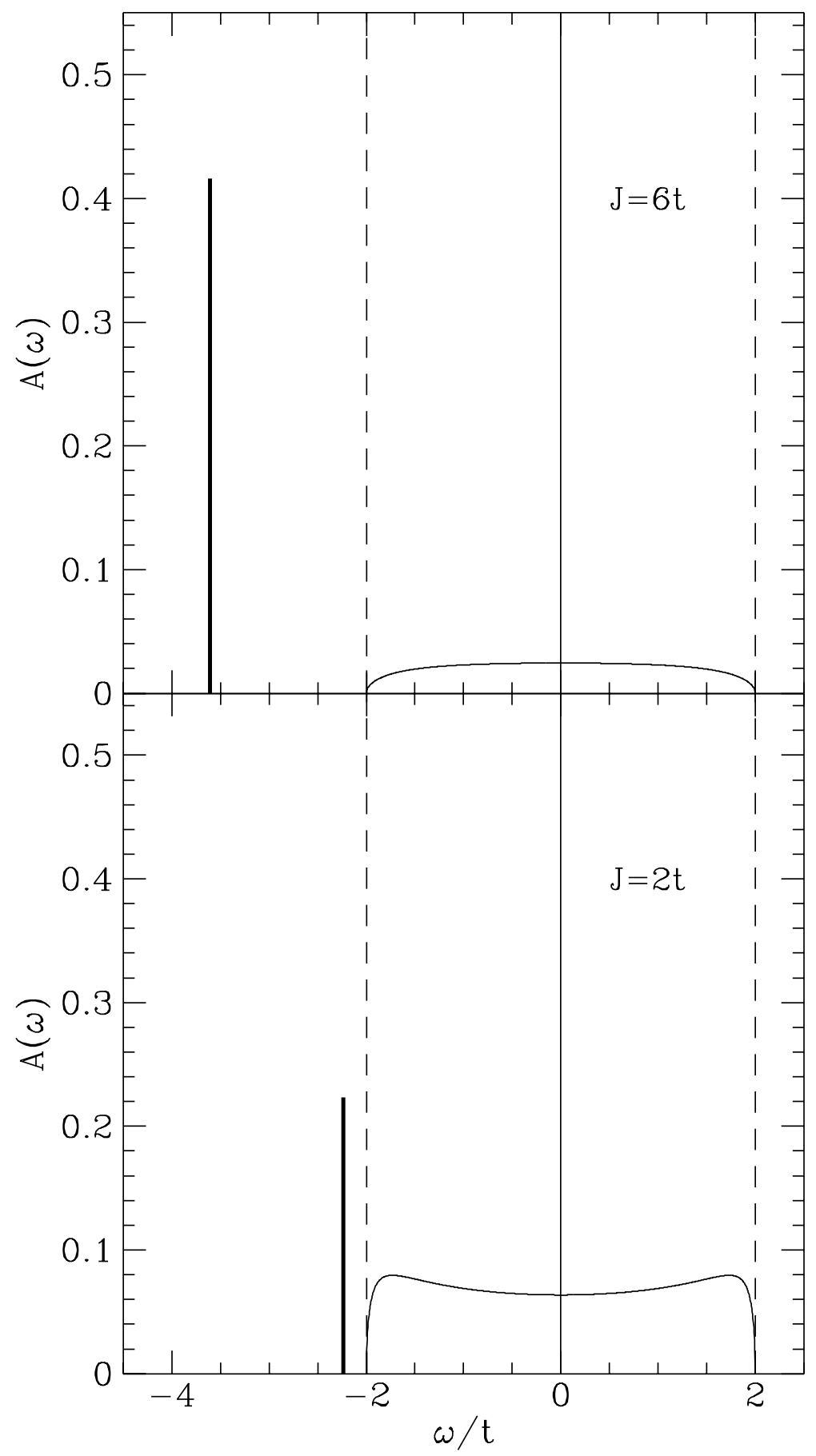

Sorella and Parola Fig. 1 


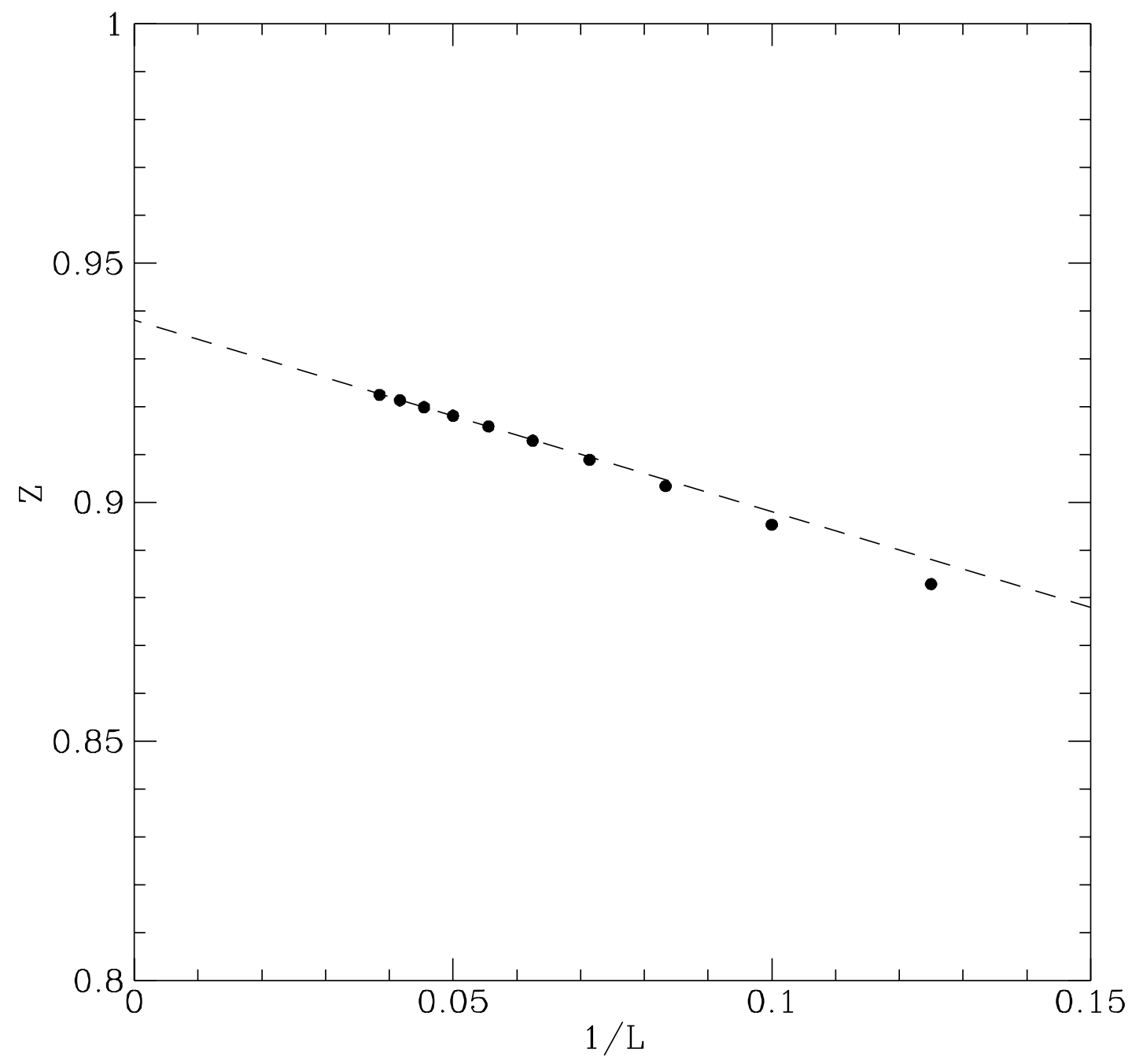

Sorella and Parola Fig. 2 


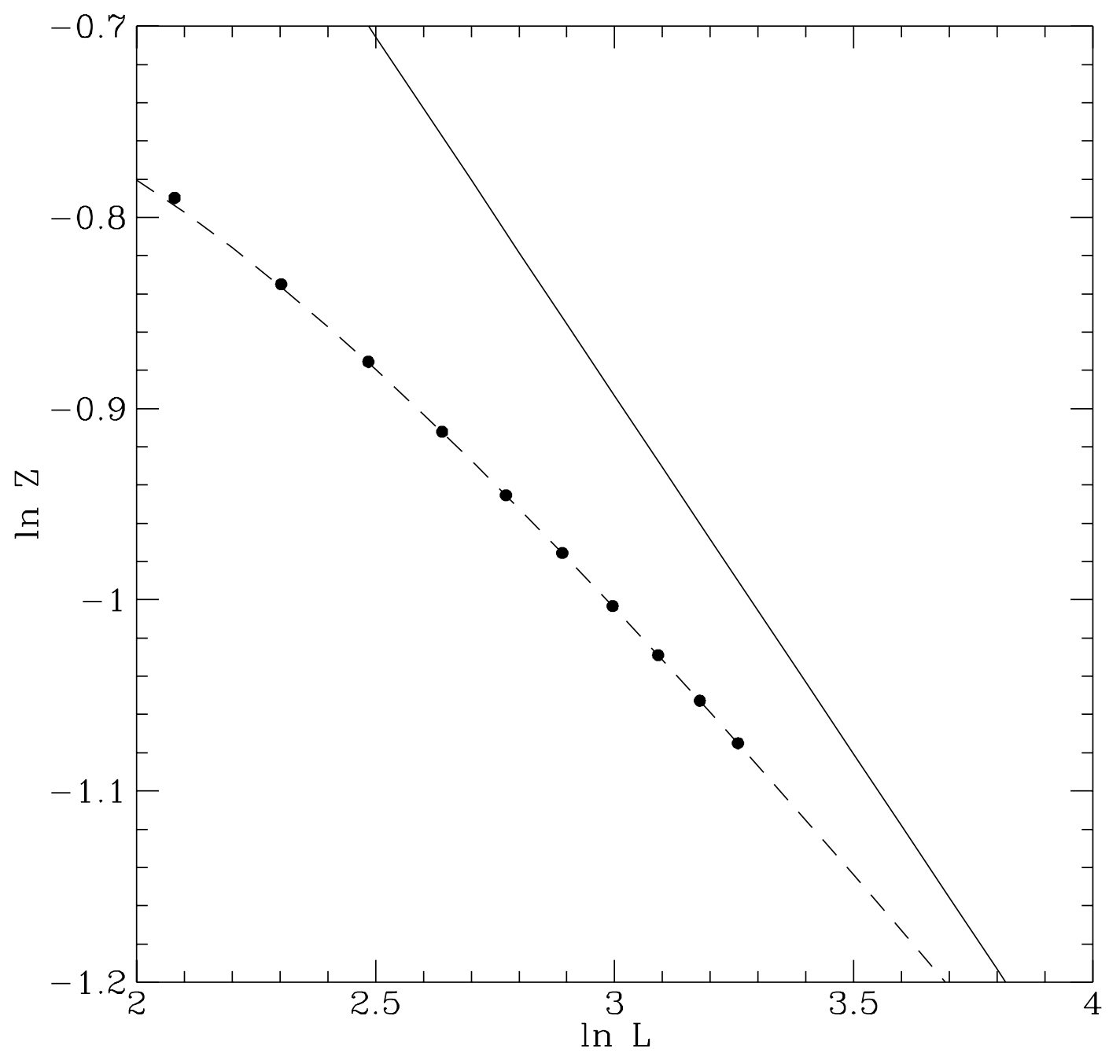

Sorella and Parola Fig. 3 


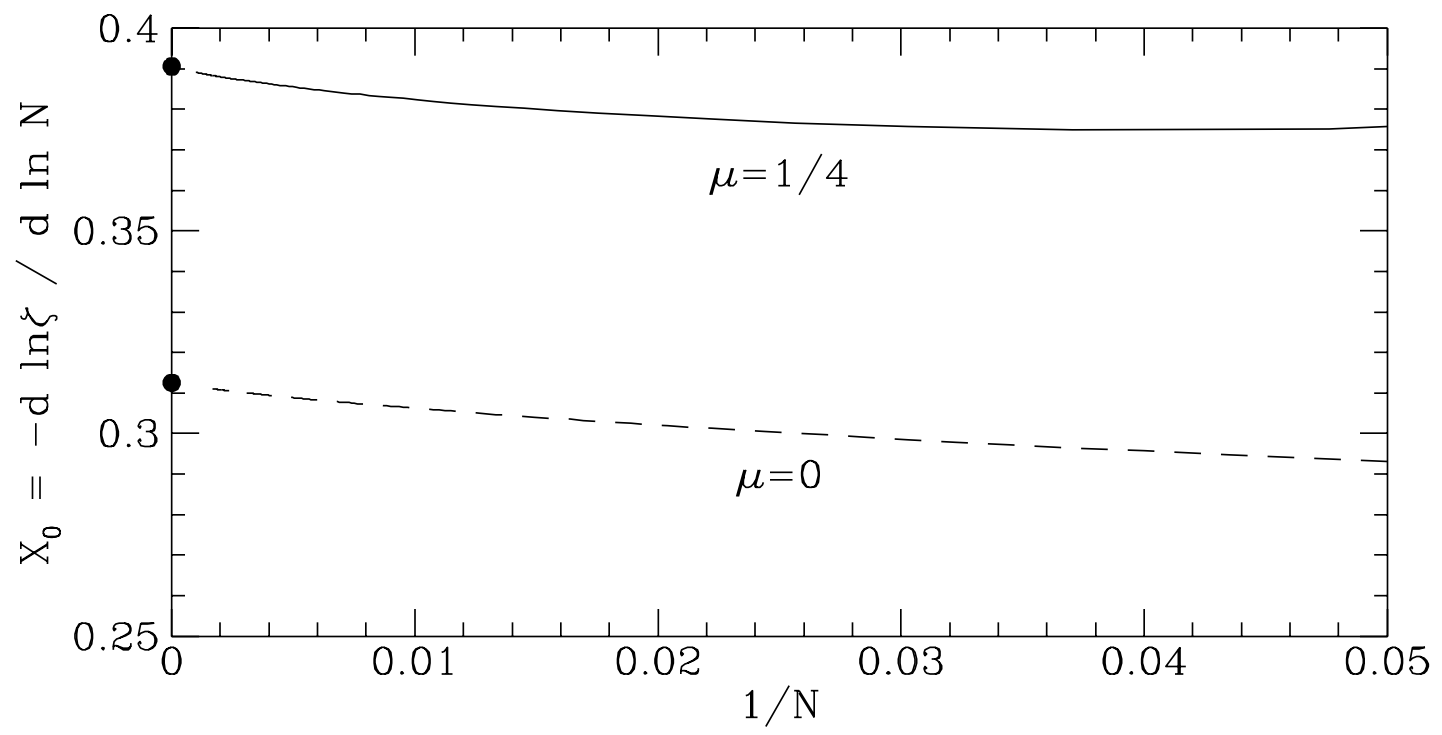

Sorella and Parola Fig. 4 


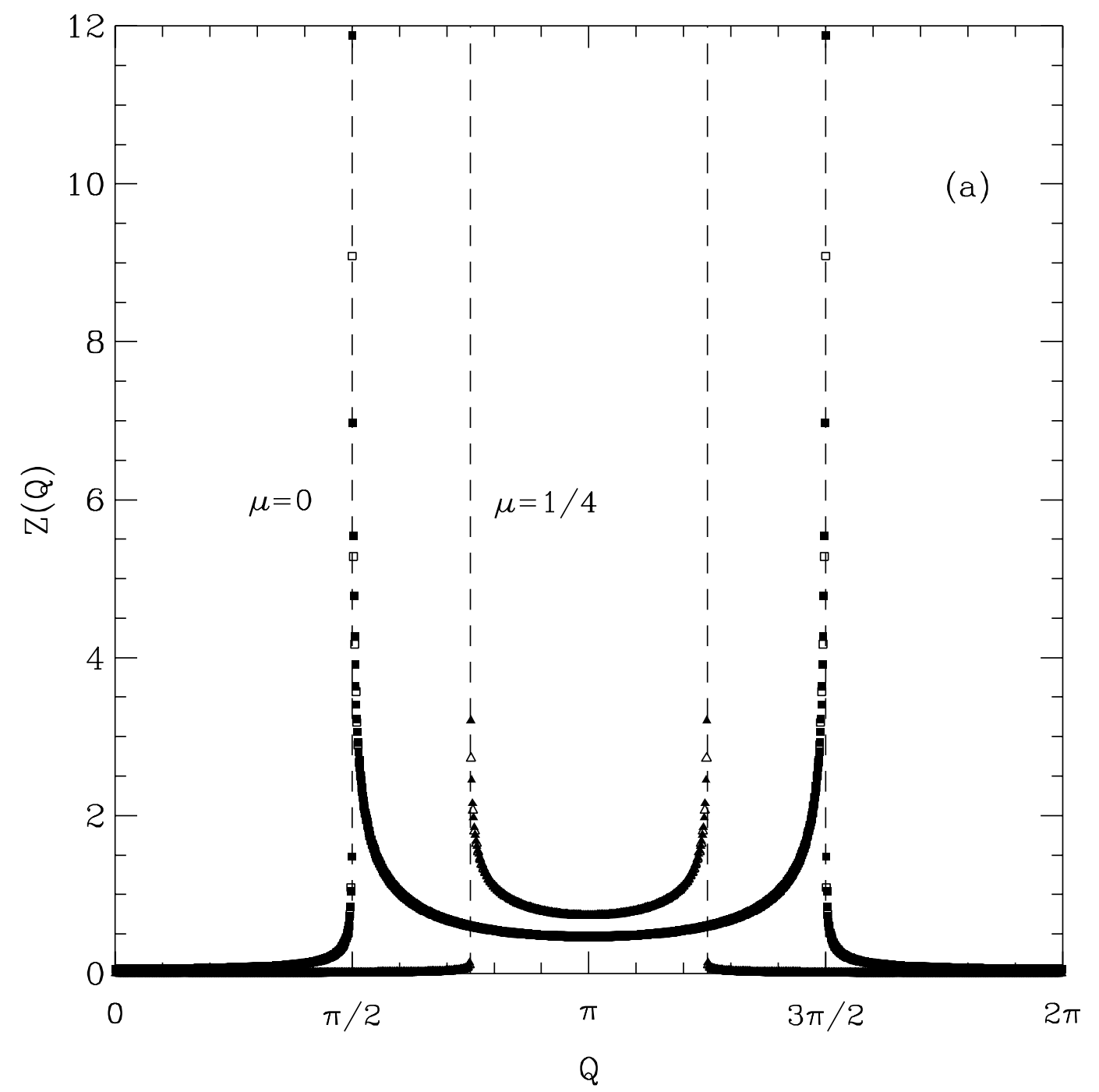

Sorella and Parola Fig. 5a 


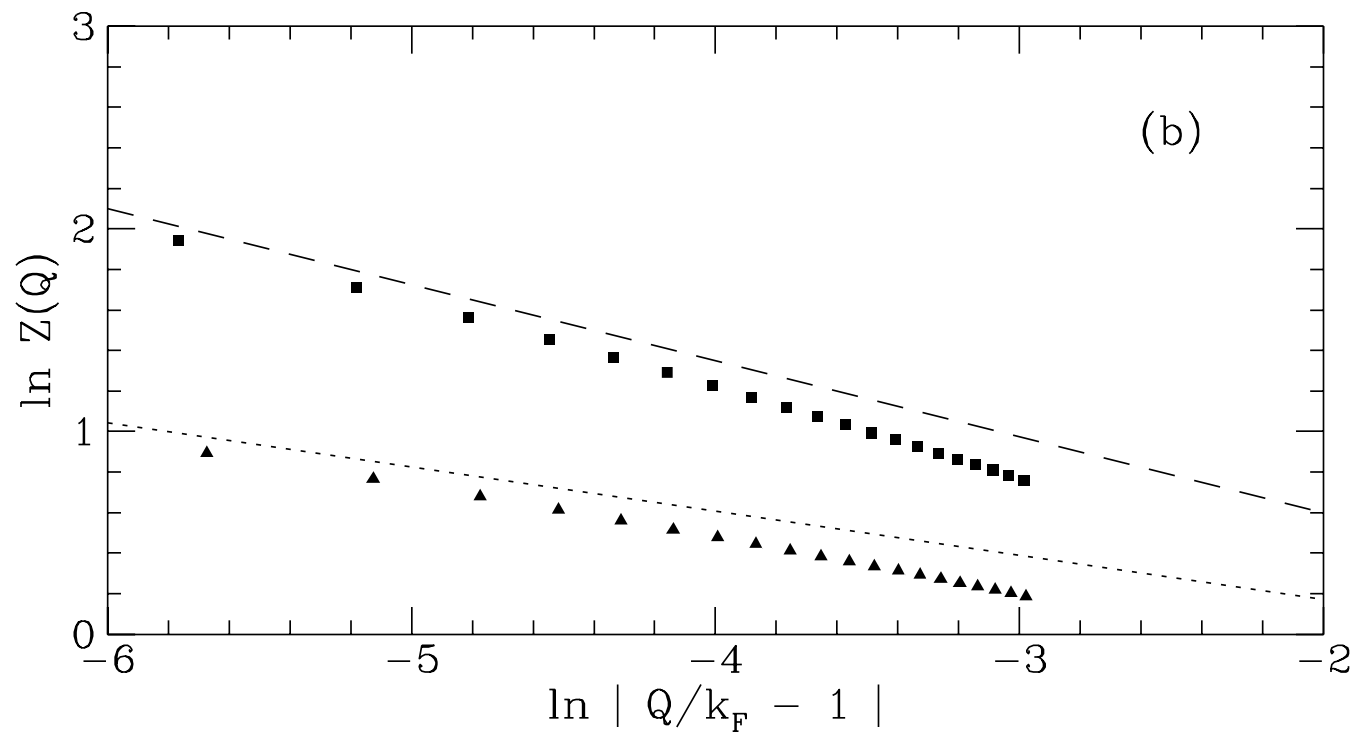

Sorella and Parola Fig. 5b 


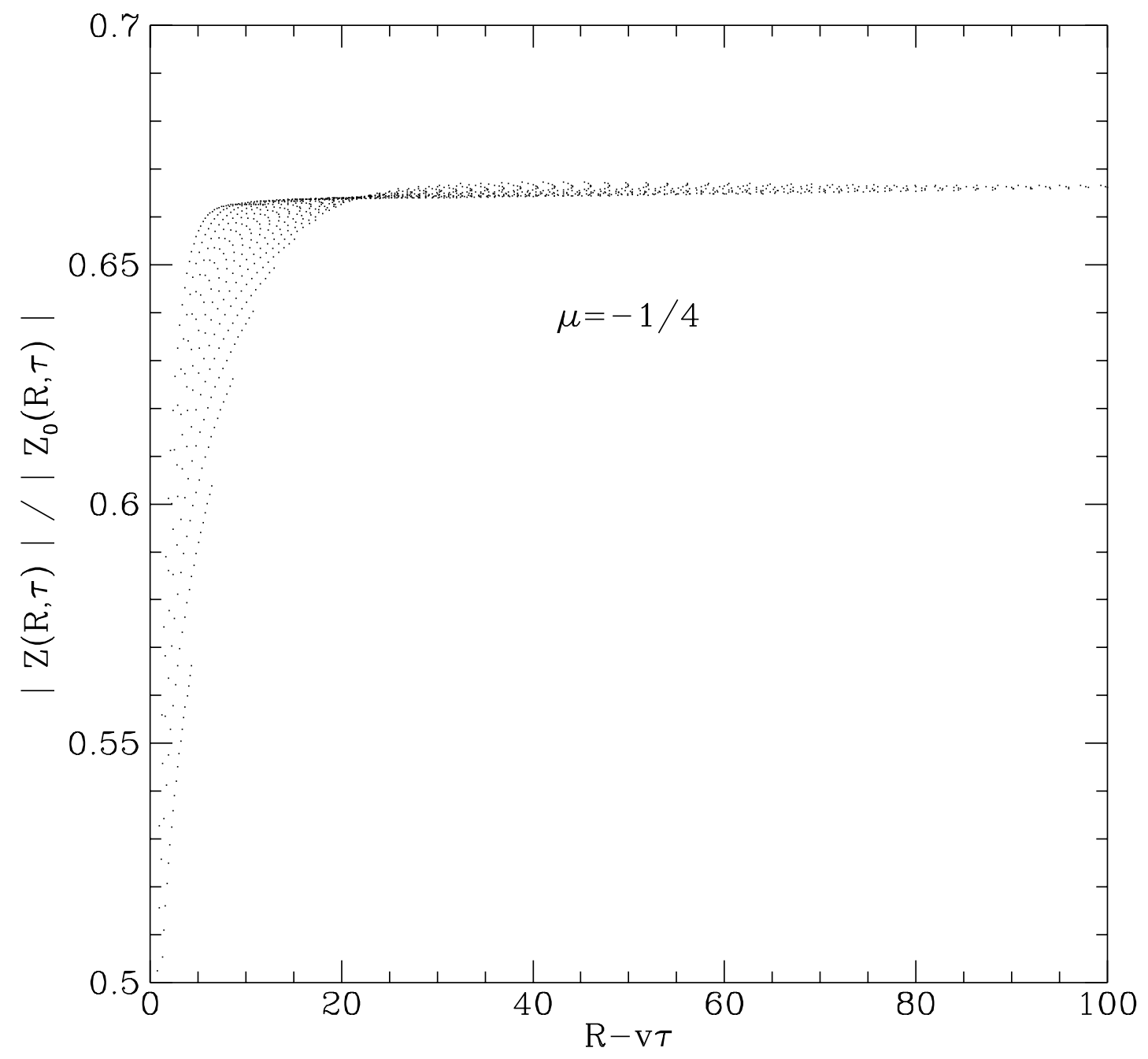

Sorella and Parola Fig. 6 


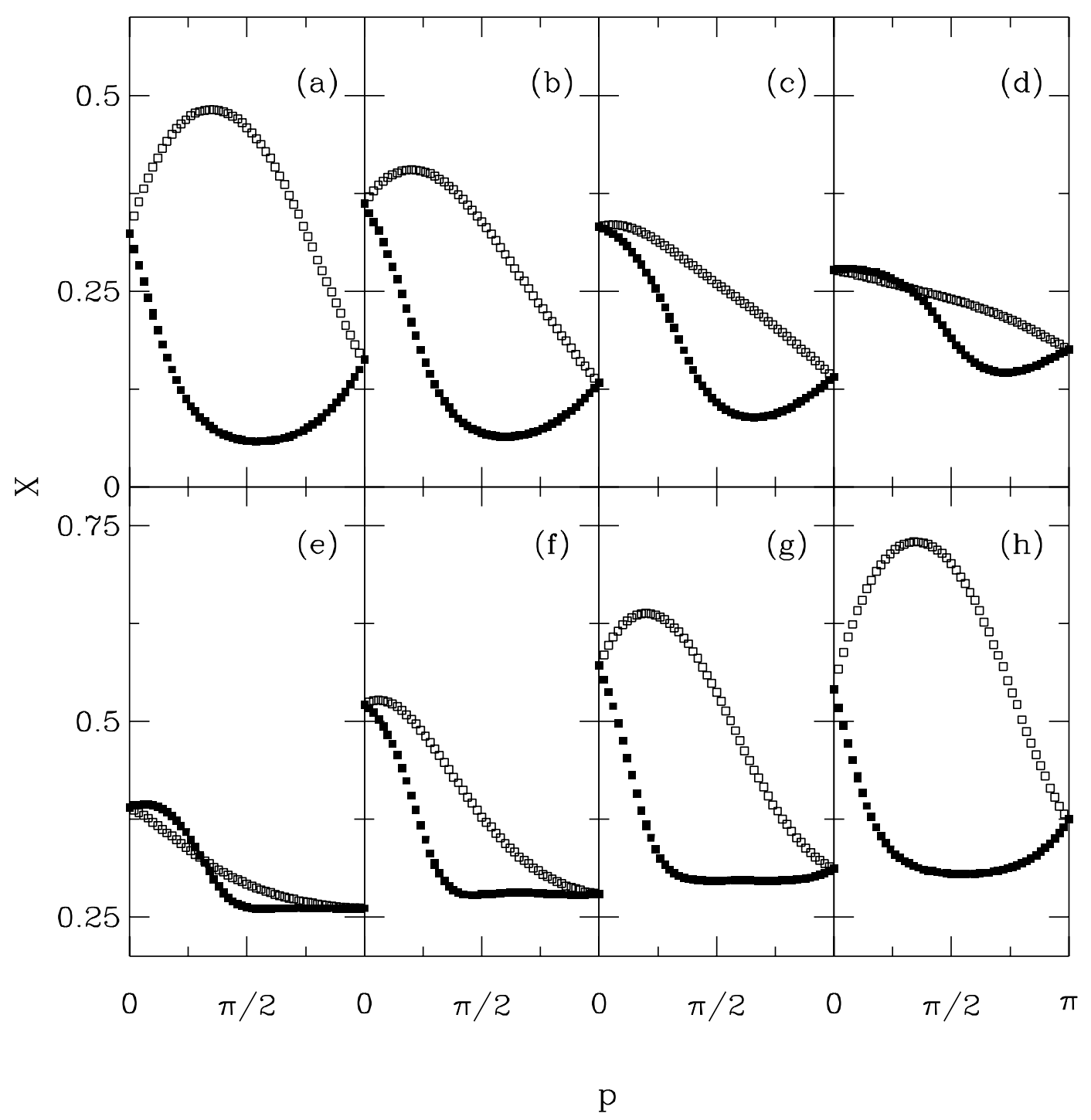

Sorella and Parola Fig. ? 

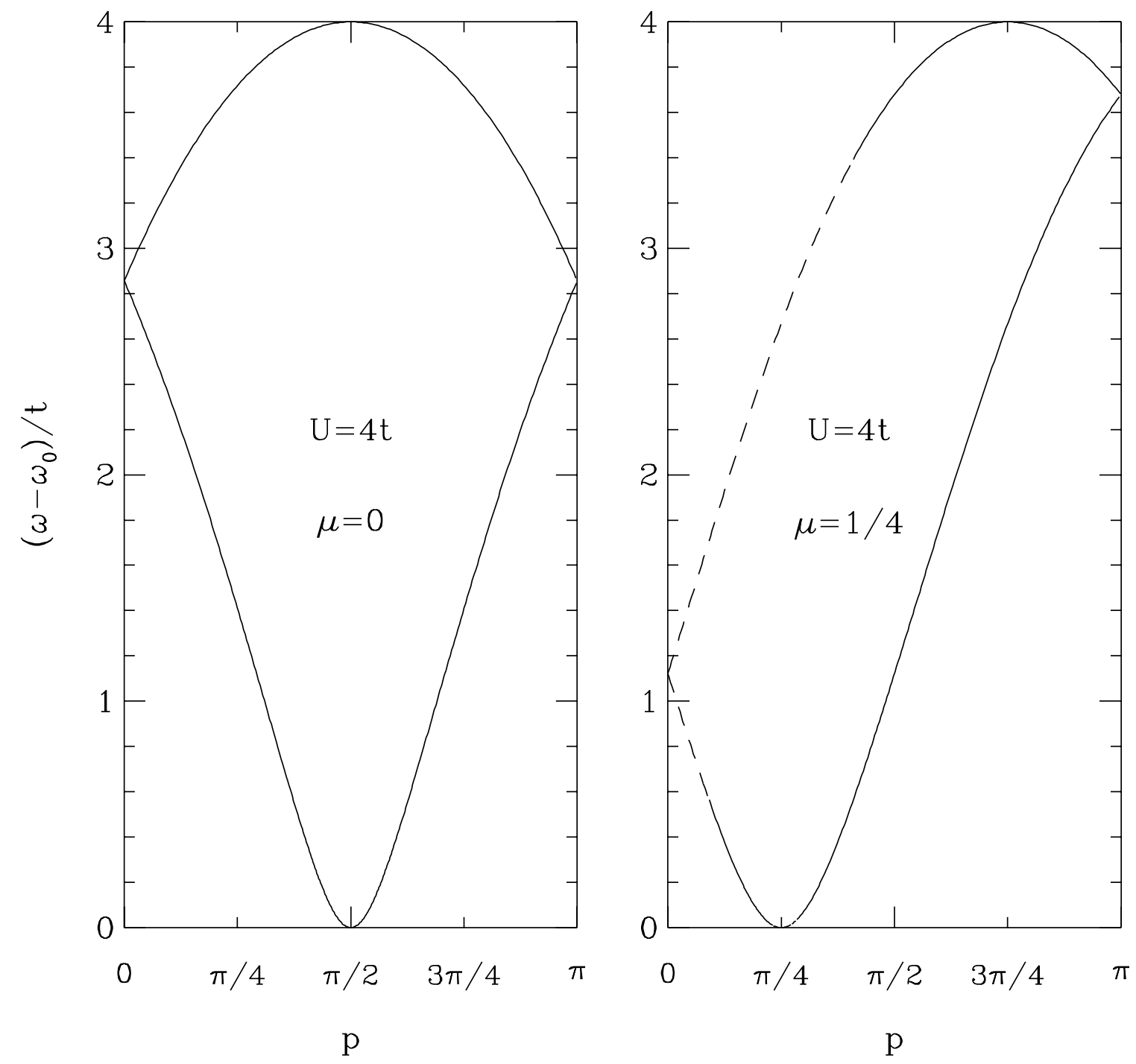

Sorella and Parola Fig. 8 


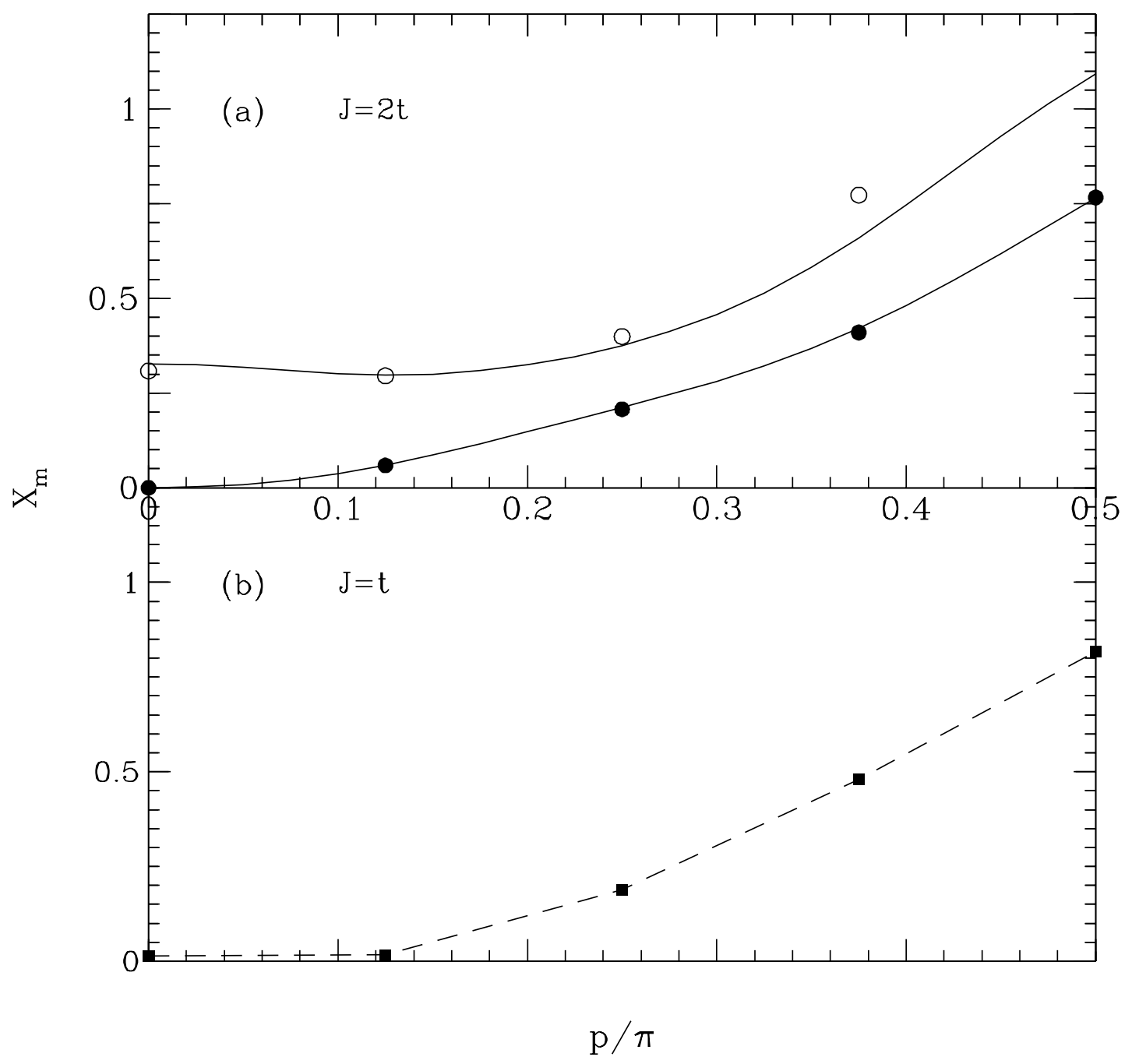

Sorella and Parola Fig. 9 


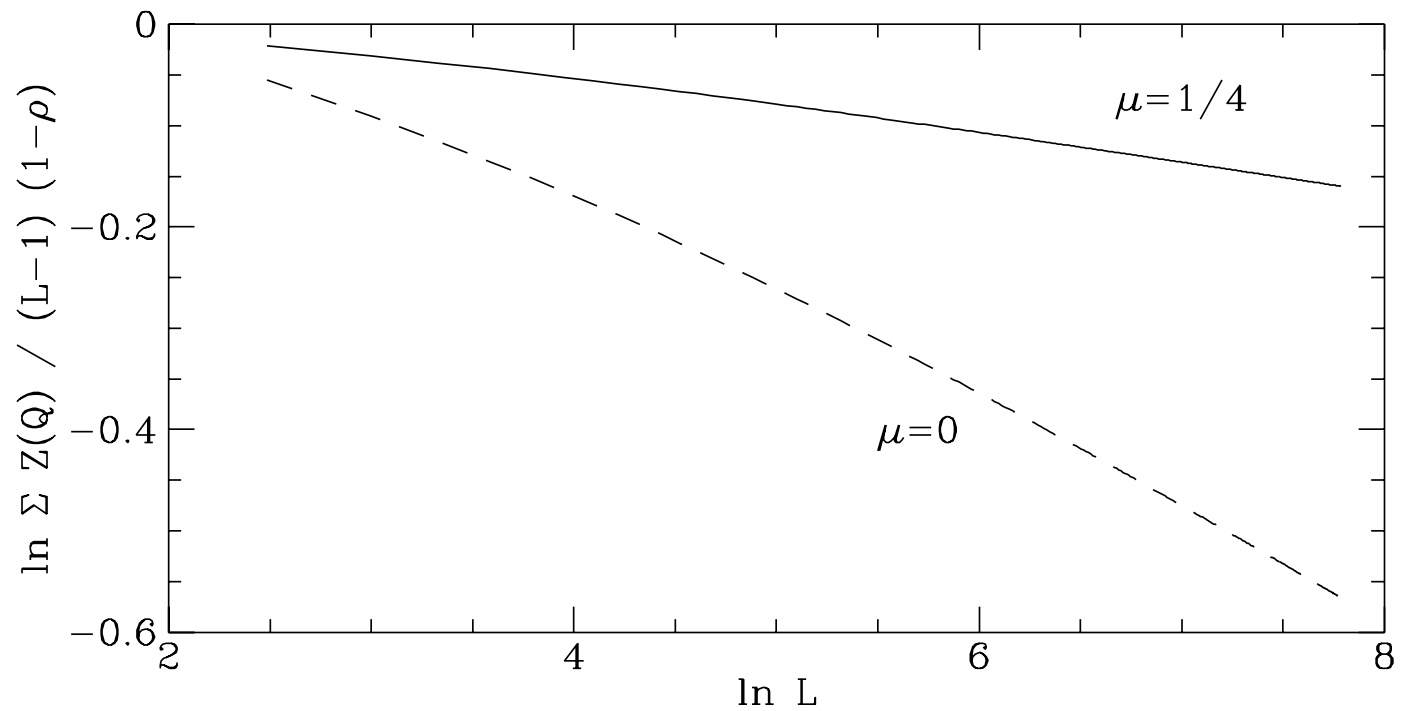

Sorella and Parola Fig. 10 


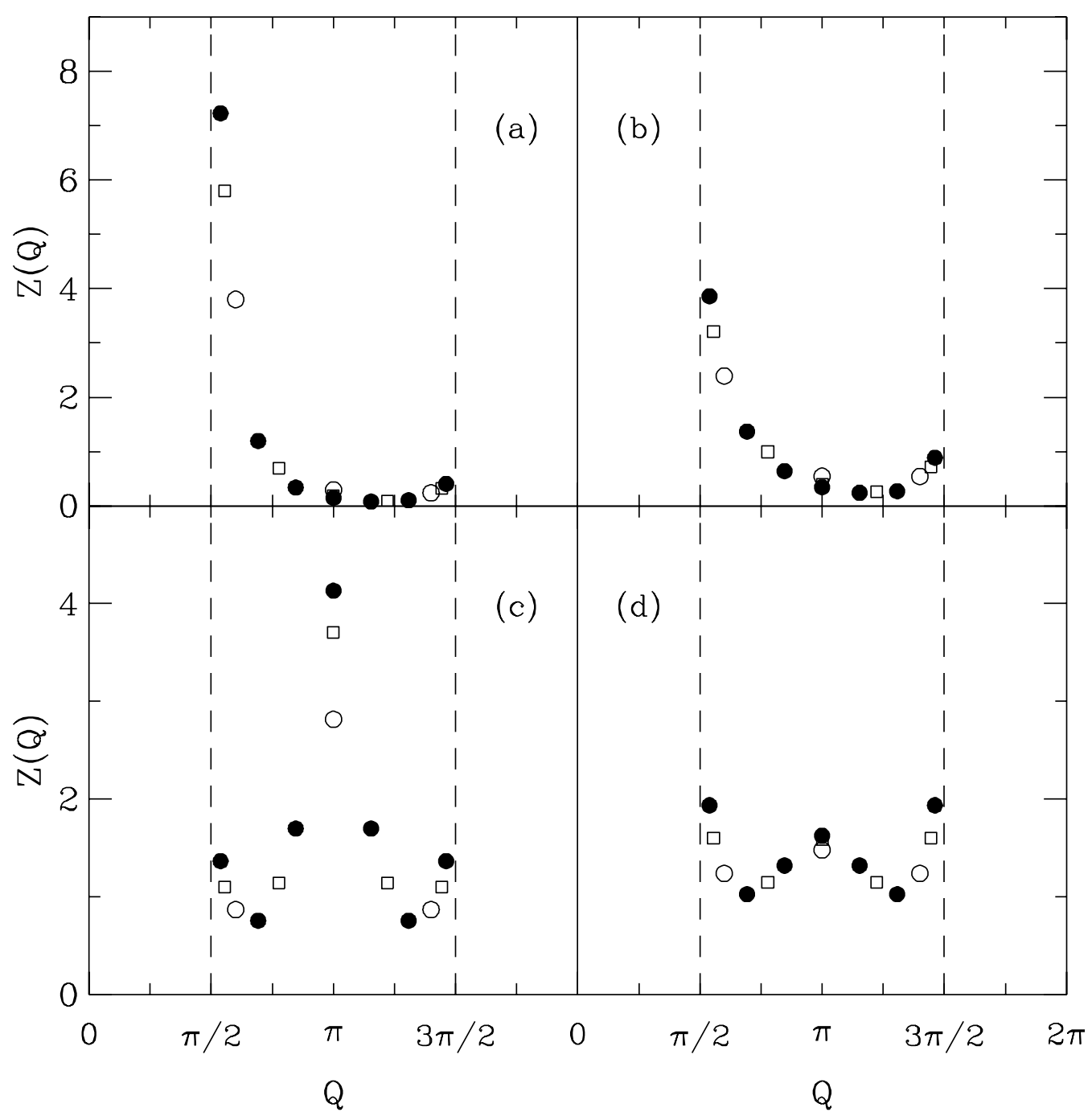

Sorella and Parola Fig. 11 


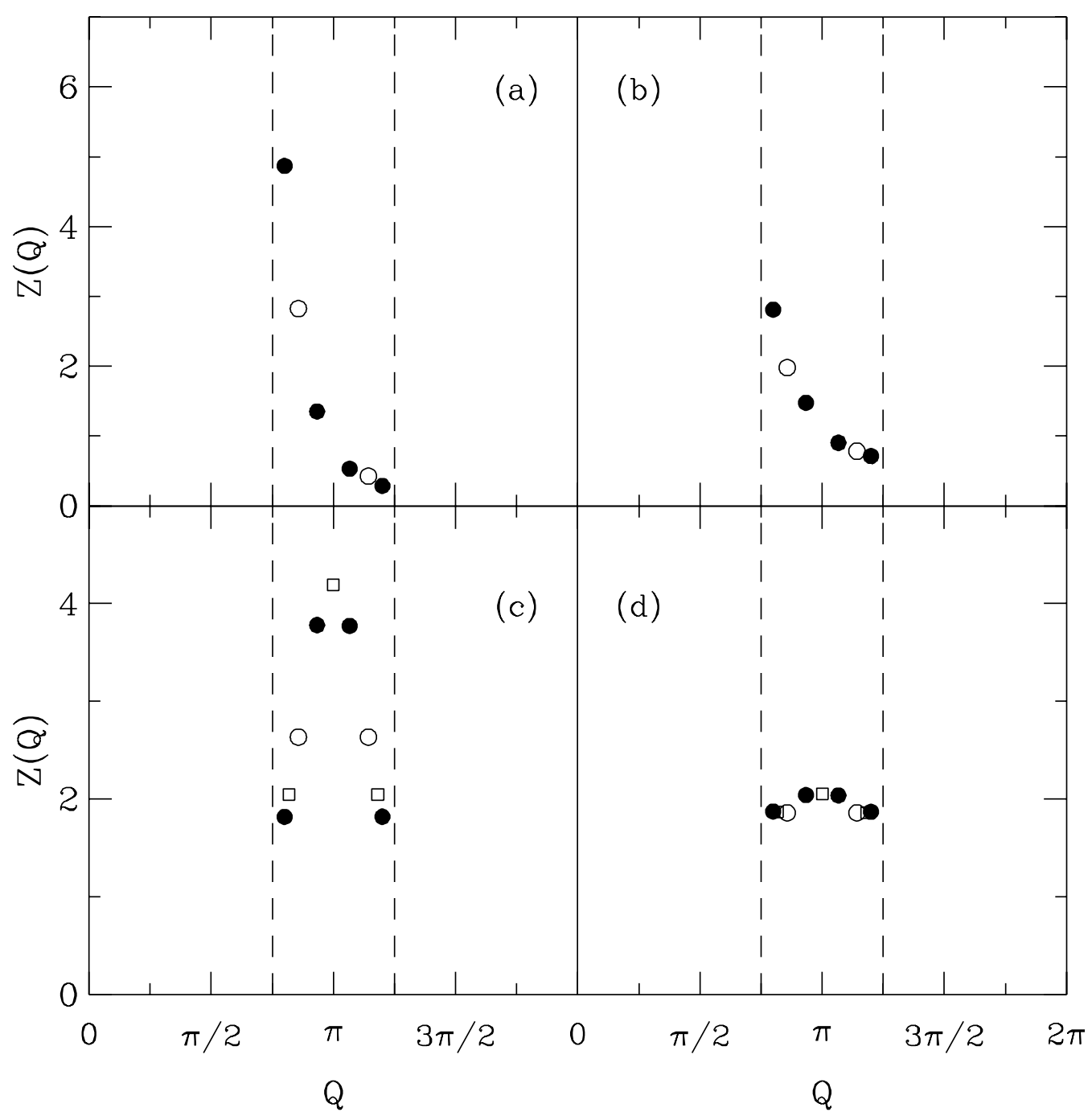

Sorella and Parola Fig. 12 


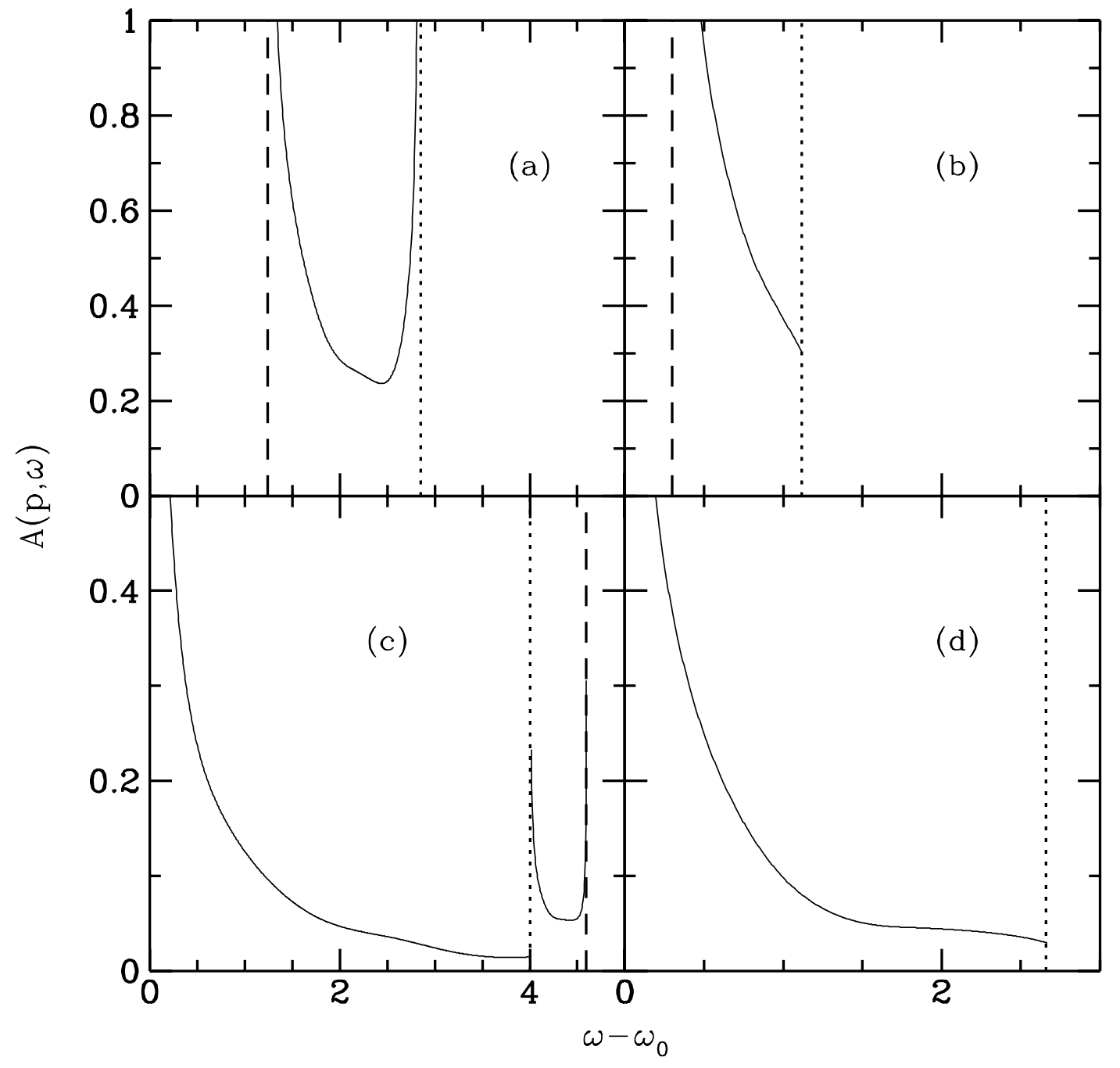

Sorella and Parola Fig. 13 